\title{
Information-Theoretic Analysis of MIMO Channel Sounding
}

Daniel S. Baum and Helmut Bölcskei

\author{
Communication Technology Laboratory \\ ETH Zurich \\ 8092 Zurich, Switzerland \\ \{dsbaum, boelcskei\}@ nari.ee.ethz.ch
}

\begin{abstract}
The large majority of commercially available multiple-input multiple-output (MIMO) radio channel measurement devices (sounders) is based on time-division multiplexed switching (TDMS) of a single transmit/receive radio frequency chain into the elements of a transmit/receive antenna array. While being cost-effective, such a solution can cause significant measurement errors due to phase noise and frequency offset in the local oscillators. In this paper, we systematically analyze the resulting errors and show that, in practice, overestimation of channel capacity by several hundred percent can occur. Overestimation is caused by phase noise (and to a lesser extent frequency offset) leading to an increase of the MIMO channel rank. Our analysis furthermore reveals that the impact of phase errors is, in general, most pronounced if the physical channel has low rank (typical for line-of-sight or poor scattering scenarios). The extreme case of a rank-1 physical channel is analyzed in detail. Finally, we present measurement results obtained from a commercially employed TDMS-based MIMO channel sounder. In the light of the findings of this paper, the results obtained through MIMO channel measurement campaigns using TDMS-based channel sounders should be interpreted with great care.
\end{abstract}

\section{Index Terms}

Wireless, channel measurements, MIMO, multiplexing, phase noise, frequency offset paper was presented in part at IEEE VTC Fall 2004, Sept. 2004, Los Angeles, CA. 


\section{INTRODUCTION}

Multiple-input multiple-output (MIMO) wireless communication promises significant improvements over existing wireless systems both in terms of spectral efficiency and link reliability. Obtaining accurate measurements of MIMO radio channels is of key importance to devising accurate MIMO radio channel models, which in turn are vital for system design, simulation, and performance analysis.

A common and widespread MIMO channel measurement device (a.k.a. sounder) design is based on time-division multiplexing with synchronous switching, or time-division multiplexed switching (TDMS) for short, of a single radio frequency (RF) chain into the individual elements of an antenna array. TDMS can be used at either the transmitter or the receiver (one-sided TDMS) or at both sides of the link (double-sided TDMS). For the latter case, which is practically the most relevant one, such an architecture is depicted in Fig. 1. TDMS constitutes a natural extension of single-input single-output (SISO) channel sounders and leads to very cost-effective solutions as only a single RF chain is required at either the transmitter or the receiver (one-sided TDMS) or at both sides of the link (double-sided TDMS). To the best of our knowledge, the large majority of commercially available MIMO channel sounders is based on the TDMS principle. A major drawback of TDMS-based sounder architectures results from temporal phase deviations between the outputs of the local oscillators (LOs) in the RF chains at transmitter and receiver being translated into the spatial domain due to switching across antenna elements. This can cause an increase of the MIMO channel rank and corresponding measurement errors, in terms of estimated MIMO channel capacity, that can be on the order of several hundred percent. It is therefore immediately clear that understanding the impact of phase errors 1 in TDMS-based sounding is of fundamental importance.

One may argue that in a wireless communication link the impact of phase fluctuations in the

\footnotetext{
${ }^{1}$ For brevity, in the remainder of this paper, we use the terminology phase errors whenever we refer to phase deviations due to phase noise, or frequency offset, or both.
} 
transmitter and/or the receiver can simply be absorbed into an effective channel consisting of the physical propagation channel combined with LO-related (and potentially other) impairments. Channel estimation at the receiver for demodulation and decoding or for precoding at the transmitter (through feedback) would then simply work on the effective channel. This point of view can certainly be sensible in a data transmission setup if the frequency dispersion caused by phase fluctuations is small compared to that induced by the physical channel. In a channel sounding setup, however, it is crucial to separate the physical propagation channel (i.e., the object to be measured) from transmitter/receiver impairments, in order to obtain measurement results that depend as little as possible on the measurement device (sounder) used. Furthermore, as already pointed out, the measurement procedure employed in TDMS-based MIMO channel sounding results in very high sensitivity of the estimated channel capacity with respect to phase errors.

Contributions: The goal of this paper is to systematically analyze the impact of phase noise and frequency offset (between transmitter and receiver LO) on estimated MIMO channel capacity when TDMS-based channel sounders are used. In particular, we show that the presence of phase errors can lead to significant overestimation of MIMO channel capacity. A sensitivity analysis reveals that, in certain cases, underestimation is possible as well, albeit typically resulting in significantly smaller errors.

We start by devising a signal model, applicable to the wide class of correlation-based (as defined in [1, Sec. III]) MIMO channel sounders and taking into account phase noise and frequency offset. We then systematically identify situations where phase errors have no (or little) impact on MIMO (ergodic and outage) capacity estimates and where they lead to significant estimation errors. As an extreme case in the latter category, we demonstrate that even moderate phase noise can turn a rank-1 physical channel (e.g., a pin-hole channel [2], [3]) into a fullrank effective channel; analytic expressions for the corresponding (ergodic and outage) capacity estimates are provided. Our analytic results are supported by measurement results obtained from 
a commercially employed TDMS-based MIMO channel sounder.

Previous related work: For linear time-varying SISO physical channels, an analysis of the systematic measurement errors incurred by correlative channel sounders (due to the time-varying nature of the physical channel) is reported in [1]. Models for the phase noise power spectral density (PSD) of a $5 \mathrm{GHz}$ and a $50 \mathrm{GHz}$ frequency synthesizer as well as an expression for the phase-noise induced reduction of the dynamic range of m-sequences can be found in [4]. A subsequent paper by the same authors [5] discusses the impact of frequency offset on direction of arrival (DOA) estimates in single-input multiple-output (SIMO) TDMS-based measurements and proposes corresponding mitigation methods. The effect of random-walk phase noise on the root mean-square (rms) error of SAGE2 -based DOA estimates is analyzed in [7].

For fully parallel MIMO channel sounders, i.e., channel sounders employing a separate RF chain for each transmit and each receive antenna element, the impact of gain imbalance in parallel RF chains and of thermal noise on the estimated capacity of a physical rank-1 MIMO channel is analyzed in [8]. The variance of an approximation of the error in the mutual information (MI) of an effective channel resulting from a deterministic MIMO channel subject to additive white complex Gaussian distributed perturbations is derived in [9]. For physical pin-hole [2], a.k.a. key-hole [3], [10], [11] (i.e., rank-1), MIMO channels in a controlled indoor environment, the impact of measurement imperfections such as thermal noise and "multi-path leakage" (i.e., multi-path components propagating between the transmitter and the receiver via paths other than through the pin-hole) on the channel eigenvalue distribution and the resulting outage capacity are analyzed numerically, based on measurements and simulations, in [11].

Organization of the paper: The remainder of this paper is organized as follows. In Section the architecture of a TDMS-based MIMO channel sounder is described and the corresponding channel and signal model are provided. In Section III, we analyze the effect of phase errors on

\footnotetext{
${ }^{2}$ Space-alternating generalized expectation maximization (EM) algorithm [6].
} 
MIMO channel statistics. The corresponding impact on estimated MI is studied in Section IV A framework for analyzing the sensitivity of the MIMO channel MI to phase errors is developed in Section $\nabla$. Section $\nabla \mathrm{I}$ is devoted to the special (but practically relevant) case of rank-1 physical channels. Measurement results performed on a typical, commercially employed TDMS-based MIMO channel sounder corroborating our analysis are presented in Section VII. We conclude in Section VIII.

Notation: $\mathbb{E}\{\cdot\}$ denotes the expectation operator. $f(t) * g(t)=\int_{\tau} f(\tau) g(t-\tau) \mathrm{d} \tau$ stands for the convolution of the functions $f(t)$ and $g(t)$. The Dirac delta function is denoted as $\delta(t)$, and $\delta_{i}=1$ for $i=0$ and 0 otherwise. The superscripts ${ }^{T},{ }^{H}$, and ${ }^{*}$ stand for transposition, conjugate transposition, and elementwise conjugation, respectively. An $m \times n$ matrix is a matrix with $m$ rows and $n$ columns. 1 and $\mathbf{0}$ denote an all-ones and all-zeros matrix, respectively, of appropriate size. If required, the size of a matrix is specified through subscripts, e.g., $\mathbf{1}_{m, n} . \mathbf{I}_{m}$ stands for the $m \times m$ identity matrix. $\mathbf{A} \circ \mathbf{B}$ and $\mathbf{A} \otimes \mathbf{B}$ denote the Hadamard (pointwise) product and the Kronecker product, respectively, of the matrices $\mathbf{A}$ and $\mathbf{B}$, and $f^{\circ}(\mathbf{A})$ stands for the matrix resulting from entry-wise application of the function $f(\cdot)$ to $\mathbf{A} .\|\mathbf{A}\|_{\mathrm{F}}$ is the Frobenius norm of $\mathbf{A}$ and $\operatorname{Tr}(\mathbf{A})$ is the trace of $\mathbf{A} \cdot \operatorname{diag}(\mathbf{x})$ denotes the diagonal matrix with the elements of the vector $\mathbf{x}$ on its main diagonal, and $\operatorname{dg}(\mathbf{A})=\mathbf{A} \circ \mathbf{I}$ zeros out all but the diagonal elements of $\mathbf{A}$. The element in the $m$ th row and $n$th column of $\mathbf{A}$ is denoted as $[\mathbf{A}]_{m, n} . r(\mathbf{A})$ and $\lambda_{i}(\mathbf{A})$ stand for the rank and the $i$ th eigenvalue of $\mathbf{A}$, respectively. Unless explicitly stated otherwise, eigenvalues are sorted in decreasing order, i.e., $\lambda_{1}(\mathbf{A}) \geq \lambda_{2}(\mathbf{A}) \geq \cdots \geq \lambda_{n}(\mathbf{A})$. For an $m \times n$ matrix $\mathbf{A}=\left[\begin{array}{llll}\mathbf{a}_{1} & \mathbf{a}_{2} & \cdots & \mathbf{a}_{n}\end{array}\right]$ with columns $\mathbf{a}_{i}$, we define the $m n \times 1$ vector $\operatorname{vec}(\mathbf{A})=\left[\begin{array}{llll}\mathbf{a}_{1}^{T} & \mathbf{a}_{2}^{T} & \cdots & \mathbf{a}_{n}^{T}\end{array}\right]^{T}$. The commutation matrix $\mathbf{K}_{(m, n)}$ [12, Sec. 3.7] is a permutation matrix of size $m n \times m n$ uniquely defined through

$$
\mathbf{K}_{(m, n)} \operatorname{vec}(\mathbf{A})=\operatorname{vec}\left(\mathbf{A}^{T}\right)
$$


where $\mathbf{A}$ is an $m \times n$ matrix. For brevity, we $\operatorname{define} \operatorname{divec}(\mathbf{A})=\operatorname{diag}(\operatorname{vec}(\mathbf{A}))$. For two random variables (RVs) $X$ and $Y, X \stackrel{\mathrm{d}}{=} Y$ and $X \stackrel{\mathrm{d}}{\approx} Y$ stands for equivalence and approximate equivalence in distribution, respectively. For a $\mathrm{RV} X$, the probability density function (pdf) and cumulative distribution function (cdf) are denoted by $\mathrm{p}_{X}(x)$ and $\mathrm{F}_{X}(x)$, respectively, and the moment-generating function (MGF) is defined as $M_{X}(s)=\mathbb{E}\{\exp (s X)\}$. The variance of a $\mathrm{RV} X$ is denoted as $\operatorname{Var}\{X\}$. The covariance matrix of a complex random matrix $\mathbf{X}$ is defined as $\operatorname{Cov}\{\mathbf{X}\}=\mathbb{E}\left\{(\operatorname{vec}(\mathbf{X})-\mathbb{E}\{\operatorname{vec}(\mathbf{X})\})(\operatorname{vec}(\mathbf{X})-\mathbb{E}\{\operatorname{vec}(\mathbf{X})\})^{H}\right\}$ and the corresponding matrix of correlation coefficients is the matrix with entries $[\operatorname{Corr}\{\mathbf{X}\}]_{m, n}=$ $[\operatorname{Cov}\{\mathbf{X}\}]_{m, n} /\left([\operatorname{Cov}\{\mathbf{X}\}]_{m, m}[\operatorname{Cov}\{\mathbf{X}\}]_{n, n}\right)^{1 / 2}$. The pseudo-covariance matrix [13] of a complex random matrix $\mathbf{X}$ is defined as $\operatorname{Cov}_{\mathrm{p}}\{\mathbf{X}\}=\mathbb{E}\left\{(\operatorname{vec}(\mathbf{X})-\mathbb{E}\{\operatorname{vec}(\mathbf{X})\})(\operatorname{vec}(\mathbf{X})-\mathbb{E}\{\operatorname{vec}(\mathbf{X})\})^{T}\right\}$. For the integers $a$ and $b, a \operatorname{div} b=\lfloor a / b\rfloor$ denotes the integer division of $a$ by $b$, and $a \bmod b$ stands for the remainder, on division of $a$ by $b$. For a complex scalar $z \in \mathbb{C}$, the function $\arg (z)$ returns the argument (angle) of $z$ in the interval $[0,2 \pi)$, and $\operatorname{Re}(z)$ and $\operatorname{Im}(z)$ stand for the real and imaginary part of $z$, respectively. All logarithms are to the base 2 unless explicitly stated otherwise. $|\mathfrak{I}|$ denotes the length of an interval $\mathfrak{I}$. Throughout the paper, the number of transmit and receive antenna elements in a MIMO channel is denoted as $M_{\mathrm{T}}$ and $M_{\mathrm{R}}$, respectively, and we will refer to antenna elements simply as antennas.

A real Gaussian random vector is defined as a vector with jointly Gaussian (JG) elements and denoted by $\mathcal{N}(\mathbf{m}, \mathbf{C})$, where $\mathbf{m}$ is the mean and $\mathbf{C}$ is the covariance matrix. A complex Gaussian random vector is defined as a vector with JG real and imaginary parts. A complex random vector will be called proper if its pseudo-covariance matrix vanishes. $\mathcal{C N}(\mathbf{m}, \mathbf{C})$ stands for a proper complex Gaussian random vector with mean $\mathbf{m}$ and covariance matrix $\mathbf{C}$. The complex random vectors $\mathbf{x}$ and $\mathbf{y}$ will be called jointly proper if the composite random vector having $\mathbf{x}$ and $\mathbf{y}$ as subvectors is proper.

For a chi-square distributed RV with $n$ degrees of freedom and variance $2 n \sigma^{4}$ we write $\chi_{n, \sigma^{2}}^{2}$, 
where $\chi_{n, \sigma^{2}}^{2} \stackrel{\mathrm{d}}{=}\|\mathbf{x}\|^{2}$ with $\mathbf{x} \stackrel{\mathrm{d}}{=} \mathcal{N}\left(\mathbf{0}, \sigma^{2} \mathbf{I}_{n}\right) .\left[\begin{array}{llll}x_{1} & x_{2} & \cdots & x_{n}\end{array}\right] \stackrel{\mathrm{d}}{=} D_{n}\left(a_{1}, a_{2}, \ldots, a_{n}\right), x_{i} \geq 0$ $(i=1,2, \ldots, n)$ denotes a Dirichlet distributed random vector with parameters $a_{1}, a_{2}, \ldots, a_{n}$. The corresponding subvector $\mathbf{x}=\left[\begin{array}{llll}x_{1} & x_{2} & \cdots & x_{n-1}\end{array}\right]$ satisfies $\mathbf{x} \stackrel{\mathrm{d}}{=} D_{n-1}\left(a_{1}, a_{2}, \ldots, a_{n-1} ; a_{n}\right)$ and has joint pdf [14, Th. 1.2]

$$
\mathrm{p}_{D_{n-1}\left(a_{1}, a_{2}, \ldots, a_{n-1} ; a_{n}\right)}(\mathbf{x})=\frac{\Gamma(a)}{\prod_{i=1}^{n} \Gamma\left(a_{i}\right)}\left(\prod_{i=1}^{n-1} x_{i}^{a_{i}-1}\right)\left(1-\sum_{i=1}^{n-1} x_{i}\right)^{a_{n}-1}
$$

where $a=\sum_{i=1}^{n} a_{i}$ and $\Gamma(z)$ is the Gamma function [15, Sec. 6.1]. We denote the beta distribution with parameters $a$ and $b$ as $\beta(a, b)$ with pdf given by

$$
\mathrm{p}_{\beta(a, b)}(x)=\frac{\Gamma(a+b)}{\Gamma(a) \Gamma(b)}(1-x)^{b-1} x^{a-1}, \quad a>0, b>0, x \geq 0
$$

The digamma function $\Psi(z)$ is defined as [15, Eq. 6.3.1, Eq. 6.3.16]

$$
\begin{aligned}
& \Psi(z)=\frac{\mathrm{d}}{\mathrm{d} z} \log _{e} \Gamma(z)=\frac{\Gamma^{\prime}(z)}{\Gamma(z)}=\psi_{0}(z), \quad z \in \mathbb{C} \\
& \Psi(z)=-\gamma+\sum_{n=1}^{\infty} \frac{z}{n(n+z-1)}, \quad z \neq 0,-1,-2, \ldots
\end{aligned}
$$

where $\gamma \approx 0.5772$ is Euler's constant, and $\psi_{n}(z)$ is the polygamma function [15, Eq. 6.4.1], defined as the $n$th derivative of $\Psi(z)$. We will also need the following representation of the digamma function at positive integer multiples of $1 / 2$ given by [15, Eq. 6.3.2, Eq. 6.3.4]

$$
\Psi(k)=-\gamma+\sum_{n=1}^{k-1} \frac{1}{n}, \quad \Psi\left(k-\frac{1}{2}\right)=-\gamma-2 \log _{e}(2)+\sum_{n=1}^{k-1} \frac{2}{2 n-1}, \quad k \in \mathbb{N}, k \geq 1
$$

Finally, we note that the first derivative of the digamma function can be written as an infinite series as [15, Eq. 6.4.10]

$$
\Psi^{\prime}(z)=\psi_{1}(z)=\frac{\mathrm{d}^{2}}{\mathrm{~d} z^{2}} \log _{e} \Gamma(z)=\sum_{n=1}^{\infty} \frac{1}{(n+z-1)^{2}}, \quad z \neq 0,-1,-2, \ldots
$$




\section{MiMO Channel Sounding Based On Time-Division MultipleXed Switching}

In this section, we describe the system architecture of a correlation-based MIMO channel sounder employing TDMS and we present the corresponding signal model, taking into account the presence of phase errors.

\section{A. Channel Sounder Architecture}

The basic architecture of a TDMS-based MIMO channel sounder is depicted in Fig. 1. A (possibly complex) sounding signal $x(t)$ is generated in baseband and modulated to the propagation channel center frequency. At the receiver, bandpass filtering (to remove out-of-band thermal noise) and downconversion to baseband, resulting in the signal $u(t)$, is followed by extraction of the MIMO channel estimate. Both the transmitter and the receiver employ a multiplexing unit, which steps a single RF chain through all transmit/receive antennas sequentially in time following a prescribed switching pattern. Clocks at transmitter and receiver serve as reference for the analog-to-digital converter (ADC) and digital-to-analog converter (DAC) sampling rates, antenna multiplexing timing, and RF mixing (i.e., LO) frequencies.

Another frequently used MIMO channel sounder setup employs a single antenna (either at the transmitter or the receiver or at both sides of the link), which is physically moved (in an automated fashion) to form a "virtual" antenna array. This setup also fits into the framework described in the paper. The impact of phase errors on MIMO channel capacity estimates in such a timedivision-based "virtual" antenna array sounder architecture will, in general, be significantly more pronounced than in the TDMS-based case where a single RF chain is switched electronically into different physical antenna elements. This is because in the "virtual" antenna array case, the time that passes when moving the single antenna from a given physical position to the next one is much longer than the time it takes to switch electronically between different antenna elements. 


\section{B. SISO Signal Model}

We start by presenting the signal model for a correlative SISO channel sounder in the presence of phase errors. This constitutes the basis for the MIMO signal model in TDMS-based sounders introduced in Section $\amalg-\mathrm{C}$.

Apart from frequency offset, a difference between the reference clocks at transmitter and receiver also causes a difference in the corresponding baseband sampling rates. The effect of this sampling rate offset, however, can be neglected compared to the frequency offset incurred by up- and down-conversion with respect to the LO frequencies, which are significantly higher than the sampling rates. The signal model presented below will therefore not account for sampling rate offset.

1) Correlation-based sounding: The baseband sounding signal is given by $x(t)=\sum_{k} s(t-$ $t_{k}$ ) where $s(t)$, supported on an inverval $\mathfrak{I}_{\mathrm{T}}$, denotes the convolution of the (time-continuous) transmit sounding sequence 3 with the impulse response of the transmit frontend filter. Further, $t_{k}$ is the SISO snapshot (measurement) time instant, referred to as snapshot time in the following, and $k$ denotes the SISO snapshot index. Because the sounder can have a measurement duty cycle less than one, the time intervals $\left[t_{k-1}, t_{k}\right]$ generally contain measurement time and void time. In the following, we will refer to the quantities $t_{k}-t_{k-1}$ as the SISO snapshot time distances. After bandpass filtering and downconversion, the receiver applies a linear time-invariant (LTI) filter with impulse response $r(t)$, supported on an inverval $\mathfrak{I}_{\mathrm{R}}$, and consisting of the receive sounding sequence convolved with the receive frontend filter. The signals $s(t)$ and $r(t)$ are chosen such that the function

$$
c(t)=s(t) * r(t)
$$

\footnotetext{
${ }^{3}$ The sounding sequence is a weighted chip-spaced Dirac train with the weights determined by the time-discrete sounding signal, e.g. a binary phase-shift keying (BPSK)-modulated m-sequence.
} 
is peaky around $t=0$, where it takes on its maximum value

$$
c(0)=\int_{\mathfrak{I}_{\mathrm{TR}}} s(t) r(-t) \mathrm{d} t=1
$$

with $\mathfrak{I}_{\mathrm{TR}}=\mathfrak{I}_{\mathrm{T}} \cap\left(-\mathfrak{I}_{\mathrm{R}}\right)$. In the following, we will be interested in the behavior of the function $c(t)$ in an interval $\mathfrak{I}_{\mathrm{c}}$ around $t=0$. The sequence signal-to-noise ratio (SNR) quantifies the peakiness of $c(t)$ (in an interval $\mathfrak{I}_{\mathrm{c}}$ ).

In the sequel, we denote channel sounders satisfying the conditions described in the previous paragraph as correlation(-based) sounders. In the literature, the term correlation sounder is usually reserved for a more specific setup where $r(t)=s^{*}(-t)$ with $s(t)$ resulting from a BPSK modulated pseudo-(random) noise sequence (PNS) (typically an m-sequence). The widely used class of channel sounders employing chirp or multisine sounding sequences [1], [16]-[19] satisfies the conditions stated in the previous paragraph and hence fits into our framework.

2) Modeling phase noise and frequency offset: The LOs in the transmitter and the receiver generate signals that have the analytic signal representations

$$
o_{\mathrm{T}}(t)=e^{j\left(2 \pi f_{\mathrm{T}} t+\varphi_{\mathrm{T}}(t)\right)} \quad \text { and } \quad o_{\mathrm{R}}(t)=e^{j\left(2 \pi f_{\mathrm{R}} t+\varphi_{\mathrm{R}}(t)\right)}
$$

respectively, where $f_{\mathrm{T}}$ and $f_{\mathrm{R}}$ are the corresponding desired LO frequencies, and $\varphi_{\mathrm{T}}(t)$ and $\varphi_{\mathrm{R}}(t)$ represent the corresponding additive LO phase. In the remainder of this paper, we assume that $\varphi_{\mathrm{T}}(t)$ and $\varphi_{\mathrm{R}}(t)$ are real-valued zero-mean wide-sense stationary (WSS) Gaussian random processes. We shall next justify the modeling assumptions made in this paragraph.

The characterization of phase and frequency instabilities in precision frequency sources has been a major area of research for many years [20]-[26]. In the ensuing discussion, we distinguish between the directly observable random process $o(t)=\exp (j(2 \pi f t+\varphi(t)))$ and the underlying phase process $\varphi(t)$. There are two fundamentally different models for $\varphi(t)$ motivated by the corresponding different methods of frequency generation, namely free-running and closed-loop. 
In the case of free-running oscillators, the phase process is often modeled as a continuous-time Wiener (a.k.a. Brownian motion) process, which is Gaussian and nonstationary, with autocovariance function $\mathbb{E}\{\varphi(t) \varphi(t+\tau)\} \propto \min (t, t+\tau)$ [23, Corollary 7.1]. It is important to note that even though the phase process is nonstationary and has a variance that grows without bounds over time, the corresponding observable process is stationary with finite power and has an approximately Lorentzian (i.e., one-pole lowpass) PSD function around the first harmonic [23, Eq. (41)]. In the case of a closed-loop (a.k.a. phase-feedback) system, such as a phaselocked loop (PLL), in the locked and steady state, the phase difference between its input and output signal can be modeled as a Gaussian WSS process with bounded variance. In practice, frequency generation is often performed by locking one or more PLLs to the output of a freerunning oscillator, which results in an overall phase process $\varphi(t)$ that is the sum of a Brownian motion process and an asymptotically (in time) Gaussian WSS process [25]. Generally speaking, the components in $\varphi(t)$ corresponding to the free-running oscillator and to the PLLs dominate the long-term and the short-term phase process behavior, respectively. In TDMS-based MIMO channel sounding, the time-scale of interest, determined by the duration of one MIMO snapshot (i.e., the duration it takes to measure all scalar subchannels of the MIMO channel), is such that the behavior of the phase process is typically dominated by the component due to the PLL(s). The nonstationary (Brownian motion) component in $\varphi(t)$ can therefore be neglected. We shall support this statement in Section VII through measurements 4 performed on a commercially employed TDMS-based MIMO channel sounder.

The absolute frequencies generated by the independent clock sources at transmitter and receiver can differ significantly, which is accounted for by allowing a nonzero frequency offset $\Delta \omega=$ $2 \pi\left(f_{\mathrm{T}}-f_{\mathrm{R}}\right)$. The resulting overall model, specified below, captures all relevant effects in a

\footnotetext{
${ }^{4}$ While we did not observe the Brownian motion component within the duration of a MIMO snapshot in our measurements, we do acknowledge that it may occur for the channel sounder under consideration if the MIMO snapshot duration is sufficiently long.
} 
simple and mathematically tractable manner.

3) SISO signal model: We are now ready to state the SISO signal model including the effects of phase errors. Throughout the paper, we shall work in complex baseband. We assume that the (time-varying) physical channel to be measured is LTI during each snapshot interval and can change from snapshot to snapshot. The impulse response during the $k$ th snapshot interval $t_{k}+\mathfrak{I}_{\mathrm{T}}(k \in \mathbb{Z})$ is given by

$$
h_{k}(t)=\sum_{l} h_{k}^{(l)} \delta\left(t-\tau_{k}^{(l)}\right), \quad \tau_{k}^{(m)} \neq \tau_{k}^{(n)} \forall m \neq n
$$

and the individual snapshot intervals do not overlap.

The overall signal model (in complex baseband notation) for the $k$ th snapshot is hence given as

$$
u_{k}(t)=r(t) *\left(o_{\mathrm{R}, \mathrm{bb}}^{*}(t)\left(h_{k}(t) *\left(o_{\mathrm{T}, \mathrm{bb}}(t) s\left(t-t_{k}\right)\right)\right)+n^{\prime}(t)\right)
$$

where we set $o_{\mathrm{T}, \mathrm{bb}}(t)=o_{\mathrm{T}}(t) \exp \left(-j 2 \pi f_{\mathrm{T}} t\right)=\exp \left(j \varphi_{\mathrm{T}}(t)\right)$ and $o_{\mathrm{R}, \mathrm{bb}}(t)=o_{\mathrm{R}}(t) \exp \left(-j 2 \pi f_{\mathrm{T}} t\right)$ $=\exp \left(-j\left(\Delta \omega t-\varphi_{\mathrm{R}}(t)\right)\right)$, and $n^{\prime}(t)$ represents thermal noise at the receiver input. Straightforward manipulations yield

$$
u_{k}(t)=\sum_{l} h_{k}^{(l)} r(t) *(\underbrace{e^{j \Delta \omega t} e^{-j \varphi_{\mathrm{R}}(t)} e^{j \varphi_{\mathrm{T}}\left(t-\tau_{k}^{(l)}\right)}}_{\theta_{k, l}\left(t-t_{k}-\tau_{k}^{(l)}\right)} s\left(t-t_{k}-\tau_{k}^{(l)}\right))+n(t)
$$

where $n(t)=r(t) * n^{\prime}(t)$. In the absence of phase errors, i.e., $\varphi_{\mathrm{T}}(t)=\varphi_{\mathrm{R}}(t)=\Delta \omega=0$, we have

$$
u_{k}(t)=\sum_{l} h_{k}^{(l)} c\left(t-t_{k}-\tau_{k}^{(l)}\right)+n(t)
$$

Now, if the interval $\mathfrak{I}_{\mathrm{c}}$ is such that $\tau_{k}^{(l)} \in \mathfrak{I}_{\mathrm{c}} \forall k, l$, the peakiness of $c(t)=s(t) * r(t)$ (within $\mathfrak{I}_{\mathrm{c}}$ ) implies that the channel coefficients $h_{k}^{(l)}$ can be retrieved by sampling $u_{k}(t)$ at the time instants $t_{k}+\tau_{k}^{(l)}$. The measurement SNR in extracting the coefficients $h_{k}^{(l)}$ from $u_{k}(t)$ is clearly limited, 
among other factors, by the sequence SNR. From (7), we can see that in the presence of phase errors, one has to deal with the quantity

$$
c_{k, l}^{\prime}(t)=\left(\theta_{k, l}(t) s(t)\right) * r(t)
$$

instead of $c(t)$ as in (8). Phase errors, accounted for by the term $\theta_{k, l}(t)$, therefore can lead to a shifting of the peak of $c(t)$ (which is at $t=0$ in the phase-error free case) and to a degradation of the correlation properties, or equivalently, the peakiness of $c(t)$ as quantified by the sequence SNR. We shall next show that, under quite general conditions, we can write

$$
c_{k, l}^{\prime}(t)=c_{k, l} p_{k, l}(t)
$$

where $p_{k, l}(0)=1 \forall k, l$, and the $p_{k, l}(t)$ are functions that have their peaks at $t=0$. As a consequence of (9), we then obtain

$$
u_{k}(t)=\sum_{l} h_{k}^{(l)} c_{k, l} p_{k, l}\left(t-t_{k}-\tau_{k}^{(l)}\right)+n(t)
$$

which implies that the channel coefficients in the presence of phase errors are given by $h_{k}^{(l)} c_{k, l}$ and can still be obtained by sampling $u_{k}(t)$ at the time instants $t_{k}+\tau_{k}^{(l)}$. Moreover, the functions $p_{k, l}(t)$ will be less peaky than $c(t)$ (within the interval $\mathfrak{I}_{\mathrm{c}}$ ), which results in a reduction of the sequence SNR and hence the measurement SNR in terms of extracting the channel coefficients by sampling the function $u_{k}(t)$.

A general but restrictive condition for the $p_{k, l}(t), \forall k, l$ to have their peaks at $t=0$ is that $\theta_{k, l}(t), \forall k, l$ be narrowband relative to $s(t)$ which would imply $c_{k, l}^{\prime}(t) \propto c(t)$. This "slow phase noise" condition, however, is in practice hardly satisfied. A more systematic approach to assessing the degradation of sequence SNR due to phase errors consists of decomposing $\theta_{k, l}(t)$ into the 
harmonics $\exp \left(j \omega_{d} t\right)$ and evaluating the behavior of the quantity

$$
\left(s(t) e^{j \omega_{d} t}\right) * r(t)=\int_{-\infty}^{\infty} s(-\xi) r(t+\xi) e^{-j \omega_{d} \xi} \mathrm{d} \xi
$$

which for $r(t)=s^{*}(-t)$ becomes Woodward's correlation function [27, Ch. 7, Eq. (17)]

$$
A_{s^{-}}\left(t, \omega_{d}\right)=\int_{-\infty}^{\infty} s(-\xi) s^{*}(-\xi-t) e^{-j \omega_{d} \xi} \mathrm{d} \xi
$$

where $s^{-}$stands for $s(-t)$. In the remainder of this paragraph, we restrict ourselves to $r(t)=$ $s^{*}(-t)$, for simplicity. In the general case Woodward's correlation function is replaced by the cross-correlation function between $s(t)$ and $r(t)$. Denoting the Fourier transform of $\theta_{k, l}(t)$ by $\Theta_{k, l}(j \omega)=\int_{-\infty}^{\infty} \theta_{k, l}(t) e^{-j \omega t} d t$, it follows that

$$
c_{k, l}^{\prime}(t)=\frac{1}{2 \pi} \int_{-\infty}^{\infty} \Theta_{k, l}(j \omega) A_{s^{-}}(t, \omega) \mathrm{d} \omega .
$$

Analyzing the quantity $\mathbb{E}\left\{\left|c_{k, l}^{\prime}(t)\right|^{2}\right\}$, it can be verified that for m-sequences (used, e.g., in the channel sounder analyzed in Section VII $A_{s^{-}}(t, \omega)$ is such that, under quite general conditions on the phase noise PSD, peak-shifting does not occur, i.e., $c_{k, l}^{\prime}(t)$ has its peak at $t=0, \forall k, l$, and hence (9) is satisfied. However, for sounders employing, for example, chirp sequences peakshifting does occur.

We shall next show that, under assumptions validated by our measurements in Section VII, (9) can be simplified further in the sense that the $c_{k, l}$ do not depend on $l$. Straightforward manipulations reveal that

$$
c_{k, l}=e^{j \Delta \omega\left(t_{k}+\tau_{k}^{(l)}\right)} \int_{\mathfrak{I}_{\mathrm{TR}}} e^{j \Delta \omega \xi} e^{-j \varphi_{\mathrm{R}}\left(t_{k}+\tau_{k}^{(l)}+\xi\right)} e^{j \varphi_{\mathrm{T}}\left(t_{k}+\xi\right)} w(\xi) \mathrm{d} \xi
$$

where $w(t)=s(t) r(-t)$ is a window function. For BPSK modulated m-sequences with rectangular chip pulses (used in the sounder analyzed in Section VII) and $r(t)=s^{*}(-t)$, for example, we have $w(t)=$ const. for $t \in \mathfrak{I}_{\mathrm{TR}}$. Next, assuming that 
i) $\varphi_{\mathrm{R}}(t), \varphi_{\mathrm{T}}(t)$, and $\Delta \omega t$ do not change appreciably during an interval of length $\left|\mathfrak{I}_{\mathrm{TR}}\right|$, and thus, recalling the normalization of $w(t)$ according to (5),

$$
\left|\int_{\mathfrak{I}_{\mathrm{TR}}} e^{j \Delta \omega \xi} e^{-j \varphi_{\mathrm{R}}\left(t_{k}+\tau_{k}^{(l)}+\xi\right)} e^{j \varphi_{\mathrm{T}}\left(t_{k}+\xi\right)} w(\xi) \mathrm{d} \xi\right| \approx 1
$$

ii) the delay spread is small enough for

$$
\Delta \omega \tau_{k}^{(l)} \approx 0 \quad \text { and } \quad \varphi_{\mathrm{R}}\left(t_{k}+\tau_{k}^{(l)}+\xi\right) \approx \varphi_{\mathrm{R}}\left(t_{k}+\xi\right), \quad \forall k, l
$$

to hold,

and further assuming without loss of generality (w.l.o.g.) that the interval $\mathfrak{I}_{\mathrm{TR}}$ is symmetric around $t=0$, we obtain

$$
c_{k, l} \approx e^{j \Delta \omega t_{k}} e^{j \varphi_{k}}
$$

which, obviously, does not depend on $l$. Here, $\varphi_{k}$ is a real-valued WSS Gaussian random process with zero-mean and variance $\sigma_{\varphi}^{2}$. We note that formally the condition that $\varphi_{\mathrm{R}}(t)$ does not change appreciably during an interval of length $\left|\mathfrak{I}_{\mathrm{TR}}\right|$ implies the second condition in (13) as the delay spread is significantly smaller than $\left|\mathfrak{I}_{\mathrm{TR}}\right|$. We decided, however, to state the second condition in (13) separately in order to stress that it is more critical that the approximation error in the second condition in (13) be small. Inserting (14) into (10) finally yields

$$
u_{k}(t)=\sum_{l} h_{k}^{(l)} e^{j \Delta \omega t_{k}} e^{j \varphi_{k}} p_{k, l}\left(t-t_{k}-\tau_{k}^{(l)}\right)+n_{k}
$$

which implies that the impact of phase noise and frequency offset is to modulate the (potentially frequency-selective) physical channel process according to

$$
\hat{h}_{k}^{(l)}=h_{k}^{(l)} e^{j \Delta \omega t_{k}} e^{j \varphi_{k}}, \quad \forall l .
$$


For flat-fading physical channels, considered in the remainder of the paper, we simply get

$$
\hat{h}_{k}=h_{k} e^{j \mu_{k}} e^{j \varphi_{k}}
$$

where we set $\mu_{k}=\Delta \omega t_{k}$. We conclude by noting that even though the model (16) is standard and well-known in the literature, we decided to provide a detailed derivation in order to exhibit the underlying key assumptions. In particular, as already mentioned, these assumptions will be validated in our measurements in Section VII.

In practice, processing on the receive side is often implemented through periodic convolution rather than linear convolution, as described above. The corresponding class of sounders will be called circular convolution-based in the following and entails extending $s(t)$ and $r(t)$ periodically to result in the $\left|\mathfrak{I}_{\mathrm{P}}\right|$-periodic signals $\tilde{s}(t)$ and $\tilde{r}(t)$, where $\left|\mathfrak{I}_{\mathrm{P}}\right|$ is given by the length of the sounding (transmit and receive) sequence. Consequently, $\mathfrak{I}_{\mathrm{P}}$ denotes one period of $\tilde{s}(t)$ and $\tilde{r}(t)$. The signals $u_{k}(t)$, obtained by convolving, for each $l$, with $\tilde{r}\left(t-\tau_{k}^{(l)}\right)$ rather than $r(t)$, are then sampled at the time instants $t_{k}$. As compared to the case of linear convolution, this leads to a slightly modified expression for the coefficients $c_{k, l}$ given by

$$
c_{k, l}=e^{j \Delta \omega t_{k}} \int_{\mathfrak{I}_{\mathrm{P}}} e^{j \Delta \omega \xi} e^{-j \varphi_{\mathrm{R}}\left(t_{k}+\xi\right)} e^{j \varphi_{\mathrm{T}}\left(t_{k}-\tau_{k}^{(l)}+\xi\right)} \widetilde{w}\left(\xi-\tau_{k}^{(l)}\right) \mathrm{d} \xi
$$

with $\widetilde{w}(t)=\tilde{s}(t) \tilde{r}(-t)$. Now, in order to arrive at the effective input-output relation (16), we need to slightly revise the assumptions made in the case of a linear convolution-based system. Instead of Condition i) leading to (12), we need to assume that $\varphi_{\mathrm{R}}(t), \varphi_{\mathrm{T}}(t)$, and $\Delta \omega t$ do not change appreciably during an interval of length $\left|\mathfrak{I}_{\mathrm{P}}\right|$ and thus

$$
\left|\int_{\widetilde{I}_{\mathrm{P}}} e^{j \Delta \omega \xi} e^{-j \varphi_{\mathrm{R}}\left(t_{k}+\xi\right)} e^{j \varphi_{\mathrm{T}}\left(t_{k}-\tau_{k}^{(l)}+\xi\right)} \widetilde{w}\left(\xi-\tau_{k}^{(l)}\right) \mathrm{d} \xi\right| \approx 1 .
$$

Instead of the second condition in (13), we need to assume that the delay spread is small enough 
for

$$
\varphi_{\mathrm{T}}\left(t_{k}-\tau_{k}^{(l)}+\xi\right) \approx \varphi_{\mathrm{T}}\left(t_{k}+\xi\right), \quad \forall k, l
$$

to hold. The condition $\Delta \omega \tau_{k}^{(l)} \approx 0$ is not needed. Circular convolution-based sounders have the advantage of reducing the required per SISO snapshot recording interval length $\left[5\right.$ from $\left|\mathfrak{I}_{\mathrm{TR}}\right|+\left|\mathfrak{I}_{c}\right|$ to $\left|\mathfrak{I}_{\mathrm{P}}\right|$. The inequality $\left|\mathfrak{I}_{\mathrm{TR}}\right|+\left|\mathfrak{I}_{c}\right|>\left|\mathfrak{I}_{\mathrm{P}}\right|$ is obtained by noting that in the case of circular convolution $\left|\mathfrak{I}_{\mathrm{TR}}\right|+\left|\mathfrak{I}_{c}\right|=\left|\mathfrak{I}_{\mathrm{T}}\right|+\left|\mathfrak{I}_{c}\right| \geq\left|\mathfrak{I}_{\mathrm{P}}\right|+\left|\mathfrak{I}_{c}\right|$, where we used $r(t)=s^{*}(-t)$ and the fact that the length of $\mathfrak{I}_{\mathrm{T}}$ is given by the length of the transmit sounding sequence plus the length of the transmit frontend filter impulse response. We close the discussion by noting that the channel sounder investigated in Section $\nabla I I$ is a circular convolution-based sounder.

\section{MIMO Signal Model}

The basic principle of TDMS-based MIMO channel sounding is to sequentially measure the $M_{\mathrm{T}} M_{\mathrm{R}}$ scalar subchannels of the MIMO channel. Since the individual SISO subchannels (note that we continue to use the term SISO snapshot to refer to the measurement of a subchannel of the MIMO matrix) are band-limited stochastic processes (due to finite Doppler spread), it is not necessary to assume that the subchannels are static during the entire MIMO measurement period. Rather, it suffices to choose the sampling rate in compliance with the sampling theorem and to properly align the measurements in time [28]. In the remainder of the paper, we assume that this alignment has already been performed.

Denoting the effective (i.e, the physical channel including the effect of phase errors) scalar subchannel between the $n$th $\left(n=1,2, \ldots, M_{\mathrm{T}}\right)$ transmit and the $m$ th $\left(m=1,2, \ldots, M_{\mathrm{R}}\right)$ receive antenna as $\hat{h}_{m, n}=h_{m, n} \exp \left(j\left(\mu_{m, n}+\varphi_{m, n}\right)\right)$, the corresponding effective MIMO channel matrix

\footnotetext{
${ }^{5}$ The signal recorded is the signal at the output of the receive frontend filter.
} 
can be expressed as

$$
\widehat{\mathbf{H}}=\mathbf{H} \circ \underbrace{\exp ^{\circ}(j(\mathbf{M}+\mathbf{\Phi}))}_{\boldsymbol{\Theta}}
$$

where $[\mathbf{M}]_{m, n}=\mu_{m, n}$ and $[\boldsymbol{\Phi}]_{m, n}=\varphi_{m, n}$. What we would like to measure is the physical channel matrix H. However, due to phase errors, the sounder has access to the effective channel matrix $\widehat{\mathbf{H}}$ only. The entries in $\mathbf{M}$ and $\boldsymbol{\Phi}$ depend on the switching pattern, i.e., the order in which the individual scalar subchannels are measured, and the SISO snapshot time distances. In the following, we denote a switching pattern as an ordered sequence of pairs $\left(\left(m_{1}, n_{1}\right),\left(m_{2}, n_{2}\right), \ldots,\left(m_{M_{\mathrm{T}} M_{\mathrm{R}}}, n_{M_{\mathrm{T}} M_{\mathrm{R}}}\right)\right)$ where $\left(m_{k}, n_{k}\right)$ means that the scalar subchannel $h_{m_{k}, n_{k}}$ is being measured at time $t_{k}$. Let us consider a simple example with $M_{\mathrm{T}}=M_{\mathrm{R}}=2$. The switching pattern $((1,1),(2,1),(2,2),(1,2))$ leads to

$$
\boldsymbol{\Theta}_{1}=\left[\begin{array}{cc}
e^{j\left(\mu_{1}+\varphi_{1}\right)} & e^{j\left(\mu_{4}+\varphi_{4}\right)} \\
e^{j\left(\mu_{2}+\varphi_{2}\right)} & e^{j\left(\mu_{3}+\varphi_{3}\right)}
\end{array}\right]
$$

whereas the switching pattern $((1,1),(2,1),(1,2),(2,2))$ results in

$$
\boldsymbol{\Theta}_{2}=\left[\begin{array}{cc}
e^{j\left(\mu_{1}+\varphi_{1}\right)} & e^{j\left(\mu_{3}+\varphi_{3}\right)} \\
e^{j\left(\mu_{2}+\varphi_{2}\right)} & e^{j\left(\mu_{4}+\varphi_{4}\right)}
\end{array}\right]
$$

The physical channel matrix $\mathbf{H}$ is, of course, unaffected by the switching pattern. The dependence of $\Theta$ on the switching pattern and on the SISO snapshot time distances is highly problematic since different switching patterns and/or SISO snapshot times yield different (incorrect) measurement results for the same physical MIMO channel. The following simple example for $M_{\mathrm{T}}=M_{\mathrm{R}}=2$ illustrates this undesirable effect and its implications: Assume that the physical channel is given by $\mathbf{H}=\mathbf{1}$, and $\mu_{1}+\varphi_{1}=\mu_{4}+\varphi_{4}=0, \mu_{2}+\varphi_{2}=-\pi / 2$, and $\mu_{3}+\varphi_{3}=\pi / 2$. It is then easily seen that $\lambda_{1}\left(\left(\mathbf{H} \circ \boldsymbol{\Theta}_{1}\right)\left(\mathbf{H} \circ \boldsymbol{\Theta}_{1}\right)^{H}\right)=\lambda_{2}\left(\left(\mathbf{H} \circ \boldsymbol{\Theta}_{1}\right)\left(\mathbf{H} \circ \boldsymbol{\Theta}_{1}\right)^{H}\right)=2$ whereas $\lambda_{1}\left(\left(\mathbf{H} \circ \boldsymbol{\Theta}_{2}\right)\left(\mathbf{H} \circ \boldsymbol{\Theta}_{2}\right)^{H}\right)=4$ 
and $\lambda_{2}\left(\left(\mathbf{H} \circ \boldsymbol{\Theta}_{2}\right)\left(\mathbf{H} \circ \boldsymbol{\Theta}_{2}\right)^{H}\right)=0$. In summary, starting from a rank-1 physical channel, depending on the switching pattern, we can get a rank-1 or a rank-2 effective channel.

We conclude this section by introducing an approximation that will frequently be used throughout the paper. For small phase errors, we use the standard first-order Taylor-series approximation (see, e.g., [29, Eq. (4.12)])

$$
\exp ^{\circ}(j \Phi) \approx \mathbf{1}+j \Phi
$$

In the remainder of the paper, whenever referring to $\widehat{\mathbf{H}}$, unless explicitly stated otherwise, we shall use the exact expression for $\Theta$ according to (20). We conclude this section by noting that, throughout the paper, whenever we deal with random physical channels, $\Theta$ will be assumed to be statistically independent of $\mathbf{H}$.

\section{EfFect of Phase ERrors on Mimo Channel Statistics}

In this section, we describe the impact of phase errors on MIMO channel statistics (i.e., the statistics of the effective MIMO channel $\widehat{\mathbf{H}}$ vs. the statistics of the physical MIMO channel $\mathbf{H}$ ) thereby laying the foundations for the results in Sections [V, VI, and VII.

In the following, we consider both deterministic and stochastic physical channels H. For deterministic $\mathbf{H}$, phase noise induces randomness and hence makes the static channel appear fading. In the case of stochastic $\mathbf{H}$, phase errors alter the channel statistics.

\section{A. Mean and Covariance of Effective MIMO Channel Matrix}

We start by investigating the impact of phase errors on the mean $\mathbf{H}_{\mathrm{f}}=\mathbb{E}\{\mathbf{H}\}$ and on the covariance $\operatorname{Cov}\{\mathbf{H}\}$ of the physical channel. The developments in the sequel apply to both deterministic physical channels, where $\mathbf{H}_{\mathrm{f}}=\mathbf{H}$ and $\operatorname{Cov}\{\mathbf{H}\}=\mathbf{0}$, and random physical 
channels. Using (20), a straightforward calculation reveals that

$$
\begin{aligned}
& \mathbb{E}\{\widehat{\mathbf{H}}\}=\sqrt{\kappa} \exp ^{\circ}(j \mathbf{M}) \circ \mathbf{H}_{\mathrm{f}} \\
& \operatorname{Cov}\{\widehat{\mathbf{H}}\}=\kappa\left(\mathbf{m m}^{H}\right) \circ {\left[\exp ^{\circ}(\operatorname{Cov}\{\boldsymbol{\Phi}\}) \circ\left(\operatorname{vec}\left(\mathbf{H}_{\mathrm{f}}\right)\left(\operatorname{vec}\left(\mathbf{H}_{\mathrm{f}}\right)\right)^{H}+\operatorname{Cov}\{\mathbf{H}\}\right)\right.} \\
&\left.-\operatorname{vec}\left(\mathbf{H}_{\mathrm{f}}\right)\left(\operatorname{vec}\left(\mathbf{H}_{\mathrm{f}}\right)\right)^{H}\right]
\end{aligned}
$$

where $\kappa=\exp \left(-\sigma_{\varphi}^{2}\right), \mathbf{m}=\operatorname{vec}\left(\exp ^{\circ}(j \mathbf{M})\right)$, and we made use of the relations $\operatorname{vec}(\mathbf{A} \circ$ B) $(\operatorname{vec}(\mathbf{A} \circ \mathbf{B}))^{H}=\left(\operatorname{vec}(\mathbf{A})(\operatorname{vec}(\mathbf{A}))^{H}\right) \circ\left(\operatorname{vec}(\mathbf{B})(\operatorname{vec}(\mathbf{B}))^{H}\right)$ and $\mathbb{E}\{\exp (j X)\}=\exp \left(j m_{X}-\right.$ $\left.\sigma_{X}^{2} / 2\right)$ for $X \sim \mathcal{N}\left(m_{X}, \sigma_{X}^{2}\right)$.

We observe that phase noise leads to an attenuation of the first moment of the physical channel by a factor of $\sqrt{\kappa}$. The presence of a frequency offset (reflected by the matrix $\mathbf{M}$ ) can result in $\mathbb{E}\{\widehat{\mathbf{H}}\}$ having a higher rank than $\mathbf{H}_{\mathrm{f}}$ which in turn implies that the spatial multiplexing gain of the effective channel can be higher than that of the underlying physical channel. Take for example a deterministic rank-1 physical channel with $\mathbf{H}=\mathbf{H}_{\mathrm{f}}=\mathbf{1}$. In the absence of phase noise, we have $\mathbb{E}\{\widehat{\mathbf{H}}\}=\exp ^{\circ}(j \mathbf{M})$ which, depending on the frequency offset characteristics, can even have full rank. As a simple example, consider the switching pattern $((1,1),(2,1),(2,2),(1,2))$ with $\mu_{k}=\exp (j \pi k / 2)$, which results in the full-rank matrix

$$
\mathbb{E}\{\widehat{\mathbf{H}}\}=\exp ^{\circ}(j \mathbf{M})=\left[\begin{array}{ll}
j & 1 \\
-1 & -j
\end{array}\right]
$$

The conditions for frequency offset (in terms of the measurement setup) to have a significant impact on the measurement error in terms of MI will be discussed in Section IV-A3,

The impact of phase errors on the channel's second-order statistics is more involved. Consider, for example, the case of no frequency offset $(\mathbf{M}=\mathbf{0}$ and hence $\mathbf{m}=\mathbf{1})$ and fully correlated 
phase noise, i.e., $\operatorname{Cov}\{\Phi\}=\sigma_{\varphi}^{2} \mathbf{1}$, representative of a high-quality LO. In this case, (22) yields

$$
\operatorname{Cov}\{\widehat{\mathbf{H}}\}=\operatorname{Cov}\{\mathbf{H}\}+\operatorname{vec}\left(\mathbf{H}_{\mathrm{f}}\right)\left(\operatorname{vec}\left(\mathbf{H}_{\mathrm{f}}\right)\right)^{H}(1-\kappa)
$$

which shows that the mere presence of phase noise, even if it is fully correlated, alters the covariance matrix of the physical channel by adding a rank-1 component to $\operatorname{Cov}\{\mathbf{H}\}$. For a deterministic physical channel, where $\operatorname{Cov}\{\mathbf{H}\}=\mathbf{0}$, we can see that the presence of phase noise randomizes the channel and yields an effective channel with the rank-1 covariance matrix $\operatorname{Cov}\{\widehat{\mathbf{H}}\}=\operatorname{vec}\left(\mathbf{H}_{\mathrm{f}}\right)\left(\operatorname{vec}\left(\mathbf{H}_{\mathrm{f}}\right)\right)^{H}(1-\kappa)$. We conclude this discussion by investigating the case of fully uncorrelated phase noise, representative of a very poor LO, still assuming $\mathbf{M}=\mathbf{0}$. In this case, we have $\boldsymbol{\Phi}=\sigma_{\varphi}^{2} \mathbf{I}$ and consequently

$$
\operatorname{Cov}\{\widehat{\mathbf{H}}\}=(1-\kappa) \operatorname{dg}\left(\operatorname{vec}\left(\mathbf{H}_{\mathrm{f}}\right)\left(\operatorname{vec}\left(\mathbf{H}_{\mathrm{f}}\right)\right)^{H}\right)+\left(\begin{array}{ccccc}
1 & \kappa & \kappa & \cdots & \kappa \\
\kappa & 1 & \kappa & \cdots & \kappa \\
\vdots & \ddots & & \ddots & \vdots \\
& & & & \\
\kappa & \kappa & \kappa & \cdots & 1
\end{array}\right) \circ \operatorname{Cov}\{\mathbf{H}\} .
$$

We can see that again for deterministic physical channels (where $\operatorname{Cov}\{\mathbf{H}\}=\mathbf{0}$ ) the effective channel has a nonzero second moment and is hence randomized by phase noise. An important effect is brought out by starting with a purely Rayleigh fading physical channel (i.e., $\mathbf{H}_{\mathrm{f}}=\mathbf{0}$ ) with fully correlated entries (due to insufficient antenna spacing for example) so that $\operatorname{Cov}\{\mathbf{H}\}=\mathbf{1}$. If the phase noise variance is high so that $\kappa=\exp \left(-\sigma_{\varphi}^{2}\right)$ is small, (23) implies that $\operatorname{Cov}\{\widehat{\mathbf{H}}\} \approx \mathbf{I}$ which amounts to a full decorrelation of the channel entries. Consequently, the effective MIMO channel "looks like" an independent and identically distributed (i.i.d.) channel. There is one subtle difference, though, to the widely used i.i.d. Rayleigh fading channel model, namely that the effective MIMO channel matrix will not have JG entries (see Section [II-B). We can therefore not conclude that the entries in the effective channel matrix are statistically independent. 
Nevertheless, the statistics of the effective channel will cause significant overestimation of the channel's MI and capacity (see, for example, the numerical results in Section IV-B). In the case of fully correlated phase noise, the effective MIMO channel matrix will have JG entries and, as discussed above, we have (assuming $\mathbf{H}_{\mathrm{f}}=\mathbf{0}$ ) $\operatorname{Cov}\{\widehat{\mathbf{H}}\}=\operatorname{Cov}\{\mathbf{H}\}$ so that overestimation does not occur.

We close this discussion by noting that we have identified a number of possible scenarios where the presence of phase errors can significantly alter the MIMO channel statistics and, in particular, can lead to substantial rank increase of the effective channel's covariance matrix with respect to (w.r.t.) the underlying physical channel's covariance matrix. From a channel measurement point of view, the consequences are significant measurement errors in terms of MI and capacity. Corresponding quantitative results (analytic and numerical) will be provided in Sections $[\mathrm{IV}$ and VI. Finally, we recall that the situation is exacerbated by the fact that measurement errors depend significantly on the antenna switching pattern as well as the SISO snapshot time distances.

\section{B. Loss of (Joint and Individual) Gaussianity}

The impact of phase noise on the MIMO channel statistics is not restricted to the first and second moments as discussed above. Rather, as shown below, it affects the joint and individual distributions of the scalar subchannels in a profound way. In the following, for the sake of clarity of exposition, we set $\mathbf{M}=\mathbf{0}$.

1) Effect on the joint distribution of the Rayleigh fading physical channel elements: We start by assuming that the physical channel is Rayleigh distributed, i.e., $\mathbf{H}_{\mathrm{f}}=\mathbb{E}\{\mathbf{H}\}=\mathbf{0}$ and $\mathbf{H}$ is a zero-mean proper (or equivalently "circularly symmetric”) complex Gaussian random matrix. We

shall next show that even though the individual entries of $\widehat{\mathbf{H}}$ continue to be circularly-symmetric complex Gaussian, they will, in general, not be JG. The first part of this statement is made precise in the following Lemma for which, even though it is straightforward, we could not find a reference in the literature. 
Lemma 1: Let $h \stackrel{\mathrm{d}}{=} \mathcal{C N}\left(0, \sigma_{h}^{2}\right)$ and take $\varphi$ to be a continuous $\mathrm{RV}$ with pdf $\mathrm{p}_{\varphi}(x)$. The $\mathrm{RV}$ $h \exp (j \varphi)$ is $\mathcal{C N}\left(0, \sigma_{h}^{2}\right)$, irrespective of $\mathrm{p}_{\varphi}(x)$.

Proof: Since $|\exp (j \varphi)|=1$, it follows immediately that the pdf of $|h \exp (j \varphi)|$ is equal to the pdf of $|h|$. The statistics of the phase of $h \exp (j \varphi)$ are obtained by noting that $\arg (h \exp (j \varphi))=$ $(\arg (h)+\varphi) \bmod 2 \pi=\tilde{z}$ with $\arg (h)$ being uniformly distributed in $[0,2 \pi)$. Our task is therefore reduced to finding the pdf $\mathrm{p}_{\tilde{z}}(x)$. Next, denote the $2 \pi$-periodic continuation of the pdf of $\arg (h)$ and of $\varphi$ as $\mathrm{p}_{\tilde{h}}(x)=1 /(2 \pi)$ and $\mathrm{p}_{\tilde{\varphi}}(x)=\sum_{l=-\infty}^{\infty} \mathrm{p}_{\varphi}(x-2 \pi l)$, respectively. Noting that $\arg (h)$ and $\varphi$ are statistically independent, we obtain $\mathrm{p}_{\tilde{z}}(x)=\int_{-\pi}^{\pi} \mathrm{p}_{\tilde{h}}(x) \mathrm{p}_{\tilde{\varphi}}(y-x) \mathrm{d} x=$ $1 /(2 \pi) \int_{-\pi}^{\pi} \mathrm{p}_{\tilde{\varphi}}(y-x) \mathrm{d} x=1 /(2 \pi)$.

It remains to show that the elements in $\widehat{\mathbf{H}}$ will, in general, not be JG. This will be done by considering a simple example. Since two complex Gaussian RVs $x_{1}$ and $x_{2}$ are JG if and only if the linear combination $a x_{1}+b x_{2}$ is (complex) Gaussian $\forall\{a, b\} \in \mathbb{C}$, it suffices to show that $z=h_{1} \exp \left(j \varphi_{1}\right)+h_{2} \exp \left(j \varphi_{2}\right)$ with $h_{1}, h_{2} \stackrel{\mathrm{d}}{=} \mathcal{C N}(0,1)$ will not be Gaussian if $h_{1}$ and $h_{2}$ are fully correlated, i.e., $h_{1}=h_{2}$ and hence $z=h_{1} y$ where $y=\exp \left(j \varphi_{1}\right)+\exp \left(j \varphi_{2}\right)$. A simple proof is obtained by writing the MGF of $z$ as $M_{z}(s)=\mathbb{E}\{\exp (s z)\}=\mathbb{E}_{y} \mathbb{E}_{h_{1} \mid y}\left\{\exp \left(s h_{1} y\right)\right\}=$ $\mathbb{E}_{y}\left\{\exp \left(|y|^{2}|s|^{2} / 4\right)\right\}$ which is the MGF of a complex Gaussian RV only if $|y|$ is deterministic (which is satisfied for $\varphi_{1}$ and $\varphi_{2}$ fully correlated or both being deterministic, but not in general).

2) Effect on the joint distribution of the Ricean fading physical channel elements: Next, we consider a Ricean fading physical channel according to $\mathbf{H} \stackrel{\mathrm{d}}{=} \mathcal{C N}\left(\mathbf{H}_{\mathrm{f}}, \boldsymbol{\Sigma}_{\mathbf{H}}\right)$, where $\mathbf{H}_{\mathrm{f}} \neq 0$. We shall show that the presence of phase noise results not only in a loss of joint Gaussianity of the elements of $\widehat{\mathbf{H}}$, as in the Rayleigh fading case, but also in a loss of properness and Gaussianity of the individual entries in $\widehat{\mathbf{H}}$. The loss of properness follows by noting that

$$
\begin{aligned}
\operatorname{Cov}_{p}\{\hat{h}\} & =\mathbb{E}\left\{(\hat{h}-\mathbb{E}\{\hat{h}\})^{2}\right\}=\mathbb{E}\left\{\hat{h}^{2}\right\}-(\mathbb{E}\{\hat{h}\})^{2}=\mathbb{E}\left\{h^{2} e^{j 2 \varphi}\right\}-\left(\sqrt{\kappa} h_{f}\right)^{2} \\
& =h_{f}^{2} \kappa^{2}-\kappa h_{f}^{2}=h_{f}^{2}\left(\kappa^{2}-\kappa\right) \neq 0
\end{aligned}
$$


for $\sigma_{\varphi}^{2}>0$ and hence $\kappa<1$. It follows immediately that the elements of $\widehat{\mathbf{H}}$ are no more jointly proper as well. The loss of Gaussianity is a direct consequence of $h_{\mathrm{f}} e^{j \varphi}$ not being Gaussian distributed. Since the marginals are not Gaussian, we can immediately conclude that the elements of $\mathbf{H}$ will not be $\mathrm{JG}$ either.

3) Special cases: We conclude this discussion by dropping the assumption $\mathbf{M}=\mathbf{0}$ and identifying two interesting special cases where, despite the presence of phase errors, the effective MIMO channel matrix has JG entries.

The first case is that of a deterministic physical channel $\mathbf{H}=\mathbf{H}_{\mathrm{f}}$ subject to "small" (in the sense of approximation (21) being appropriate) phase noise. More specifically, we have

$$
\widehat{\mathbf{H}}=\mathbf{H}_{\mathrm{f}} \circ \exp ^{\circ}(j \mathbf{M}) \circ(\mathbf{1}+j \boldsymbol{\Phi}) .
$$

Since the entries in $\Phi$ are samples of a zero-mean (real) Gaussian process, any finite set of such samples is zero-mean JG which results in the entries of $\widehat{\mathbf{H}}$ being JG. The corresponding first and second moments are given by

$$
\begin{aligned}
\mathbb{E}\{\widehat{\mathbf{H}}\} & =\mathbf{H}_{\mathrm{f}} \circ \exp ^{\circ}(j \mathbf{M}) \\
\operatorname{Cov}\{\widehat{\mathbf{H}}\} & =\left(\mathbf{m m}^{H}\right) \circ\left(\operatorname{vec}\left(\mathbf{H}_{\mathrm{f}}\right)\left(\operatorname{vec}\left(\mathbf{H}_{\mathrm{f}}\right)\right)^{H}\right) \circ \operatorname{Cov}\{\boldsymbol{\Phi}\} .
\end{aligned}
$$

Note that while the entries in $\widehat{\mathbf{H}}$ are $\mathrm{JG}$, they will neither be jointly proper nor individually proper in general so that the second-order description in (24) is incomplete without specifying the pseudo-covariance matrix obtained as

$$
\operatorname{Cov}_{\mathrm{p}}\{\widehat{\mathbf{H}}\}=-\left(\mathbf{m m}^{T}\right) \circ\left(\operatorname{vec}\left(\mathbf{H}_{\mathrm{f}}\right)\left(\operatorname{vec}\left(\mathbf{H}_{\mathrm{f}}\right)\right)^{T}\right) \circ \operatorname{Cov}\{\boldsymbol{\Phi}\} .
$$

The second special case is that of an i.i.d. purely Rayleigh fading physical channel $\mathbf{H}$. Defining $\mathbf{h}=\operatorname{vec}(\mathbf{H}) \stackrel{\mathrm{d}}{=} \mathcal{C N}(\mathbf{0}, \mathbf{I})$, we want to show that $\mathbf{D h} \stackrel{\mathrm{d}}{=} \mathcal{C N}(\mathbf{0}, \mathbf{I})$, where $\mathbf{D}=$ $\operatorname{diag}\left(\left[\exp \left(j\left(\mu_{1}+\varphi_{1}\right)\right) \exp \left(j\left(\mu_{2}+\varphi_{2}\right)\right) \quad \cdots \quad \exp \left(j\left(\mu_{M_{\mathrm{T}} M_{\mathrm{R}}}+\varphi_{M_{\mathrm{T}} M_{\mathrm{R}}}\right)\right)\right]\right)$. We start by noting 
that the characteristic function of $\mathbf{h}$ is given by [30, Eq. (20)]

$$
\Psi_{\mathbf{h}}(j \boldsymbol{\nu})=\mathbb{E}\left\{e^{j \operatorname{Re}\left(\mathbf{h}^{H} \boldsymbol{\nu}\right)}\right\}=e^{-\boldsymbol{\nu}^{H} \boldsymbol{\nu} / 4}, \quad \boldsymbol{\nu} \in \mathbb{C}^{M_{\mathrm{T}} M_{\mathrm{R}}}
$$

The characteristic function of $\mathrm{Dh}$ is obtained as

$$
\begin{aligned}
\Psi_{\mathbf{D h}}(j \boldsymbol{\nu}) & =\mathbb{E}\left\{e^{j \operatorname{Re}\left(\mathbf{h}^{H} \mathbf{D}^{H} \boldsymbol{\nu}\right)}\right\}=\mathbb{E}_{\mathbf{D}}\left\{\mathbb{E}_{\mathbf{h} \mid \mathbf{D}}\left\{e^{j \operatorname{Re}\left(\mathbf{h}^{H} \mathbf{D}^{H} \boldsymbol{\nu}\right)}\right\}\right\} \\
& =\mathbb{E}_{\mathbf{D}}\left\{e^{-\boldsymbol{\nu}^{H} \mathbf{D} \mathbf{D}^{H} \boldsymbol{\nu} / 4}\right\}=\mathbb{E}_{\mathbf{D}}\left\{e^{-\boldsymbol{\nu}^{H} \boldsymbol{\nu} / 4}\right\}=e^{-\boldsymbol{\nu}^{H} \boldsymbol{\nu} / 4}=\Psi_{\mathbf{h}}(j \boldsymbol{\nu})
\end{aligned}
$$

where we made use of the fact that $\mathbf{D D}^{H}=\mathbf{I}$. We have therefore shown that i.i.d. purely Rayleigh fading physical channels are not affected by phase errors.

In closing, we would like to remark that the effective channel not having JG entries, in general, is one of the main factors contributing to the difficulties in analyzing the impact of phase errors on MI.

\section{EfFect of Phase Errors on Mutual Information}

Having seen in the previous section that phase errors can alter the MIMO channel statistics significantly, the purpose of this section is to analyze the corresponding impact on MI for random physical channels. Analytic results for the general (arbitrary rank (with probability (w.p.) 1) of the physical channel) case seem very difficult to obtain. We shall therefore restrict our discussion to identifying cases where the MI is not affected even though the channel statistics are. In addition, representative numerical results bringing out the key consequences of phase errors will be provided. Analytic results for deterministic physical channels and for (deterministic or random) rank-1 physical channels will be provided in Sections $\mathrm{V}$ and $\mathrm{VI}$, respectively.

We analyze a MIMO channel with input-output relation

$$
\mathbf{r}=\mathbf{H s}+\mathbf{n}
$$

where $\mathbf{s}$ is the $M_{\mathrm{T}} \times 1$ transmit vector, $\mathbf{r}$ is the $M_{\mathrm{R}} \times 1$ receive vector, and $\mathbf{n}$ is an $M_{\mathrm{R}} \times 1$ 
noise vector distributed as $\mathcal{C N}\left(\mathbf{0}, \mathbf{I}_{M_{\mathrm{R}}}\right)$. Assuming no channel state information (CSI) at the transmitter and perfect CSI at the receiver, the MI (in bit/s/Hz) of this channel is given by [31]

$$
I=\log \operatorname{det}\left(\mathbf{I}_{M_{\mathrm{R}}}+\frac{\rho}{M_{\mathrm{T}}} \mathbf{H} \mathbf{H}^{H}\right)=\log \operatorname{det}\left(\mathbf{I}_{M_{\mathrm{T}}}+\frac{\rho}{M_{\mathrm{T}}} \mathbf{H}^{H} \mathbf{H}\right)
$$

where the input signal vector was assumed to be circularly-symmetric complex Gaussian with covariance matrix $\left(\rho / M_{\mathrm{T}}\right) \mathbf{I}_{M_{\mathrm{T}}}$ and $\rho$ is the average SNR at each of the receive antennas.

The purpose of this section is to study how $I$ changes when $\mathbf{H}$ in $(25)$ is replaced by $\widehat{\mathbf{H}}$ in $(20)$, i.e., to analyze the statistics of $\hat{I}=\log \operatorname{det}\left(\mathbf{I}_{M_{\mathrm{R}}}+\left(\rho / M_{\mathrm{T}}\right) \widehat{\mathbf{H}} \widehat{\mathbf{H}}^{H}\right)=\log \operatorname{det}\left(\mathbf{I}_{M_{\mathrm{T}}}+\left(\rho / M_{\mathrm{T}}\right) \widehat{\mathbf{H}}^{H} \widehat{\mathbf{H}}\right)$.

\section{A. Cases Where Mutual Information is Not Affected}

In the last paragraph of Section III, we showed that i.i.d. Rayleigh fading channels are not affected by phase errors in the sense that the effective channel $\widehat{\mathbf{H}}$ is i.i.d. Rayleigh fading as well, irrespectively of the statistics of the phase errors. We have furthermore seen (Section $\amalg I I-A)$ that for correlated Rayleigh or Ricean fading physical channels, phase errors can have a significant impact on the channel statistics and thus on the corresponding MI. There are cases, however, where even though the statistics of the effective channel $\widehat{\mathbf{H}}$ differ from the statistics of $\mathbf{H}$, we still have $\hat{I}=I$. Intuitively, this happens because of the quadratic dependence of $\hat{I}$ on $\widehat{\mathbf{H}}$. In the following, we shall discuss two such practically very relevant cases.

1) The low-SNR regime: Phase errors have no impact on MI in the low-SNR regime, irrespectively of the physical channel's statistics and the phase error statistics. This can easily be seen by noting that for low SNR

$$
I \approx \log \left(1+\frac{\rho}{M_{\mathrm{T}}}\|\mathbf{H}\|_{\mathrm{F}}^{2}\right)
$$

which, combined with the fact that $\|\mathbf{H}\|_{\mathrm{F}}^{2}=\|\widehat{\mathbf{H}}\|_{\mathrm{F}}^{2}$, proves the statement. In general, we can conclude that the impact of phase errors on MI is more pronounced for higher SNR. This is because phase errors lead to a rank increase of the MIMO channel and high-SNR MI depends 
strongly on the rank (or multiplexing gain) of the channel, whereas low-SNR MI depends only on the Frobenius norm $\|\mathbf{H}\|_{\mathrm{F}}$.

2) One-sided switching or fully parallel sounding: For MIMO channel sounders where either the transmitter or the receiver employs one RF chain per antenna (i.e., parallel sounding) and hence no switching is necessary on the corresponding side of the link, the effective channel matrix is given by $\widehat{\mathbf{H}}=\mathrm{D}_{\mathrm{R}} \mathbf{H} d_{\mathrm{T}}$ and $\widehat{\mathbf{H}}=d_{\mathrm{R}} \mathbf{H D}_{\mathrm{T}}$ in the case of switching only at the receive and the transmit side, respectively. Here, $d_{\mathrm{R}}$ and $d_{\mathrm{T}}$ as well as the entries of the diagonal matrices $\mathbf{D}_{\mathrm{R}}$ and $\mathbf{D}_{\mathrm{T}}$ are of the form $\exp (j(\mu+\varphi))$. Even though (22) implies that the statistics of the effective channel $\widehat{\mathbf{H}}$ are different from the statistics of the physical channel $\mathbf{H}$, it is easily seen by direct insertion into (25) that the MI is not affected by "one-sided" phase errors. Obviously, this is also true for fully parallel (both the transmitter and the receiver employ one RF chain per antenna) MIMO channel sounders.

We would like to add a word of caution. In practice, the MI of measured MIMO channels is often not evaluated by directly inserting the measured channel realizations into the MI formula (25). Rather, the measurement results are used to extract parameters of a statistical MIMO channel model, e.g., the power-angular spectrum (PAS) or the distribution of the DOA. The resulting statistical description of $\mathbf{H}$ is then used to evaluate the channel's MI. Now, one-sided switching does, in general, entail errors in the estimation of the parameters of a statistical MIMO channel model so that the procedure described above will lead to, in general significant, errors in MI. This brings out an interesting and practically very relevant point. While one-sided switching does not entail errors in MI when the measurement results are used to directly evaluate the MI, significant errors can be expected if one takes a detour via a specific statistical MIMO channel model.

3) Impact of frequency offset for separable timing matrix: First, we define the timing matrix $[\mathbf{T}]_{m_{k}, n_{k}}=t_{k}$ of size $M_{\mathrm{R}} \times M_{\mathrm{T}}$, which contains the SISO snapshot measurement times in matrix form arranged corresponding to the switching pattern. We call $\mathbf{T}$ separable if it can be written 
in the form $[\mathbf{T}]_{m, n}=\left[\mathbf{t}_{\mathrm{R}}\right]_{m}+\left[\mathbf{t}_{\mathrm{T}}\right]_{n}$, where $\mathbf{t}_{\mathrm{T}}$ and $\mathbf{t}_{\mathrm{R}}$ are the transmit and receive timing vectors of size $M_{\mathrm{T}} \times 1$ and $M_{\mathrm{R}} \times 1$, respectively. An example of a switching pattern and SISO snapshot times leading to a separable timing matrix is given by the regular sounding pattern (where sounding pattern denotes the combination of a switching pattern and a set of SISO snapshot times)

$$
\begin{aligned}
m_{k} & =(k-1) \bmod M_{\mathrm{R}}+1 \\
n_{k} & =(k-1) \operatorname{div} M_{\mathrm{R}}+1 \\
t_{k} & =T_{\mathrm{R}}\left(m_{k}-1\right)+T_{\mathrm{T}}\left(n_{k}-1\right)
\end{aligned}
$$

with $k=1,2, \ldots, M_{\mathrm{T}} M_{\mathrm{R}}$, characterized by the timing parameters $T_{\mathrm{T}}, T_{\mathrm{R}} \in \mathbb{R}^{+}$. This corresponds to starting with transmit antenna 1 , switching through the receive antennas $1,2, \ldots, M_{\mathrm{R}}$ sequentially, then switching to transmit antenna 2, again switching through the receive antennas sequentially with the same SISO snapshot time distances between the receive antennas as before, and so on. Likewise, if we start with receive antenna 1, switch through the transmit antennas sequentially and so on, a separable timing matrix will be obtained.

Now, for $[\mathbf{T}]_{m, n}=\left[\mathbf{t}_{\mathrm{R}}\right]_{m}+\left[\mathbf{t}_{\mathrm{T}}\right]_{n}$, we have $\exp ^{\circ}(j \mathbf{M})=\exp ^{\circ}\left(j \Delta \omega \mathbf{t}_{\mathrm{R}}\right)\left(\exp ^{\circ}\left(j \Delta \omega \mathbf{t}_{\mathrm{T}}\right)\right)^{T}$ and hence by Theorem 4 in Section $\mathbb{\text { VI }}$, it follows that the eigenvalues of $\mathbf{H} \circ \exp ^{\circ}(j(\mathbf{M}+\boldsymbol{\Phi}))$ are equal to the eigenvalues of $\mathbf{H} \circ \exp ^{\circ}(j \boldsymbol{\Phi})$. This implies that for $\mathbf{T}$ separable, the frequency offset has no effect on MI. In practice, one typically has control over the sounding pattern. In the light of what was said above, it is therefore sensible to choose the sounding pattern such that $\mathbf{T}$ is separable. Of course, even if MI is not affected, frequency offset can still cause significant errors in other parameters, as discussed in the last paragraph of Section IV-A2. We note, however, that in contrast to phase noise, frequency offset, due to its deterministic nature, can be estimated and mitigated with relative ease [5]. In the remainder of the paper, we shall, therefore, often neglect the frequency offset and consider $\mathbf{H} \circ \exp ^{\circ}(j \boldsymbol{\Phi})$ only. 


\section{B. Numerical Results}

We shall next provide numerical results to quantify the impact of phase errors on MI. In particular, we will also quantify the impact of the scalar subchannels in the effective MIMO channel not being JG distributed.

1) Ergodic capacity increase due to phase errors: We examine an $M_{\mathrm{T}}=M_{\mathrm{R}}=M=8$ physical channel (with receive antenna correlation) given by $\mathbf{H}=\mathbf{R}^{1 / 2} \mathbf{H}_{\mathrm{w}}$, where $\mathbf{R}=\mathbf{R}^{1 / 2} \mathbf{R}^{1 / 2}$ denotes the receive correlation matrix and the entries of $\mathbf{H}_{\mathrm{w}}$ are i.i.d. $\mathcal{C N}(0,1)$. We choose $\mathbf{R}$ such that $\lambda_{i}(\mathbf{R})=M / \mathrm{r}(\mathbf{R})$ for $i=1,2, \ldots, \mathrm{r}(\mathbf{R})$ and $\lambda_{i}(\mathbf{R})=0$ otherwise. This normalization ensures that $\operatorname{Tr}(\mathbf{R})=M$, irrespectively of the rank of $\mathbf{R}$. Employing Monte Carlo simulations, Fig. 2 shows the ergodic capacity $6 \widehat{C}=\mathbb{E}\{\hat{I}\}$ of the effective channel $\widehat{\mathbf{H}}$ as a function of SNR for varying $r(\mathbf{R})$, varying $\sigma_{\varphi}^{2}$ with fully uncorrelated (i.e., worst-case) phase noise, i.e., $\operatorname{Cov}\{\boldsymbol{\Phi}\}=$ $\sigma_{\varphi}^{2} \mathbf{I}_{M_{\mathrm{T}} M_{\mathrm{R}}}$, and no frequency offset. We have chosen $3.5^{\circ}$ and $7^{\circ} \mathrm{rms}$ phase noise (corresponding to $\sigma_{\varphi}^{2} \approx 0.0037$ and $\sigma_{\varphi}^{2} \approx 0.0149$, respectively) as typical and worst case values, respectively. These values were derived from our measurements on a commercially employed TDMS-based MIMO channel sounder (see Section VII). In general, phase noise correlation properties quantified by $\operatorname{Cov}\{\boldsymbol{\Phi}\}$ and phase noise variance $\sigma_{\varphi}^{2}$ depend on the LO characteristics, SISO snapshot times, and the number of transmit and receive antennas. Fully uncorrelated phase noise, as assumed in this example, corresponds to relatively long SISO snapshot time distances and represents the worst (though not necessarily untypical) conditions. We can see in Fig. 2, that in none of the considered cases phase noise results in a reduction of ergodic capacity. Moreover, we observe, in agreement with what was shown in Section $\amalg$ III-B3, that in the case of i.i.d. physical channels (i.e., $r(\mathbf{R})=8$ in this example 7 ) phase noise has no impact at all on ergodic capacity. In the $\mathrm{r}(\mathbf{R})=1$ case, at $\rho=35 \mathrm{~dB}$ for the typical rms phase noise value of $3.5^{\circ}$, the error in ergodic

\footnotetext{
${ }^{6}$ Throughout the paper, we tacitly assume that the effective channel is ergodic.

${ }^{7}$ Recall that the nonzero eigenvalues of $\mathbf{R}$ were chosen to be equal, which together with $r(\mathbf{R})=8$ implies that the entries of $\mathbf{H}=\mathbf{R}^{1 / 2} \mathbf{H}_{\mathrm{w}}$ are i.i.d. $\mathcal{C} \mathcal{N}(0,1)$.
} 
capacity due to phase noise is about $100 \%$. For the worst-case value of $7^{\circ} \mathrm{rms}$ phase noise, the error, again at $\rho=35 \mathrm{~dB}$, is about $175 \%$. Furthermore, for $\mathrm{r}(\mathbf{R})=1$ and rms phase noise of $3.5^{\circ}$, the multiplexing gain of the effective channel (i.e., the ergodic capacity pre-log, obtained by determining the high-SNR slope of capacity as a function of SNR) lies between 4 and 8, making the effective channel look like a MIMO channel with rank between 4 and 8 . Furthermore, as predicted by theory, we can see that phase noise has little impact in the low-SNR regime. We conclude this simulation example by noting that the impact of phase noise is most pronounced for low-rank physical channels at high SNR. This is one of the motivating factors for the detailed analysis of the rank-1 physical channel in Section VI.

2) Impact of loss of joint Gaussianity: As demonstrated in Section [I-B albeit the individual elements of the effective MIMO channel associated with a physical purely Rayleigh fading channel will be circularly-symmetric complex Gaussian distributed, they will, in general, not be JG distributed. Characterizing the consequences of this loss of joint Gaussianity analytically seems very difficult as known techniques (e.g., [32]-[37]) for deriving analytic expressions for ergodic and outage capacity of MIMO channels (or bounds thereon) almost exclusively hinge on the assumption of the elements in $\mathbf{H}$ being $\mathrm{JG}$ distributed. There are two practically relevant points related to the loss of joint Gaussianity, which will be brought out through numerical results in the following.

We investigate a $4 \times 4$ purely Rayleigh fading (i.e., $\mathbf{H}_{\mathrm{f}}=\mathbf{0}$ ) physical channel with two different correlation levels according to $[\operatorname{Corr}\{\mathbf{H}\}]_{m, n}=0.7$ and 0.95 , respectively, for $m \neq n$ in the presence of fully uncorrelated $7^{\circ} \mathrm{rms}$ phase noise and no frequency offset. For $\rho=20 \mathrm{~dB}$, Fig. 3 shows the cdf of MI for the physical channel $\mathbf{H}$, the resulting effective channel $\widehat{\mathbf{H}}$, and a synthetic MIMO channel obtained by assuming that the channel matrix is circularly-symmetric complex Gaussian with covariance matrix according to $(\underline{23})$, i.e., $[\operatorname{Cov}\{\widehat{\mathbf{H}}\}]_{m, n}=0.7 \kappa$ and $0.95 \kappa$, respectively, for $m \neq n$. In addition, the cdf of MI under the linear phase noise approximation (21) is shown. Before proceeding, we note that the synthetic channel has the same first and 
second order statistics as the effective channel and should hence exhibit (when compared to the effective channel) the impact of the loss of joint Gaussianity due to phase errors.

We can now draw the following conclusions for this specific setup:

- The impact of phase noise is significant at the (high) correlation level of 0.95. In addition, comparing the synthetic channel to the effective channel, we can see that neglecting the loss of joint Gaussianity would result in a slight overestimation and a significant underestimation of outage capacity at low and high outage levels, respectively. For outage levels of practical interest, i.e., up to $30 \%$ outage probability, the error in outage capacity (due to the loss of joint Gaussianity) is not more than $8 \%$.

- The impact of phase noise is negligible at the correlation level of 0.7. The synthetic channel exhibits a behavior that is very close to that of the effective channel. Interestingly, a correlation level of 0.7-0.75 is often quoted in the literature [38], [39] as a threshold above which "the channel starts behaving as highly correlated".

- Even for the worst case rms phase noise value of $7^{\circ}$, the linear phase noise approximation (21) yields very accurate results. This approximation will be used extensively in Section VI.

With regards to Ricean fading physical channels, we content ourselves with noting that for high Ricean K-factors, the situation approaches that for deterministic physical channels (studied in Sections $\nabla$ and $(\nabla I)$, whereas for low Ricean K-factors, the behavior will be close to that of the purely Rayleigh fading case (treated in this section and in Section VI).

\section{Sensitivity Analysis}

Throughout this section, we assume a separable timing matrix in which case frequency offset has no effect on MI and can therefore be neglected. For the sake of simplicity of exposition, we shall furthermore assume $M_{\mathrm{R}} \leq M_{\mathrm{T}}$. The purpose of this section is twofold. First, we introduce a tool for evaluating the sensitivity of the MI of a fixed physical channel to phase noise. This will be accomplished by computing the first two terms in the Taylor series expansion of $\hat{I}(\boldsymbol{\Phi})$ 
around the phase-noise-free case $\Phi=0$. Based on this framework, we will then be able to provide analytic expressions for approximations of the first and second moment of the MI of the effective channel for arbitrary phase noise covariance matrix.

We shall be concerned with computing the second-order Taylor series expansion of

$$
\hat{I}(\boldsymbol{\Phi})=\log \operatorname{det}\left(\mathbf{I}_{M_{\mathrm{R}}}+\frac{\rho}{M_{\mathrm{T}}}\left(\mathbf{H} \circ \exp ^{\circ}(j \boldsymbol{\Phi})\right)\left(\mathbf{H} \circ \exp ^{\circ}(j \boldsymbol{\Phi})\right)^{H}\right)
$$

around $\Phi=\mathbf{0}$ given by

$$
\tilde{I}(\boldsymbol{\Phi})=\hat{I}(\mathbf{0})+\mathcal{J}_{\hat{I}}(\mathbf{0}) \operatorname{vec}(\boldsymbol{\Phi})+\frac{1}{2}(\operatorname{vec}(\boldsymbol{\Phi}))^{T} \mathcal{H}_{\hat{I}}(\mathbf{0}) \operatorname{vec}(\boldsymbol{\Phi})
$$

where $\mathcal{J}_{\hat{I}}(\mathbf{0})$ denotes the $1 \times M_{\mathrm{T}} M_{\mathrm{R}}$ Jacobian matrix (vector, in this case) and $\mathcal{H}_{\hat{I}}(\mathbf{0})$ is the $M_{\mathrm{T}} M_{\mathrm{R}} \times M_{\mathrm{T}} M_{\mathrm{R}}$ Hessian matrix of $\hat{I}=\hat{I}(\boldsymbol{\Phi})$ at $\boldsymbol{\Phi}=\mathbf{0}$. Clearly, we have $\hat{I}(\mathbf{0})=I$. We shall see below that the second-order Taylor series expansion of MI is, in general, accurate for full-rank physical channels, but tends to yield loose approximations for rank-deficient physical channels. This problem can be mitigated by either using more terms in the Taylor series expansion of MI or by performing the Taylor series expansion on the channel's eigenvalues rather than on the channel's MI itself. Both approaches are, in general, cumbersome. The latter approach will not be detailed here, for brevity of exposition, but will be outlined briefly in Section $\mathrm{V}-\mathrm{C}$. Even though the second-order Taylor series expansion of MI does not yield accurate approximations in the case of rank-deficient physical channels, explicit expressions for the Jacobian matrix $\mathcal{J}_{\hat{I}}(\mathbf{0})$ and the Hessian matrix $\mathcal{H}_{\hat{I}}(\mathbf{0})$ can be used to test whether $\hat{I}(\boldsymbol{\Phi})$ has an extremum at $\mathbf{\Phi}=\mathbf{0}$.

\section{A. Sensitivity Analysis}

Even though the computation of the Jacobian matrix $\mathcal{J}_{\hat{I}}(\mathbf{0})$ and the Hessian matrix $\mathcal{H}_{\hat{I}}(\mathbf{0})$ does not pose any major technical difficulties, it still requires the application of tools that are not completely standard, namely matrix differential calculus [12] and matrix-variate Wirtinger a.k.a. $\mathbb{C R}$ calculus as described in [40]. We shall therefore present the corresponding derivations 
in some detail.

Theorem 1: The $1 \times M_{\mathrm{T}} M_{\mathrm{R}}$ Jacobian matrix (vector) $\mathcal{J}_{\hat{I}}(\mathbf{0})$ in (27) is given by

$$
\mathcal{J}_{\hat{I}}(\mathbf{0})=2 \log (e) \operatorname{Im}\left(\left(\operatorname{vec}\left(\left(\mathbf{Y}^{-1} \mathbf{H}\right) \circ \mathbf{H}^{*}\right)\right)^{T}\right)
$$

where

$$
\mathbf{Y}=\frac{M_{\mathrm{T}}}{\rho} \mathbf{I}_{M_{\mathrm{R}}}+\mathbf{H H}^{H}
$$

The $M_{\mathrm{T}} M_{\mathrm{R}} \times M_{\mathrm{T}} M_{\mathrm{R}}$ Hessian matrix $\mathcal{H}_{\hat{I}}(\mathbf{0})$ in (27) is given by

$$
\begin{aligned}
\mathcal{H}_{\hat{I}}(\mathbf{0})=2 \log (e) \operatorname{Re}( & \operatorname{divec}(\mathbf{H}) \mathbf{K}_{\left(M_{\mathrm{T}}, M_{\mathrm{R}}\right)}\left(\left(\mathbf{H}^{H} \mathbf{Y}^{-1}\right)^{T} \otimes \mathbf{H}^{H} \mathbf{Y}^{-1}\right) \operatorname{divec}(\mathbf{H}) \\
& +\operatorname{divec}(\mathbf{H})\left(\left(\mathbf{I}_{M_{\mathrm{T}}}-\mathbf{H}^{H} \mathbf{Y}^{-1} \mathbf{H}\right) \otimes\left(\mathbf{Y}^{-1}\right)^{T}\right) \operatorname{divec}\left(\mathbf{H}^{*}\right) \\
& \left.-\operatorname{divec}\left(\left(\mathbf{Y}^{-1} \mathbf{H}\right) \circ \mathbf{H}^{*}\right)\right)
\end{aligned}
$$

Proof: We start by defining

$$
\widehat{\mathbf{Y}}=\frac{M_{\mathrm{T}}}{\rho} \mathbf{I}_{M_{\mathrm{R}}}+\underbrace{\left(\mathbf{H} \circ \exp ^{\circ}(j \boldsymbol{\Phi})\right)}_{\widehat{\mathbf{H}}} \underbrace{\left(\mathbf{H} \circ \exp ^{\circ}(j \boldsymbol{\Phi})\right)^{H}}_{\widehat{\mathbf{H}}^{H}}
$$

so that $\mathbf{Y}=\left.\widehat{\mathbf{Y}}\right|_{\mathbf{\Phi}=\mathbf{0}}$ and hence $\hat{I}=\log \operatorname{det}\left(\left(\rho / M_{\mathrm{T}}\right) \widehat{\mathbf{Y}}\right)$. The strategy used in the proof of both parts of the statement is to compute $\mathrm{d} \hat{I}$ and $\mathrm{d}^{2} \hat{I}$ and to bring the resulting expressions into the form

$$
\begin{aligned}
\mathrm{d} \hat{I} & =\mathbf{A} \operatorname{vec}(\mathrm{d} \boldsymbol{\Phi}) \\
\mathrm{d}^{2} \hat{I} & =(\operatorname{vec}(\mathrm{d} \boldsymbol{\Phi}))^{T} \mathbf{B} \operatorname{vec}(\mathrm{d} \boldsymbol{\Phi})
\end{aligned}
$$

which will then allow us to apply the first $[12$, Ch. 5, Th. 6] and the second [12, Ch. 6, Th. 6] 
identification theorem for a real-valued function of real-valued parameters to conclude that

$$
\mathcal{J}_{\hat{I}}(\mathbf{0})=\mathbf{A} \quad \text { and } \quad \mathcal{H}_{\hat{I}}(\mathbf{0})=\frac{1}{2}\left(\mathbf{B}+\mathbf{B}^{T}\right)
$$

Computing the Jacobian matrix: Using the basic rules of differentiation together with the relation $\mathrm{d} \log _{e} \operatorname{det}(\mathbf{A})=\operatorname{Tr}\left(\mathbf{A}^{-1} \mathrm{~d} \mathbf{A}\right)[12$, Sec. 8.3, Eq. (11)], we obtain

$$
\mathrm{d} \log \operatorname{det}\left(\left(\rho / M_{\mathrm{T}}\right) \widehat{\mathbf{Y}}\right)=\log (e) \operatorname{Tr}\left(\widehat{\mathbf{Y}}^{-1} \mathrm{~d} \widehat{\mathbf{Y}}\right)
$$

Applying the product rule $\mathrm{d}(\mathbf{A B})=(\mathrm{d} \mathbf{A}) \mathbf{B}+\mathbf{A d} \mathbf{B}\left[12\right.$, Sec. 8.2, Eq. (15)] and $\mathrm{d}\left(\mathbf{A}^{T}\right)=(\mathrm{d} \mathbf{A})^{T}$ [12, Sec. 8.2, Eq. (18)], we get, using Wirtinger calculus,

$$
\mathrm{d} \widehat{\mathbf{Y}}=\mathrm{d}\left(\widehat{\mathbf{H}} \widehat{\mathbf{H}}^{H}\right)=(\mathrm{d} \widehat{\mathbf{H}}) \widehat{\mathbf{H}}^{H}+\widehat{\mathbf{H}}\left(\mathrm{d}\left(\widehat{\mathbf{H}}^{*}\right)\right)^{T}
$$

With $\mathrm{d}(\mathbf{A} \circ \mathbf{B})=(\mathrm{d} \mathbf{A}) \circ \mathbf{B}+\mathbf{A} \circ(\mathrm{d} \mathbf{B})[12$, Sec. 8.2, Eq. (17)], we have $\mathrm{d} \widehat{\mathbf{H}}=\mathrm{d}(\mathbf{H} \circ \boldsymbol{\Theta})=\mathbf{H} \circ \mathrm{d} \boldsymbol{\Theta}$ and $\mathrm{d}\left(\widehat{\mathbf{H}}^{*}\right)=\mathbf{H}^{*} \circ \mathrm{d}\left(\mathbf{\Theta}^{*}\right)$. Noting that $\left[\mathrm{d}\left(f^{\circ}(\mathbf{X})\right)\right]_{m, n}=\mathrm{d}\left(\left[f^{\circ}(\mathbf{X})\right]_{m, n}\right)=\mathrm{d}\left(f\left([\mathbf{X}]_{m, n}\right)\right)$, we obtain

$$
\begin{aligned}
\mathrm{d} \log \operatorname{det}\left(\left(\rho / M_{\mathrm{T}}\right) \widehat{\mathbf{Y}}\right) & =\log (e) \operatorname{Tr}\left(\widehat{\mathbf{Y}}^{-1}\left(\frac{1}{j} \widehat{\mathbf{H}}(\widehat{\mathbf{H}} \circ \mathrm{d} \boldsymbol{\Phi})^{H}-\frac{1}{j}(\widehat{\mathbf{H}} \circ \mathrm{d} \boldsymbol{\Phi}) \widehat{\mathbf{H}}^{H}\right)\right) \\
& =\log (e) \operatorname{Tr}\left(\widehat{\mathbf{Y}}^{-1}\left(\frac{1}{j} \widehat{\mathbf{H}}(\widehat{\mathbf{H}} \circ \mathrm{d} \boldsymbol{\Phi})^{H}-\frac{1}{j}\left(\widehat{\mathbf{H}}^{*}(\widehat{\mathbf{H}} \circ \mathrm{d} \boldsymbol{\Phi})^{H}\right)\right)\right) \\
& =2 \log (e) \operatorname{Im}\left(\operatorname{Tr}\left(\widehat{\mathbf{Y}}^{-1} \widehat{\mathbf{H}}(\widehat{\mathbf{H}} \circ \mathrm{d} \boldsymbol{\Phi})^{H}\right)\right) \\
& =2 \log (e) \operatorname{Im}\left(\operatorname{Tr}\left(\left(\widehat{\mathbf{Y}}^{-1} \widehat{\mathbf{H}}\right)^{T}\left(\widehat{\mathbf{H}}^{*} \circ \mathrm{d} \boldsymbol{\Phi}\right)\right)\right) .
\end{aligned}
$$

It remains to turn (31) into the form $\mathrm{d} \hat{I}=\mathrm{A} \operatorname{vec}(\mathrm{d} \boldsymbol{\Phi})$. This can be done by first showing that

$$
\operatorname{Tr}(\mathbf{A}(\mathbf{B} \circ \mathbf{C}))=\left(\operatorname{vec}\left(\mathbf{A}^{T} \circ \mathbf{B}\right)\right)^{T} \operatorname{vec}(\mathbf{C})
$$

and then applying (32) to (31). In order to prove (32), we start by noting that with $\operatorname{Tr}\left(\mathbf{A}^{T} \mathbf{B}\right)$ 
$=\operatorname{vec}(\mathbf{A})^{T} \operatorname{vec}(\mathbf{B})[12$, Sec. 2.4, Eq. (4)], we have

$$
\operatorname{Tr}(\mathbf{A}(\mathbf{B} \circ \mathbf{C}))=\left(\operatorname{vec}\left(\mathbf{A}^{T}\right)\right)^{T} \operatorname{vec}(\mathbf{B} \circ \mathbf{C})
$$

which upon application of

$$
\operatorname{vec}(\mathbf{A} \circ \mathbf{B})=\operatorname{divec}(\mathbf{A}) \operatorname{vec}(\mathbf{B})
$$

yields the desired result. Finally, applying (32) to (31), we obtain

$$
\mathrm{d} \log \operatorname{det}\left(\left(\rho / M_{\mathrm{T}}\right) \widehat{\mathbf{Y}}\right)=2 \log (e) \operatorname{Im}\left(\left(\operatorname{vec}\left(\left(\mathbf{Y}^{-1} \mathbf{H}\right) \circ \mathbf{H}^{*}\right)\right)^{T}\right) \operatorname{vec}(\mathrm{d} \boldsymbol{\Phi})
$$

which proves (28).

Computing the Hessian matrix: We start by noting that

$$
\mathrm{d}^{2} \log \operatorname{det}\left(\left(\rho / M_{\mathrm{T}}\right) \widehat{\mathbf{Y}}\right)=\log (e) \mathrm{d} \operatorname{Tr}\left(\widehat{\mathbf{Y}}^{-1} \mathrm{~d} \widehat{\mathbf{Y}}\right)=\log (e) \operatorname{Tr}\left(\widehat{\mathbf{Y}}^{-1}\left(\mathrm{~d}^{2} \widehat{\mathbf{Y}}\right)-\left(\widehat{\mathbf{Y}}^{-1} \mathrm{~d} \widehat{\mathbf{Y}}\right)^{2}\right)
$$

where we used $d \operatorname{Tr}(\mathbf{A})=\operatorname{Tr}(\mathrm{d} \mathbf{A})\left[12\right.$, Sec. 8.2, Eq. (20)] and $\mathrm{d}\left(\mathbf{A}^{-1}\right)=-\mathbf{A}^{-1}(\mathrm{~d} \mathbf{A}) \mathbf{A}^{-1}[12$, Sec. 8.4, Eq. (1)] along with the product rule. In order to keep the following exposition simple, we set $\mathrm{d} \widehat{\mathbf{H}}=j \widehat{\mathbf{H}} \circ \mathrm{d} \Phi=j \dot{\mathbf{H}}$ so that $\mathrm{d} \widehat{\mathbf{Y}}=\mathrm{d}\left(\widehat{\mathbf{H}} \widehat{\mathbf{H}}^{H}\right)=j \dot{\mathbf{H}} \widehat{\mathbf{H}}^{H}-j \widehat{\mathbf{H}} \dot{\mathbf{H}}^{H}$. Expanding the second term on the right-hand side (RHS) of (34) through similar manipulations as in the derivation of the Jacobian matrix, and using $\left(\widehat{\mathbf{Y}}^{-1}\right)^{T}=\left(\widehat{\mathbf{Y}}^{-1}\right)^{*}$, we get

$$
\begin{aligned}
-\operatorname{Tr}\left(\left(\widehat{\mathbf{Y}}^{-1} \mathrm{~d} \widehat{\mathbf{Y}}\right)^{2}\right) \\
\quad=\operatorname{Tr}\left(\widehat{\mathbf{Y}}^{-1}\left(\dot{\mathbf{H}} \widehat{\mathbf{H}}^{H}-\widehat{\mathbf{H}} \dot{\mathbf{H}}^{H}\right) \widehat{\mathbf{Y}}^{-1}\left(\dot{\mathbf{H}} \widehat{\mathbf{H}}^{H}-\widehat{\mathbf{H}} \dot{\mathbf{H}}^{H}\right)\right) \\
\quad=\operatorname{Tr}\left(\widehat{\mathbf{Y}}^{-1} \dot{\mathbf{H}} \widehat{\mathbf{H}}^{H} \widehat{\mathbf{Y}}^{-1} \dot{\mathbf{H}} \widehat{\mathbf{H}}^{H}\right)-\operatorname{Tr}\left(\widehat{\mathbf{Y}}-1 \dot{\mathbf{H}} \widehat{\mathbf{H}}^{H} \widehat{\mathbf{Y}}^{-1} \widehat{\mathbf{H}} \dot{\mathbf{H}}^{H}\right) \\
\quad+\operatorname{Tr}\left(\dot{\mathbf{H}}^{*} \widehat{\mathbf{H}}^{T}\left(\widehat{\mathbf{Y}}^{-1}\right)^{*} \dot{\mathbf{H}}^{*} \widehat{\mathbf{H}}^{T}\left(\widehat{\mathbf{Y}}^{-1}\right)^{*}\right)-\operatorname{Tr}\left(\widehat{\mathbf{H}}^{*} \dot{\mathbf{H}}^{T}\left(\widehat{\mathbf{Y}}^{-1}\right)^{*} \dot{\mathbf{H}}^{*} \widehat{\mathbf{H}}^{T}\left(\widehat{\mathbf{Y}}^{-1}\right)^{*}\right) \\
\quad=\operatorname{Tr}\left(\widehat{\mathbf{Y}}^{-1} \dot{\mathbf{H}} \widehat{\mathbf{H}}^{H} \widehat{\mathbf{Y}}^{-1} \dot{\mathbf{H}} \widehat{\mathbf{H}}^{H}\right)-\operatorname{Tr}\left(\widehat{\mathbf{Y}}^{-1} \dot{\mathbf{H}} \widehat{\mathbf{H}}^{H} \widehat{\mathbf{Y}}^{-1} \widehat{\mathbf{H}} \dot{\mathbf{H}}^{H}\right)
\end{aligned}
$$




$$
\begin{aligned}
& +\operatorname{Tr}\left(\left(\widehat{\mathbf{Y}}^{-1}\right)^{*} \dot{\mathbf{H}}^{*} \widehat{\mathbf{H}}^{T}\left(\widehat{\mathbf{Y}}^{-1}\right)^{*} \dot{\mathbf{H}}^{*} \widehat{\mathbf{H}}^{T}\right)-\operatorname{Tr}\left(\left(\widehat{\mathbf{Y}}^{-1}\right)^{*} \dot{\mathbf{H}}^{*} \widehat{\mathbf{H}}^{T}\left(\widehat{\mathbf{Y}}^{-1}\right)^{*} \widehat{\mathbf{H}}^{*} \dot{\mathbf{H}}^{T}\right) \\
& =2 \operatorname{Re}\left(\operatorname{Tr}\left(\widehat{\mathbf{Y}}^{-1} \dot{\mathbf{H}} \widehat{\mathbf{H}}^{H} \widehat{\mathbf{Y}}^{-1} \dot{\mathbf{H}} \widehat{\mathbf{H}}^{H}\right)\right)-2 \operatorname{Re}\left(\operatorname{Tr}\left(\widehat{\mathbf{Y}}^{-1} \dot{\mathbf{H}} \widehat{\mathbf{H}}^{H} \widehat{\mathbf{Y}}^{-1} \widehat{\mathbf{H}} \dot{\mathbf{H}}^{H}\right)\right) \\
& =2 \operatorname{Re}\left(\operatorname{Tr}\left(\widehat{\mathbf{H}}^{H} \widehat{\mathbf{Y}}^{-1} \dot{\mathbf{H}} \widehat{\mathbf{H}}^{H} \widehat{\mathbf{Y}}^{-1} \dot{\mathbf{H}}\right)\right)-2 \operatorname{Re}\left(\operatorname{Tr}\left(\left(\widehat{\mathbf{Y}}^{-1}\right)^{T} \dot{\mathbf{H}}^{*} \widehat{\mathbf{H}}^{T}\left(\widehat{\mathbf{Y}}^{-1}\right)^{*} \widehat{\mathbf{H}}^{*} \dot{\mathbf{H}}^{T}\right)\right) .
\end{aligned}
$$

Next, applying [12, Ch. 2, Th. 3]

$$
\operatorname{Tr}(\mathbf{A B C D})=\left(\operatorname{vec}\left(\mathbf{D}^{T}\right)\right)^{T}\left(\mathbf{C}^{T} \otimes \mathbf{A}\right) \operatorname{vec}(\mathbf{B})=(\operatorname{vec}(\mathbf{D}))^{T}\left(\mathbf{A} \otimes \mathbf{C}^{T}\right) \operatorname{vec}\left(\mathbf{B}^{T}\right)
$$

with $\mathbf{A}=\widehat{\mathbf{H}}^{H} \widehat{\mathbf{Y}}^{-1}, \mathbf{B}=\dot{\mathbf{H}}, \mathbf{C}=\widehat{\mathbf{H}}^{H} \widehat{\mathbf{Y}}^{-1}$, and $\mathbf{D}=\dot{\mathbf{H}}$ to the first term on the RHS of (35) and with $\mathbf{A}=\left(\widehat{\mathbf{Y}}^{-1}\right)^{T}, \mathbf{B}=\dot{\mathbf{H}}^{*}, \mathbf{C}=\widehat{\mathbf{H}}^{T}\left(\widehat{\mathbf{Y}}^{-1}\right)^{*} \widehat{\mathbf{H}}^{*}$, and $\mathbf{D}=\dot{\mathbf{H}}^{T}$ to the second term on the RHS of (35), we obtain

$$
\begin{aligned}
-\operatorname{Tr}\left(\left(\widehat{\mathbf{Y}}^{-1} \mathrm{~d} \widehat{\mathbf{Y}}\right)^{2}\right) & =2 \operatorname{Re}\left(\left(\operatorname{vec}\left(\dot{\mathbf{H}}^{T}\right)\right)^{T} \widehat{\mathbf{R}}_{1} \operatorname{vec}(\dot{\mathbf{H}})\right)-2 \operatorname{Re}\left(\left(\operatorname{vec}\left(\dot{\mathbf{H}}^{T}\right)\right)^{T} \widehat{\mathbf{R}}_{2} \operatorname{vec}\left(\dot{\mathbf{H}}^{H}\right)\right) \\
& \stackrel{(a)}{=} 2\left(\operatorname{vec}\left((\mathrm{d} \boldsymbol{\Phi})^{T}\right)\right)^{T} \operatorname{Re}\left(\operatorname{divec}\left(\widehat{\mathbf{H}}^{T}\right) \widehat{\mathbf{R}}_{1} \operatorname{divec}(\widehat{\mathbf{H}})\right) \operatorname{vec}(\mathrm{d} \boldsymbol{\Phi}) \\
& -2\left(\operatorname{vec}\left((\mathrm{d} \boldsymbol{\Phi})^{T}\right)\right)^{T} \operatorname{Re}\left(\operatorname{divec}\left(\widehat{\mathbf{H}}^{T}\right) \widehat{\mathbf{R}}_{2} \operatorname{divec}\left(\widehat{\mathbf{H}}^{H}\right)\right) \operatorname{vec}\left((\mathrm{d} \boldsymbol{\Phi})^{T}\right)
\end{aligned}
$$

where we set $\widehat{\mathbf{R}}_{1}=\left(\widehat{\mathbf{H}}^{H} \widehat{\mathbf{Y}}^{-1}\right)^{T} \otimes \widehat{\mathbf{H}}^{H} \widehat{\mathbf{Y}}^{-1}=\left(\widehat{\mathbf{Y}}^{-1} \otimes \widehat{\mathbf{H}}^{*}\right)^{T}\left(\widehat{\mathbf{H}}^{*} \otimes \widehat{\mathbf{Y}}^{-1}\right)$ and $\widehat{\mathbf{R}}_{2}=\left(\widehat{\mathbf{Y}}^{-1}\right)^{T} \otimes$ $\widehat{\mathbf{H}}^{H} \widehat{\mathbf{Y}}^{-1} \widehat{\mathbf{H}}$, and we used $\dot{\mathbf{H}}=\widehat{\mathbf{H}} \circ \mathrm{d} \boldsymbol{\Phi}$ and (33) in (a). Next, we need to rewrite (37) in terms of $(\operatorname{vec}(\mathrm{d} \boldsymbol{\Phi}))^{T}$ and $\operatorname{vec}(\mathrm{d} \boldsymbol{\Phi})$ only, which requires getting rid of the terms $(\mathrm{d} \boldsymbol{\Phi})^{T}$ inside the $(\operatorname{vec}(\cdot))^{T}$. Upon applying (1) in (37), we obtain

$$
\begin{aligned}
-\operatorname{Tr}\left(\left(\widehat{\mathbf{Y}}^{-1} \mathrm{~d} \widehat{\mathbf{Y}}\right)^{2}\right) & =2(\operatorname{vec}(\mathrm{d} \boldsymbol{\Phi}))^{T} \mathbf{K}_{\left(M_{\mathrm{R}}, M_{\mathrm{T}}\right)}^{T} \operatorname{Re}\left(\operatorname{divec}\left(\widehat{\mathbf{H}}^{T}\right) \widehat{\mathbf{R}}_{1} \operatorname{divec}(\widehat{\mathbf{H}})\right) \operatorname{vec}(\mathrm{d} \boldsymbol{\Phi}) \\
& -2(\operatorname{vec}(\mathrm{d} \boldsymbol{\Phi}))^{T} \mathbf{K}_{\left(M_{\mathrm{R}}, M_{\mathrm{T}}\right)}^{T} \operatorname{Re}\left(\operatorname{divec}\left(\widehat{\mathbf{H}}^{T}\right) \widehat{\mathbf{R}}_{2} \operatorname{divec}\left(\widehat{\mathbf{H}}^{H}\right)\right) \mathbf{K}_{\left(M_{\mathrm{R}}, M_{\mathrm{T}}\right)} \operatorname{vec}(\mathrm{d} \boldsymbol{\Phi})
\end{aligned}
$$

which using $\mathbf{K}_{(m, n)}^{T}=\mathbf{K}_{(n, m)}$ [12, Sec. 3.7, Eq. (2)] and

$$
(\operatorname{vec}(\mathbf{A}))^{T} \mathbf{K}_{(n, m)} \operatorname{divec}(\mathbf{B})=(\operatorname{vec}(\mathbf{A}))^{T} \operatorname{divec}\left(\mathbf{B}^{T}\right) \mathbf{K}_{(n, m)}
$$


results in

$$
\begin{aligned}
& -\operatorname{Tr}\left(\left(\widehat{\mathbf{Y}}^{-1} \mathrm{~d} \widehat{\mathbf{Y}}\right)^{2}\right) \\
& \quad=2(\operatorname{vec}(\mathrm{d} \boldsymbol{\Phi}))^{T} \operatorname{Re}\left(\operatorname{divec}(\widehat{\mathbf{H}}) \mathbf{K}_{\left(M_{\mathrm{T}}, M_{\mathrm{R}}\right)} \widehat{\mathbf{R}}_{1} \operatorname{divec}(\widehat{\mathbf{H}})\right) \operatorname{vec}(\mathrm{d} \boldsymbol{\Phi}) \\
& \quad-2(\operatorname{vec}(\mathrm{d} \boldsymbol{\Phi}))^{T} \operatorname{Re}\left(\operatorname{divec}(\widehat{\mathbf{H}}) \mathbf{K}_{\left(M_{\mathrm{T}}, M_{\mathrm{R}}\right)} \widehat{\mathbf{R}}_{2} \mathbf{K}_{\left(M_{\mathrm{R}}, M_{\mathrm{T}}\right)} \operatorname{divec}\left(\widehat{\mathbf{H}}^{*}\right)\right) \operatorname{vec}(\mathrm{d} \boldsymbol{\Phi}) .
\end{aligned}
$$

The validity of (38) can easily be seen by noting that $\operatorname{divec}(\mathbf{B}) \mathbf{K}_{(m, n)} \operatorname{vec}(\mathbf{A})$ = $\operatorname{divec}(\mathbf{B}) \operatorname{vec}\left(\mathbf{A}^{T}\right)=\operatorname{vec}\left(\mathbf{B} \circ \mathbf{A}^{T}\right)=\operatorname{vec}\left(\left(\mathbf{B}^{T} \circ \mathbf{A}\right)^{T}\right)=\mathbf{K}_{(m, n)} \operatorname{vec}\left(\mathbf{B}^{T} \circ \mathbf{A}\right)=$ $\mathbf{K}_{(m, n)} \operatorname{divec}\left(\mathbf{B}^{T}\right) \operatorname{vec}(\mathbf{A})$.

Finally, employing [12, Sec. 3.7, Eq. (5)]

$$
\mathbf{K}_{(p, m)}(\mathbf{A} \otimes \mathbf{B}) \mathbf{K}_{(n, q)}=\mathbf{B} \otimes \mathbf{A}
$$

to the $m \times n$ matrix $\mathbf{A}$ and the $p \times q$ matrix $\mathbf{B}$, we can simplify the second term on the RHS of (39) to obtain

$$
\begin{array}{r}
-\operatorname{Tr}\left(\left(\widehat{\mathbf{Y}}^{-1} \mathrm{~d} \widehat{\mathbf{Y}}\right)^{2}\right) \\
=2(\operatorname{vec}(\mathrm{d} \boldsymbol{\Phi}))^{T} \operatorname{Re}\left(\operatorname{divec}(\widehat{\mathbf{H}}) \mathbf{K}_{\left(M_{\mathrm{T}}, M_{\mathrm{R}}\right)}\left(\left(\widehat{\mathbf{H}}^{H} \widehat{\mathbf{Y}}^{-1}\right)^{T} \otimes\left(\widehat{\mathbf{H}}^{H} \widehat{\mathbf{Y}}^{-1}\right)\right) \operatorname{divec}(\widehat{\mathbf{H}})\right. \\
\left.\quad-\operatorname{divec}(\widehat{\mathbf{H}})\left(\left(\widehat{\mathbf{H}}^{H} \widehat{\mathbf{Y}}^{-1} \widehat{\mathbf{H}}\right) \otimes\left(\widehat{\mathbf{Y}}^{-1}\right)^{T}\right) \operatorname{divec}\left(\widehat{\mathbf{H}}^{*}\right)\right) \operatorname{vec}(\mathrm{d} \boldsymbol{\Phi}) .
\end{array}
$$

It remains to turn the first term on the RHS of (34) into the form of the RHS of (30). To this end, we start by noting that, using Wirtinger calculus,

$$
\mathrm{d}^{2} \widehat{\mathbf{Y}}=\mathrm{d} \mathrm{d}\left(\widehat{\mathbf{H}} \widehat{\mathbf{H}}^{H}\right)=\mathrm{d}\left(j \dot{\mathbf{H}} \widehat{\mathbf{H}}^{H}-j \widehat{\mathbf{H}} \dot{\mathbf{H}}^{H}\right)=-\ddot{\mathbf{H}} \widehat{\mathbf{H}}^{H}+\dot{\mathbf{H}} \dot{\mathbf{H}}^{H}+\dot{\mathbf{H}} \dot{\mathbf{H}}^{H}-\widehat{\mathbf{H}} \ddot{\mathbf{H}}^{H}
$$

where we set $\mathrm{d}^{2} \widehat{\mathbf{H}}=-\widehat{\mathbf{H}} \circ \mathrm{d} \boldsymbol{\Phi} \circ \mathrm{d} \boldsymbol{\Phi}=-\ddot{\mathbf{H}}$. Next, inserting (42) into the first term on the RHS of (34), we get

$$
\operatorname{Tr}\left(\widehat{\mathbf{Y}}^{-1} \mathrm{~d}^{2} \widehat{\mathbf{Y}}\right)
$$




$$
\begin{aligned}
& =2 \operatorname{Tr}\left(\widehat{\mathbf{Y}}^{-1} \dot{\mathbf{H}} \dot{\mathbf{H}}^{H}\right)-\operatorname{Tr}\left(\widehat{\mathbf{Y}}^{-1} \ddot{\mathbf{H}} \widehat{\mathbf{H}}^{H}\right)-\operatorname{Tr}\left(\widehat{\mathbf{Y}}^{-1} \widehat{\mathbf{H}} \ddot{\mathbf{H}}^{H}\right) \\
& =2 \operatorname{Tr}\left(\widehat{\mathbf{Y}}^{-1} \dot{\mathbf{H}} \dot{\mathbf{H}}^{H}\right)-\operatorname{Tr}\left(\widehat{\mathbf{Y}}^{-1} \ddot{\mathbf{H}} \widehat{\mathbf{H}}^{H}\right)-\operatorname{Tr}\left(\left(\widehat{\mathbf{Y}}^{-1}\right)^{*} \ddot{\mathbf{H}}^{*} \widehat{\mathbf{H}}^{T}\right) \\
& =2 \operatorname{Tr}\left(\widehat{\mathbf{Y}}^{-1} \dot{\mathbf{H}} \dot{\mathbf{H}}^{H}-\operatorname{Re}\left(\widehat{\mathbf{Y}}^{-1} \ddot{\mathbf{H}} \widehat{\mathbf{H}}^{H}\right)\right) \\
& =2 \operatorname{Tr}\left(\widehat{\mathbf{Y}}^{-1} \dot{\mathbf{H}} \mathbf{I}_{M_{\mathrm{T}}} \dot{\mathbf{H}}^{H}\right)-2 \operatorname{Re}\left(\operatorname{Tr}\left(\widehat{\mathbf{H}}^{H} \widehat{\mathbf{Y}}^{-1} \ddot{\mathbf{H}}\right)\right) \\
& \stackrel{(a)}{=} 2\left(\operatorname{vec}\left(\dot{\mathbf{H}}^{T}\right)\right)^{T}\left(\left(\widehat{\mathbf{Y}}^{-1}\right)^{T} \otimes \mathbf{I}_{M_{\mathrm{T}}}\right) \operatorname{vec}\left(\dot{\mathbf{H}}^{H}\right)-2 \operatorname{Re}\left(\left(\operatorname{vec}\left(\left(\widehat{\mathbf{H}}^{H} \widehat{\mathbf{Y}}^{-1}\right)^{T}\right)\right)^{T} \operatorname{vec}(\ddot{\mathbf{H}})\right) \\
& \stackrel{\text { (b) }}{=} 2(\operatorname{vec}(\mathrm{d} \boldsymbol{\Phi}))^{T} \mathbf{K}_{\left(M_{\mathrm{T}}, M_{\mathrm{R}}\right)} \operatorname{divec}\left(\widehat{\mathbf{H}}^{T}\right)\left(\left(\widehat{\mathbf{Y}}^{-1}\right)^{T} \otimes \mathbf{I}_{M_{\mathrm{T}}}\right) \operatorname{divec}\left(\widehat{\mathbf{H}}^{H}\right) \mathbf{K}_{\left(M_{\mathrm{R}}, M_{\mathrm{T}}\right)} \operatorname{vec}(\mathrm{d} \boldsymbol{\Phi}) \\
& -2 \operatorname{Re}\left(\left(\operatorname{vec}\left(\left(\widehat{\mathbf{H}}^{H} \widehat{\mathbf{Y}}^{-1}\right)^{T}\right)\right)^{T} \operatorname{divec}(\widehat{\mathbf{H}}) \operatorname{divec}(\mathrm{d} \boldsymbol{\Phi}) \operatorname{vec}(\mathrm{d} \mathbf{\Phi})\right) \\
& \stackrel{\text { (c) }}{=} 2(\operatorname{vec}(\mathrm{d} \boldsymbol{\Phi}))^{T} \operatorname{divec}(\widehat{\mathbf{H}})\left(\mathbf{I}_{M_{\mathrm{T}}} \otimes\left(\widehat{\mathbf{Y}}^{-1}\right)^{T}\right) \operatorname{divec}\left(\widehat{\mathbf{H}}^{*}\right) \operatorname{vec}(\mathrm{d} \boldsymbol{\Phi}) \\
& -2(\operatorname{vec}(\mathrm{d} \boldsymbol{\Phi}))^{T} \operatorname{Re}\left(\operatorname{divec}\left(\left(\widehat{\mathbf{H}}^{H} \widehat{\mathbf{Y}}^{-1}\right)^{T} \circ \widehat{\mathbf{H}}\right)\right) \operatorname{vec}(\mathrm{d} \boldsymbol{\Phi})
\end{aligned}
$$

where (a) results from applying (36) with $\mathbf{A}=\widehat{\mathbf{Y}}^{-1}, \mathbf{B}=\dot{\mathbf{H}}, \mathbf{C}=\mathbf{I}_{M_{\mathrm{T}}}$, and $\mathbf{D}=\dot{\mathbf{H}}^{H}$ to the first term, transposing the result, and, as before, applying $\operatorname{Tr}\left(\mathbf{A}^{T} \mathbf{B}\right)=\operatorname{vec}(\mathbf{A})^{T} \operatorname{vec}(\mathbf{B})$ with $\mathbf{A}^{T}=\widehat{\mathbf{H}}^{H} \widehat{\mathbf{Y}}^{-1}$ and $\mathbf{B}=\ddot{\mathbf{H}}$ to the second term. Step (b) is a consequence of applying (33), the commutation relation (1), and $\mathbf{K}_{(m, n)}^{T}=\mathbf{K}_{(n, m)}$. To obtain (c), we used (38) and (40) for the first term and

$$
\begin{aligned}
\left(\operatorname{vec}\left(\left(\widehat{\mathbf{H}}^{H} \widehat{\mathbf{Y}}^{-1}\right)^{T}\right)\right)^{T} \operatorname{divec}(\widehat{\mathbf{H}}) \operatorname{divec}(\mathrm{d} \boldsymbol{\Phi}) & =\left(\operatorname{vec}\left(\left(\widehat{\mathbf{H}}^{H} \widehat{\mathbf{Y}}^{-1}\right)^{T}\right)\right)^{T} \operatorname{divec}(\mathrm{d} \boldsymbol{\Phi}) \operatorname{divec}(\widehat{\mathbf{H}}) \\
& =(\operatorname{vec}(\mathrm{d} \boldsymbol{\Phi}))^{T} \operatorname{divec}\left(\left(\widehat{\mathbf{H}}^{H} \widehat{\mathbf{Y}}^{-1}\right)^{T} \circ \widehat{\mathbf{H}}\right)
\end{aligned}
$$

for the second term. The final result follows by identifying (45) and (41) with the RHS of (30), noting that $\widehat{\mathbf{H}}=\mathbf{H}$ for $\boldsymbol{\Phi}=\mathbf{0}$, and applying the second identification theorem. All the terms, except for the first term in (45), can be verified to be real-symmetrid 8 so that $(1 / 2)\left(\mathbf{B}+\mathbf{B}^{T}\right)=\mathbf{B}$. The first term in (45) is Hermitian and hence $(1 / 2)\left(\mathbf{B}+\mathbf{B}^{T}\right)=\operatorname{Re}(\mathbf{B})$.

\footnotetext{
${ }^{8} \mathrm{~A}$ matrix $\mathbf{X}$ is said to be real-symmetric if $\mathbf{X}=\mathbf{X}^{T}$.
} 


\section{B. Approximations for First and Second Moment of MI}

Even though the physical channel is deterministic, the effective channel $\widehat{\mathbf{H}}=\mathbf{H} \circ \exp ^{\circ}(j \boldsymbol{\Phi})$ will be random due to phase noise. We shall next compute approximations of the effective channel's ergodic capacity $\widehat{C}=\mathbb{E}\{\hat{I}\}$ and of $\operatorname{Var}\{\hat{I}\}$ based on the second-order Taylor series expansion (27). For an explanation of the operational significance of $\operatorname{Var}\{\hat{I}\}$ the reader is referred to Section VI and to [33]. Unlike the results in Section VI, which are restricted to the (extreme, but not necessarily untypical) case of fully uncorrelated phase noise, we will allow a general phase noise covariance matrix, i.e., $\boldsymbol{\Phi} \stackrel{\mathrm{d}}{=} \mathcal{N}\left(\mathbf{0}, \boldsymbol{\Sigma}_{\varphi}\right)$. Noting that $\mathcal{J}_{\hat{I}}(\mathbf{0}) \operatorname{vec}(\boldsymbol{\Phi})$ is zero-mean Gaussian and $\mathbf{Q}_{\hat{I}}=\mathbf{Q}_{\hat{I}}(\operatorname{vec}(\boldsymbol{\Phi}))=(\operatorname{vec}(\boldsymbol{\Phi}))^{T} \mathcal{H}_{\hat{I}}(\mathbf{0}) \operatorname{vec}(\boldsymbol{\Phi})$ is a quadratic form in real-valued Gaussian RVs with distribution [41, Eq. (4.1.1)]

$$
\mathbf{Q}_{\hat{I}} \stackrel{\mathrm{d}}{=} \sum_{i=1}^{M_{\mathrm{T}} M_{\mathrm{R}}} \lambda_{i}\left(\boldsymbol{\Sigma}_{\varphi}^{1 / 2} \mathcal{H}_{\hat{I}}(\mathbf{0}) \boldsymbol{\Sigma}_{\varphi}^{1 / 2}\right) X_{i}
$$

where the $X_{i} \stackrel{\mathrm{d}}{=} \chi_{1,1}^{2}\left(i=1,2, \ldots, M_{\mathrm{T}} M_{\mathrm{R}}\right)$ are statistically independent, straightforward manipulations reveal that

$$
\mathbb{E}\{\tilde{I}(\boldsymbol{\Phi})\}=\hat{I}(\mathbf{0})+\frac{1}{2} \operatorname{Tr}\left(\mathcal{H}_{\hat{I}}(\mathbf{0}) \boldsymbol{\Sigma}_{\varphi}\right)
$$

and

$$
\operatorname{Var}\{\tilde{I}(\mathbf{\Phi})\}=\mathcal{J}_{\hat{I}}(\mathbf{0}) \boldsymbol{\Sigma}_{\varphi} \mathcal{J}_{\hat{I}}^{T}(\mathbf{0})+\frac{1}{2} \operatorname{Tr}\left(\left(\mathcal{H}_{\hat{I}}(\mathbf{0}) \boldsymbol{\Sigma}_{\varphi}\right)^{2}\right)
$$

Inserting (28) and (29) into (46) and (47), we have analytic approximations of the ergodic capacity and the variance of the MI of the effective channel as a function of the physical channel and of the phase noise covariance matrix $\Sigma_{\varphi}$. The following numerical results demonstrate that these approximations tend to be quite accurate for full-rank physical channels but rather loose for rank-deficient physical channels.

Numerical results: In Fig. 4, we plot $\mathbb{E}\{\tilde{I}(\boldsymbol{\Phi})\}$ and $(\operatorname{Var}\{\tilde{I}(\boldsymbol{\Phi})\})^{1 / 2}$ in (46) and (47), re- 
spectively, versus the exact values $\mathbb{E}\{\hat{I}(\boldsymbol{\Phi})\}$ and $(\operatorname{Var}\{\hat{I}(\boldsymbol{\Phi})\})^{1 / 2}$ obtained by Monte Carlo simulation over 10000 phase noise samples with $3.5^{\circ} \mathrm{rms}$ fully uncorrelated phase noise. Each point in the figures represents a pair of exact and approximate MI first and second moments for one of 2000 realizations of an i.i.d. physical channel with $M_{\mathrm{T}}=M_{\mathrm{R}}=4$ at $\rho=30 \mathrm{~dB}$. We observe that the deviation of the first moment of MI can be positive or negative and is essentially independent of the exact value of the first moment. The deviation of the second moment is predominantly positive, and the accuracy shows a strong dependence on the exact value of the second moment. Quantitatively speaking, the deviation of the estimates $\mathbb{E}\{\tilde{I}(\boldsymbol{\Phi})\}$ and $(\operatorname{Var}\{\tilde{I}(\boldsymbol{\Phi})\})^{1 / 2}$ is significant for about $5 \%$ of the i.i.d. channel realizations.

\section{Taylor Series Expansion of the Channel's Eigenvalues}

To obtain more insight into the quality-of-fit of the second-order Taylor series approximation of MI, we show in Fig. [5] the cdfs of the exact MI and the MI obtained through the approximation (27). In all cases, Monte Carlo simulation at $\rho=30 \mathrm{~dB}$ with $3.5^{\circ} \mathrm{rms}$ fully uncorrelated phase noise was employed. As (deterministic) physical channels we have chosen balanced (i.e., all nonzero singular values of the channel matrix are equal) rank- $M 4 \times 4$ channels for $M=$ $1,2,3,4$ and an unbalanced full-rank channel. All physical channels were normalized to satisfy $\|\mathbf{H}\|_{\mathrm{F}}^{2}=M_{\mathrm{T}} M_{\mathrm{R}}=16$. The figure shows that the Taylor series approximation is very loose for rank-1 and rank-2 physical channels, acceptable for the rank-3 physical channel, and very accurate for the two full-rank physical channels. This example shows that the second-order Taylor series expansion of MI tends to yield poor approximations for low-rank or, more generally, poorly balanced physical channels, i.e., physical channels with large eigenvalue spread. An alternative approach for obtaining approximations of $\hat{I}$ is to compute a second-order Taylor series expansion of the unordered (but continuous w.r.t. $\Phi$ ) eigenvalues $\lambda_{\widehat{\mathbf{H}} \hat{\mathbf{H}}^{H}}^{(i)}(\Phi)$ where

$$
\hat{I}(\boldsymbol{\Phi})=\sum_{i=1}^{M_{\mathrm{R}}} \log \left(1+\frac{\rho}{M_{\mathrm{T}}} \lambda_{\widehat{\mathbf{H}}^{H}}^{(i)}(\boldsymbol{\Phi})\right) .
$$


Before briefly outlining how this can be done analytically, we show in Fig. 5[(b) the result of replacing (27) in Fig. 5[a) by the second-order Taylor series expansion of the eigenvalues $\lambda_{\widehat{\mathbf{H}} \widehat{\mathbf{H}}^{H}}^{(i)}(\boldsymbol{\Phi})$ used in the RHS of (48). It is clearly seen that the Taylor series expansion of the eigenvalues $\lambda_{\widehat{\mathbf{H}} \widehat{\mathbf{H}}^{H}}^{(i)}(\boldsymbol{\Phi})$ yields outstanding accuracy and significantly better results than the Taylor series expansion of MI directly. A few comments on how to obtain analytic expressions for the second-order Taylor series expansion of the $\lambda_{\widehat{\mathbf{H}} \widehat{\mathbf{H}}^{H}}^{(i)}(\boldsymbol{\Phi})$ are in order. For the sake of space and focus of the paper, we shall not present the details, but rather refer the interested reader to suitable references. In general, obtaining analytic expressions for the Taylor series of the $\lambda_{\widehat{\mathbf{H}} \widehat{\mathbf{H}}^{H}}^{(i)}(\boldsymbol{\Phi})$ is difficult and tedious; in fact, in general, more tedious than computing the Taylor series expansion of MI directly. While the case of a physical channel with nonrepeated eigenvalues can be treated with relative ease by employing $[12$, Ch. 8 , Ths. $7,8,10$, and 11], the general case of physical channels that have eigenvalues of multiplicity larger than 1 (e.g., multiple eigenvalues equal to zero in the case of rank-deficient physical channels) is significantly more involved. Results relevant in this context can be found in [12, Ch. 8, Sec. 12] and in [42]. The main difficulty in obtaining analytic expressions in the case of repeated eigenvalues is that the results depend on the eigenvectors of the channel.

\section{The RANK-1 PhysicAl CHANNEL}

As already mentioned in Section IV] the impact of phase errors is more pronounced for lowrank physical MIMO channels. In the following, we shall therefore analyze the extreme case of a rank-1 physical channel in detail. In practice, deterministic rank-1 channels occur in lineof-sight (LOS) scenarios with small angle-spread [2] (green-field like propagation conditions). Stochastic rank-1 MIMO channels are channels where the realization of the MIMO channel matrix has rank 1 w.p.1. A prominent member of this class of channels is the pin-hole [2] or key-hole [3], [10], [11] channel reflecting propagation conditions with significant scattering close to the transmitter and the receiver and at the same time long distances between transmitter 
and receiver. Finally, the following MIMO channel sounder "calibration procedure" provides a practical motivation for studying (and quantifying) the impact of phase errors on rank-1 physical channels. The main idea underlying this calibration procedure is based on the fact that connecting transmitter and receiver in a TDMS-based sounder by a cable results in a deterministic rank1 physical channel $\mathbf{H}=\alpha \mathbf{1}$, where $\alpha \in \mathbb{C}$ is the gain corresponding to the constant (across frequency) cable transfer function. The channel sounder then acquires samples of the effective channel matrix which contains channel coefficients that (after power normalization) have unit magnitude and a phase that varies due to phase errors created by the sounder. An inspection of the resulting eigenvalue histogram yields the number of significant eigenvalues and the corresponding eigenvalue distribution. Since the underlying physical channel has rank 1, it follows that any additional (w.r.t. the one resulting from the physical channel) significant modes in the effective channel must necessarily be due to phase errors (and/or potentially other imperfections in the measurement equipment). This "calibration measurement" can therefore loosely be interpreted as revealing the highest possible rank increase due to phase errors.

We shall see that, unlike the general case discussed in Sections $I V$ and $\square$ rank-1 physical channels allow to establish a number of insightful analytic results on the impact of phase errors on MI. Throughout this section, unless explicitly stated otherwise, the results are valid for a general (i.e., not necessarily separable) timing matrix $\mathbf{T}$. We consider channels given by $\mathbf{H}=\mathbf{g h}^{T}$ where the vectors $\mathbf{g}$ and $\mathbf{h}$ can be either deterministic or stochastic (and with entries that are not necessarily unit modulus).

Let us start with a simple basic result which will be needed later in this section.

Lemma 2: The Hadamard product of a rank-1 matrix $\mathbf{H}=\mathbf{g h}^{T}$ and an arbitrary matrix $\Theta$ can be written as a matrix product according to

$$
\left(\mathbf{g h} \mathbf{h}^{T}\right) \circ \boldsymbol{\Theta}=\operatorname{diag}(\mathbf{g}) \boldsymbol{\Theta} \operatorname{diag}(\mathbf{h}) .
$$


Proof: The elements of a rank-1 matrix $\mathbf{H}=\mathbf{g h}^{T}$ are given by $[\mathbf{H}]_{m, n}=[\mathbf{g}]_{m}[\mathbf{h}]_{n}$. Consequently, we have $[\mathbf{H} \circ \boldsymbol{\Theta}]_{m, n}=[\mathbf{g}]_{m}[\mathbf{h}]_{n}[\boldsymbol{\Theta}]_{m, n}$. On the other hand, it follows immediately that $[\operatorname{diag}(\mathbf{g}) \boldsymbol{\Theta} \operatorname{diag}(\mathbf{h})]_{m, n}=[\mathbf{g}]_{m}[\boldsymbol{\Theta}]_{m, n}[\mathbf{h}]_{n}$ which concludes the proof.

The following three Theorems state that a physical rank-1 channel subject to severe enough phase errors results in a full-rank effective channel.

Theorem 2: For a rank-1 physical channel $\mathbf{H}=\mathbf{g h}^{T}$ subject to phase errors with $\Theta$ in (20) having full rank w.p.1, we have

$$
\begin{aligned}
& \operatorname{det}\left(\widehat{\mathbf{H}} \widehat{\mathbf{H}}^{H}\right)=\left(\prod_{i=1}^{M_{\mathrm{R}}}\left|[\mathbf{g}]_{i}\right|^{2} \varepsilon_{i}\right) \operatorname{det}\left(\boldsymbol{\Theta}^{H}\right), \quad M_{\mathrm{R}} \leq M_{\mathrm{T}} \\
& \operatorname{det}\left(\widehat{\mathbf{H}}{ }^{H} \widehat{\mathbf{H}}\right)=\left(\prod_{i=1}^{M_{\mathrm{T}}}\left|[\mathbf{h}]_{i}\right|^{2} \nu_{i}\right) \operatorname{det}\left(\boldsymbol{\Theta}^{H} \boldsymbol{\Theta}\right), \quad M_{\mathrm{R}} \geq M_{\mathrm{T}}
\end{aligned}
$$

where

$$
\min _{i}\left|[\mathbf{h}]_{i}\right|^{2} \leq \varepsilon_{i} \leq \max _{i}\left|[\mathbf{h}]_{i}\right|^{2}, \quad \quad \min _{i}\left|[\mathbf{g}]_{i}\right|^{2} \leq \nu_{i} \leq \max _{i}\left|[\mathbf{g}]_{i}\right|^{2}
$$

Proof: We provide the proof for the case $M_{\mathrm{R}} \leq M_{\mathrm{T}}$ only. The proof for $M_{\mathrm{R}}>M_{\mathrm{T}}$ follows exactly the same line of reasoning. We start by noting that Lemma 2 implies

$$
\begin{aligned}
\operatorname{det}\left(\widehat{\mathbf{H}} \widehat{\mathbf{H}}^{H}\right) & =\operatorname{det}\left(\operatorname{diag}(\mathbf{g}) \boldsymbol{\Theta} \operatorname{diag}(\mathbf{h})(\operatorname{diag}(\mathbf{h}))^{H} \boldsymbol{\Theta}^{H}(\operatorname{diag}(\mathbf{g}))^{H}\right) \\
& =\left(\prod_{i=1}^{M_{\mathrm{R}}}\left|[\mathbf{g}]_{i}\right|^{2}\right) \prod_{i=1}^{M_{\mathrm{R}}} \lambda_{i}\left(\boldsymbol{\Theta} \operatorname{diag}(\mathbf{h})(\operatorname{diag}(\mathbf{h}))^{H} \boldsymbol{\Theta}^{H}\right) \\
& \stackrel{(\text { a) }}{=}\left(\prod_{i=1}^{M_{\mathrm{R}}}\left|[\mathbf{g}]_{i}\right|^{2}\right) \prod_{i=1}^{M_{\mathrm{R}}} \lambda_{i}\left((\operatorname{diag}(\mathbf{h}))^{H} \boldsymbol{\Theta}^{H} \boldsymbol{\Theta} \operatorname{diag}(\mathbf{h})\right) \\
& \stackrel{(\mathbf{b})}{=}\left(\prod_{i=1}^{M_{\mathrm{R}}}\left|[\mathbf{g}]_{i}\right|^{2}\right) \prod_{i=1}^{M_{\mathrm{R}}} \varepsilon_{i} \lambda_{i}\left(\boldsymbol{\Theta} \Theta^{H}\right)
\end{aligned}
$$

where the second product on the RHS of (a) is taken over the $M_{\mathrm{R}}$ nonzero eigenvalues of $(\operatorname{diag}(\mathbf{h}))^{H} \boldsymbol{\Theta}^{H} \boldsymbol{\Theta} \operatorname{diag}(\mathbf{h})$ only (note that the $\lambda_{i}$ are ordered as defined in the Notations section) and (b) follows from a Corollary to Ostrowski's Theorem [43, Corollary 4.5.11]. 
Theorem 2 thus states that a physical rank-1 channel subject to phase noise such that $\Theta$ has full rank w.p.1 results in a full-rank effective MIMO channel (provided that $[\mathbf{g}]_{m} \neq 0, \forall m$, and $\left.[\mathbf{h}]_{n} \neq 0, \forall n\right)$. For a deterministic physical rank-1 channel, the resulting effective channel will be stochastic and will have full rank w.p.1. The condition of $\Theta$ having full rank w.p.1 may sound stringent. It turns out, however, that a full-rank phase noise covariance matrix $\operatorname{Cov}\{\boldsymbol{\Phi}\}$ is sufficient for $\Theta$ to have full rank w.p.1. This statement can be formalized as follows.

Lemma 3: A real Gaussian random matrix $\boldsymbol{\Phi} \in \mathbb{R}^{M_{\mathrm{R}} \times M_{\mathrm{T}}}$ where $\operatorname{vec}(\boldsymbol{\Phi}) \stackrel{\mathrm{d}}{=} \mathcal{N}(\mathbf{0}, \operatorname{Cov}\{\boldsymbol{\Phi}\})$ with $\operatorname{det}(\operatorname{Cov}\{\boldsymbol{\Phi}\})>0$, has full rank w.p.1. The matrix $\Theta=\exp ^{\circ}(j \Phi)$ has full rank w.p.1 as well.

Proof: We follow the direct proof of [44, Th. 2.3, p. 712], where it is shown that for an $M_{\mathrm{R}} \times M_{\mathrm{T}}$ random matrix $\mathrm{X}$ to be full rank w.p.1, it is sufficient to have the multivariate distribution of $\mathbf{X}$ be absolutely continuous w.r.t. $M_{\mathrm{R}} M_{\mathrm{T}}$-dimensional Lebesgue measure. This condition is trivially satisfied by $\boldsymbol{\Phi}$ with $\operatorname{vec}(\boldsymbol{\Phi}) \stackrel{\mathrm{d}}{=} \mathcal{N}(\mathbf{0}, \operatorname{Cov}\{\boldsymbol{\Phi}\})$ and $\operatorname{det}(\operatorname{Cov}\{\boldsymbol{\Phi}\})>0$ (see, e.g., [45, Sec. 4.7.2]).

The second part of the statement can be proved by using [46, Lemma 3], which states that $\boldsymbol{\Theta}$ has full rank if either $\operatorname{Re}(\boldsymbol{\Theta}), \operatorname{Im}(\boldsymbol{\Theta})$, or $\left[(\operatorname{Re}(\boldsymbol{\Theta}))^{T}(\operatorname{Im}(\boldsymbol{\Theta}))^{T}\right]^{T}$ has full rank. Direct computation reveals that the multivariate pdf of $(\operatorname{Re}(\boldsymbol{\Theta}))^{T}=\cos ^{\circ}(\boldsymbol{\Theta})$ is continuous and integrable (in the interval $[-1,1]^{M_{\mathrm{T}} M_{\mathrm{R}}}$ ) so that its multivariate cdf is absolutely continuous w.r.t. $M_{\mathrm{R}} M_{\mathrm{T}}$-dimensional Lebesgue measure. Hence, by the direct proof of [44, Th. 2.3], $\operatorname{Re}(\Theta)$ is full rank w.p.1, and from what was said before it follows that $\Theta$ has full rank w.p.1.

Besides what was stated in Theorem 2 above, relating properties of $\operatorname{Cov}\{\boldsymbol{\Phi}\}$ to properties of $\operatorname{det}\left(\Theta \Theta^{H}\right)$ seems difficult. For $M_{\mathrm{T}}=M_{\mathrm{R}}$, we can refine the result in Theorem 2 as follows.

Theorem 3: For a rank-1 physical channel $\mathbf{H}=\mathbf{g h}^{T}$ with $M_{\mathrm{T}}=M_{\mathrm{R}}=M$ subject to phase errors with $\Theta$ having full rank w.p.1, we have

$$
\operatorname{det}\left(\widehat{\mathbf{H}} \widehat{\mathbf{H}}^{H}\right)=\left(\prod_{i=1}^{M}\left|[\mathbf{h}]_{i}\right|^{2}\right)\left(\prod_{i=1}^{M}\left|[\mathbf{g}]_{i}\right|^{2}\right) \operatorname{det}\left(\boldsymbol{\Theta} \Theta^{H}\right) .
$$


Proof: The proof follows trivially using Lemma 2 and noting that

$$
\begin{aligned}
\operatorname{det}\left(\widehat{\mathbf{H}} \widehat{\mathbf{H}}^{H}\right) & =\operatorname{det}\left(\operatorname{diag}(\mathbf{g}) \boldsymbol{\Theta} \operatorname{diag}(\mathbf{h})(\operatorname{diag}(\mathbf{h}))^{H} \boldsymbol{\Theta}^{H}(\operatorname{diag}(\mathbf{g}))^{H}\right) \\
& =\operatorname{det}\left((\operatorname{diag}(\mathbf{g}))^{H} \operatorname{diag}(\mathbf{g})\right) \operatorname{det}\left(\boldsymbol{\Theta} \operatorname{diag}(\mathbf{h})(\operatorname{diag}(\mathbf{h}))^{H} \boldsymbol{\Theta}^{H}\right) \\
& =\operatorname{det}\left((\operatorname{diag}(\mathbf{g}))^{H} \operatorname{diag}(\mathbf{g})\right) \operatorname{det}\left(\operatorname{diag}(\mathbf{h})(\operatorname{diag}(\mathbf{h}))^{H}\right) \operatorname{det}\left(\boldsymbol{\Theta} \boldsymbol{\Theta}^{H}\right)
\end{aligned}
$$

which yields (49).

The following Theorem allows a more specific conclusion since it shows that for rank-1 channels $\mathbf{H}=\mathbf{g h}^{T}$ where $\mathbf{g}$ and $\mathbf{h}$ consist of unit-modulus entries (representative of LOS propagation [2]) the rank of the effective channel matrix is equal to the rank of $\Theta$. Moreover, the eigenvalues of the effective channel matrix (more specifically of $\widehat{\mathbf{H}} \widehat{\mathbf{H}}^{H}$ ) are equal to the eigenvalues of $\Theta \Theta^{H}$.

Theorem 4: For a rank-1 physical channel $\mathbf{H}=\mathbf{g h}^{T}$, where $\mathbf{g}$ and $\mathbf{h}$ are such that $\left|[\mathbf{g}]_{i}\right|=1$ $\left(i=1,2, \ldots, M_{\mathrm{R}}\right)$ and $\left|[\mathbf{h}]_{i}\right|=1\left(i=1,2, \ldots, M_{\mathrm{T}}\right)$, we have

$$
\begin{aligned}
& \lambda_{i}\left(\widehat{\mathbf{H}} \widehat{\mathbf{H}}^{H}\right)=\lambda_{i}\left(\boldsymbol{\Theta}^{H}\right), \quad i=1,2, \ldots, M_{\mathrm{R}}, \quad M_{\mathrm{R}} \leq M_{\mathrm{T}} \\
& \lambda_{i}\left(\widehat{\mathbf{H}}^{H} \widehat{\mathbf{H}}\right)=\lambda_{i}\left(\boldsymbol{\Theta}^{H} \boldsymbol{\Theta}\right), \quad i=1,2, \ldots, M_{\mathrm{T}}, \quad M_{\mathrm{R}}>M_{\mathrm{T}} .
\end{aligned}
$$

Proof: The proof for both cases is trivially obtained using Lemma 2 and noting that the assumptions of the Theorem imply $\operatorname{diag}(\mathbf{g})(\operatorname{diag}(\mathbf{g}))^{H}=\mathbf{I}_{M_{\mathrm{R}}}$ and $\operatorname{diag}(\mathbf{h})(\operatorname{diag}(\mathbf{h}))^{H}=\mathbf{I}_{M_{\mathrm{T}}}$. For $M_{\mathrm{R}} \leq M_{\mathrm{T}}$, simply note that

$$
\begin{aligned}
\lambda_{i}\left(\widehat{\mathbf{H}} \widehat{\mathbf{H}}^{H}\right) & =\lambda_{i}\left(\operatorname{diag}(\mathbf{g}) \boldsymbol{\Theta} \operatorname{diag}(\mathbf{h})(\operatorname{diag}(\mathbf{h}))^{H} \boldsymbol{\Theta}^{H}(\operatorname{diag}(\mathbf{g}))^{H}\right) \\
& =\lambda_{i}\left((\operatorname{diag}(\mathbf{g}))^{H} \operatorname{diag}(\mathbf{g}) \boldsymbol{\Theta} \boldsymbol{\Theta}^{H}\right) \\
& =\lambda_{i}\left(\boldsymbol{\Theta} \boldsymbol{\Theta}^{H}\right), \quad i=1,2, \ldots, M_{\mathrm{R}} .
\end{aligned}
$$

The case $M_{\mathrm{R}}>M_{\mathrm{T}}$ follows exactly the same line of reasoning. 
Since the high-SNR MI of $\widehat{\mathbf{H}}$ (for $M_{\mathrm{R}} \leq M_{\mathrm{T}}$ ) is given by $\hat{I} \approx \log \operatorname{det}\left(\left(\rho / M_{\mathrm{T}}\right) \widehat{\mathbf{H}} \widehat{\mathbf{H}}^{H}\right)$, Theorems 2 and 3 immediately yield expressions 9 for the high-SNR MI of $\widehat{\mathbf{H}}$. However, the pdf of the quantity $\log \operatorname{det}\left(\Theta \Theta^{H}\right)$ is, in general, difficult to obtain. Insightful analytic results are, however, possible by invoking the assumptions of a separable timing matrix (as discussed in Section IV-A3 and of small phase noise, i.e., $\exp ^{\circ}(j \Phi) \approx 1+j \boldsymbol{\Phi}$. As demonstrated previously, a separable timing matrix is obtained by choosing a regular sounding pattern as in (26) and the small phase noise approximation is very well satisfied in practice as the worst-case value of $7^{\circ}$ rms phase noise amounts to $\sigma_{\varphi}^{2} \approx 0.0149$. The assumption of a separable timing matrix implies that frequency offset has no impact on MI (see Section IV-A3). Therefore, as a consequence of the two simplifying assumptions, it suffices to analyze the quantity $\operatorname{det}\left(\widetilde{\boldsymbol{\Theta}} \widetilde{\Theta}^{H}\right)$ with $\widetilde{\boldsymbol{\Theta}}=\mathbf{1}+j \boldsymbol{\Phi}$ instead of $\operatorname{det}\left(\Theta \Theta^{H}\right)$. Interestingly, $\operatorname{det}\left(\widetilde{\boldsymbol{\Theta}} \widetilde{\Theta}^{H}\right)$ can be characterized in terms of chi-square RVs and a beta-distributed RV, which provides the basis for tight bounds on $\widehat{C}=\mathbb{E}\{\hat{I}\}$ and for accurate approximations of $\operatorname{Var}\{\hat{I}\}$. Before stating the corresponding results using the exact expression for $\operatorname{det}\left(\widetilde{\boldsymbol{\Theta}} \widetilde{\Theta}{ }^{H}\right)$, we shall, however, provide an approximation for $\operatorname{det}\left(\widetilde{\boldsymbol{\Theta}} \widetilde{\Theta}^{H}\right)$ (in the sense of distributional equivalence), which turns out to be particularly useful to derive a simple analytic lower bound on $\widehat{C}$ (see Theorem 8 ). This approximation is based on the following result.

Theorem 5: For a separable timing matrix $\mathbf{T}$ and under the small phase noise approximation $\sigma_{\varphi}^{2} \ll 1$ so that $\widetilde{\boldsymbol{\Theta}}=\mathbf{1}+j \boldsymbol{\Phi}$, assuming fully uncorrelated phase noise, i.e., $\operatorname{vec}(\boldsymbol{\Phi}) \stackrel{\mathrm{d}}{=}$ $\mathcal{N}\left(\mathbf{0}, \sigma_{\varphi}^{2} \mathbf{I}_{M_{\mathrm{T}} M_{\mathrm{R}}}\right)$, we have

$$
\begin{array}{ll}
\operatorname{det}\left(\widetilde{\boldsymbol{\Theta}} \widetilde{\Theta}^{H}\right) \stackrel{\mathrm{d}}{=}\left(\chi_{M_{\mathrm{T}}, \sigma_{\varphi}^{2}}^{2}+M_{\mathrm{T}} M_{\mathrm{R}}\right) \prod_{i=2}^{M_{\mathrm{R}}}\left(\chi_{M_{\mathrm{T}}-i, \sigma_{\varphi}^{2}}^{2}+Z\left(\eta^{(i)}\right)\right), \quad M_{\mathrm{R}} \leq M_{\mathrm{T}} \\
\operatorname{det}\left(\widetilde{\boldsymbol{\Theta}}^{H} \widetilde{\boldsymbol{\Theta}}\right) \stackrel{\mathrm{d}}{=}\left(\chi_{M_{\mathrm{R}}, \sigma_{\varphi}^{2}}^{2}+M_{\mathrm{T}} M_{\mathrm{R}}\right) \prod_{i=2}^{M_{\mathrm{T}}}\left(\chi_{M_{\mathrm{R}}-i, \sigma_{\varphi}^{2}}^{2}+Z\left(\eta^{(i)}\right)\right), \quad M_{\mathrm{R}}>M_{\mathrm{T}}
\end{array}
$$

\footnotetext{
${ }^{9}$ More specifically an approximation in the case of Theorem 2 due to the presence of the quantities $\varepsilon_{i}$ and $\nu_{i}$.
} 
where the $\chi_{n, \sigma^{2}}^{2}$ are statistically independent 10 and $Z\left(\eta^{(i)}\right)=\sigma_{\varphi}^{2}\left(\eta^{(i)} X_{1}^{(i)}+\left(1-\eta^{(i)}\right) X_{2}^{(i)}\right)$ with $X_{1}^{(i)}, X_{2}^{(i)}$ i.i.d. as $\chi_{1,1}^{2}$ and the $\eta^{(i)}$ being RVs with pdf supported in the interval [0,1] $\forall i$.

Proof: We provide the proof for $M_{\mathrm{R}} \leq M_{\mathrm{T}}$ only. The case $M_{\mathrm{R}}>M_{\mathrm{T}}$ follows exactly the same line of reasoning. Let us start by noting that the singular value decomposition of $\mathbf{1}_{M_{\mathrm{R}}, M_{\mathrm{T}}}$ is given by $\mathbf{1}_{M_{\mathrm{R}}, M_{\mathrm{T}}}=\mathbf{V} \boldsymbol{\Sigma} \mathbf{W}^{T}$, where $\mathbf{V}$ is of dimension $M_{\mathrm{R}} \times M_{\mathrm{R}}, \mathbf{W}$ is $M_{\mathrm{T}} \times M_{\mathrm{T}}$, and the $M_{\mathrm{R}} \times M_{\mathrm{T}}$ matrix $\Sigma$ is given by

$$
[\Sigma]_{m, n}= \begin{cases}\sqrt{M_{\mathrm{T}} M_{\mathrm{R}}}, & m=n=1 \\ 0, & \text { else. }\end{cases}
$$

Defining the $M_{\mathrm{R}} \times M_{\mathrm{T}}$ matrix $\mathbf{S}=-j \boldsymbol{\Sigma}+\widetilde{\boldsymbol{\Phi}}$ with $\widetilde{\boldsymbol{\Phi}}=\mathbf{V}^{T} \boldsymbol{\Phi} \mathbf{W}$ (and hence $\widetilde{\boldsymbol{\Phi}} \stackrel{\mathrm{d}}{=} \boldsymbol{\Phi}$ ), it follows that $\operatorname{det}\left(\widetilde{\boldsymbol{\Theta}} \widetilde{\boldsymbol{\Theta}}^{H}\right)=\operatorname{det}\left(\mathbf{S S}^{H}\right)$. With $\mathbf{S}=\left[\begin{array}{llll}\mathbf{s}_{1} & \mathbf{s}_{2} & \cdots & \mathbf{s}_{M_{\mathrm{R}}}\end{array}\right]^{T}$ being a square $\left(M_{\mathrm{R}}=M_{\mathrm{T}}\right)$ or a wide matrix $\left(M_{\mathrm{R}}<M_{\mathrm{T}}\right)$, a basic result in geometry (e.g., [47, Th. 7.5.1], [48, Sec. 3.2.2], which can be shown to hold in the complex case upon replacing transposition by conjugate transposition) yields

$$
\sqrt{\operatorname{det}\left(\mathbf{S S}^{H}\right)}=\operatorname{vol}\left(P_{\mathbf{S}}\right)=\left\|\mathbf{s}_{1}^{\perp}\right\|\left\|\mathbf{s}_{2}^{\perp}\right\| \cdots\left\|\mathbf{s}_{M_{\mathrm{R}}}^{\perp}\right\|
$$

where $\operatorname{vol}\left(P_{\mathbf{S}}\right)$ stands for the volume or $M_{\mathrm{R}}$-content of the parallelotope spanned by the $M_{\mathrm{R}}$ row vectors of $\mathbf{S}, \mathbf{s}_{1}^{\perp}=\mathbf{s}_{1}$, and $\mathbf{s}_{i}^{\perp}(i>1)$ denotes the component of $\mathbf{s}_{i}$ orthogonal to the span of the vectors $\mathbf{s}_{1}^{\perp}, \mathbf{s}_{2}^{\perp}, \ldots, \mathbf{s}_{i-1}^{\perp}$. The orthogonal vectors $\mathbf{s}_{i}^{\perp}\left(i=2,3, \ldots, M_{\mathrm{R}}\right)$ are obtained using Gram-Schmidt orthogonalization and are given by

$$
\mathbf{s}_{i}^{\perp}=\left(\mathbf{I}_{M_{\mathrm{T}}}-\sum_{n=1}^{i-1} \frac{\mathbf{s}_{n}^{\perp} \mathbf{s}_{n}^{\perp H}}{\left\|\mathbf{s}_{n}^{\perp}\right\|^{2}}\right) \mathbf{s}_{i}=\mathbf{A}_{i} \mathbf{s}_{i} .
$$

It is well known that applying the decomposition (52) to an i.i.d. complex Gaussian random matrix $\mathbf{S}$ with $\mathcal{C N}(0,1)$ elements results in independent chi-square distributed factors $\left\|\mathbf{s}_{i}^{\perp}\right\|^{2}$

\footnotetext{
${ }^{10}$ Note that the product over $i$ on the RHS of (50) is equal to 1 if $M_{\mathrm{R}}=1$ (in the case $M_{\mathrm{R}} \leq M_{\mathrm{T}}$ ) and $M_{\mathrm{T}}=1$ (in the case $M_{\mathrm{R}}>M_{\mathrm{T}}$ ).
} 
$\left(i=1,2, \ldots, M_{\mathrm{R}}\right)[49$, Th. $3.4 \mathrm{ff}$.$] . The problem at hand differs, however, from the i.i.d. complex$ Gaussian case in two aspects, namely the fact that the elements in $\Phi$ and hence $\widetilde{\Phi}$ are real-valued Gaussian and the presence of the deterministic component $-j \Sigma$.

It follows trivially from the definition of $\mathbf{S}$ that $\left\|\mathbf{s}_{1}^{\perp}\right\|^{2} \stackrel{\mathrm{d}}{=} \chi_{M_{\mathrm{T}}, \sigma_{\varphi}^{2}}^{2}+M_{\mathrm{T}} M_{\mathrm{R}}$. From (53) we can see that, conditioned on $\mathbf{s}_{1}^{\perp}, \mathbf{s}_{2}^{\perp}, \ldots, \mathbf{s}_{i-1}^{\perp}$, the vectors $\mathbf{s}_{i}^{\perp}\left(i=2,3, \ldots, M_{\mathrm{R}}\right)$ are JG and hence the $\left\|\mathbf{s}_{i}^{\perp}\right\|^{2}\left(i=2,3, \ldots, M_{\mathrm{R}}\right)$ are chi-square distributed. Using the fact that $\mathbf{s}_{i} \in \mathbb{R}^{M_{\mathrm{T}}}$ $\left(i=2,3, \ldots, M_{\mathrm{R}}\right)$ and $\mathbf{A}_{i}^{H} \mathbf{A}_{i}=\mathbf{A}_{i}$, it follows immediately that

$$
\left\|\mathbf{s}_{i}^{\perp}\right\|^{2}=\mathbf{s}_{i}^{T} \mathbf{A}_{i} \mathbf{s}_{i}, \quad i=2,3, \ldots, M_{\mathrm{R}}
$$

Next, noting that

$$
\begin{aligned}
\left\|\mathbf{s}_{i}^{\perp}\right\|^{2} & =\mathbf{s}_{i}^{T}\left(\operatorname{Re}\left(\mathbf{A}_{i}\right)+j \operatorname{Im}\left(\mathbf{A}_{i}\right)\right) \mathbf{s}_{i} \\
& =\mathbf{s}_{i}^{T} \operatorname{Re}\left(\mathbf{A}_{i}\right) \mathbf{s}_{i}+j \mathbf{s}_{i}^{T} \operatorname{Im}\left(\mathbf{A}_{i}\right) \mathbf{s}_{i}, \quad i=2,3, \ldots, M_{\mathrm{R}}
\end{aligned}
$$

has to be real-valued for all $\mathbf{s}_{i}$, it follows that

$$
\left\|\mathbf{s}_{i}^{\perp}\right\|^{2}=\mathbf{s}_{i}^{T} \operatorname{Re}\left(\mathbf{A}_{i}\right) \mathbf{s}_{i}=\mathbf{s}_{i}^{T}\left(\mathbf{I}_{M_{\mathrm{T}}}-\sum_{n=1}^{i-1} \frac{\operatorname{Re}\left(\mathbf{s}_{n}^{\perp} \mathbf{s}_{n}^{\perp H}\right)}{\left\|\mathbf{s}_{n}^{\perp}\right\|^{2}}\right) \mathbf{s}_{i} .
$$

Based on (54), we can now invoke Lemma 5 in the Appendix to conclude that the eigenvalues of $\operatorname{Re}\left(\mathbf{A}_{i}\right)$ are given by

$$
\left\{\sigma_{k}^{(i)}\right\}=\{\underbrace{1, \ldots, 1}_{M_{\mathrm{T}}-i}, \underbrace{0, \ldots, 0}_{i-2}, \eta^{(i)}, 1-\eta^{(i)}\}, \quad k=1,2, \ldots, M_{\mathrm{T}}
$$

where $\eta^{(i)}=\eta^{(i)}\left(\mathbf{s}_{1}^{\perp}, \mathbf{s}_{2}^{\perp}, \ldots, \mathbf{s}_{i-1}^{\perp}\right)$ is a RV with pdf supported in the interval $[0,1]$. Consequently, using [41, Eq. (4.1.1)], we obtain

$$
\mathbf{s}_{i}^{T} \operatorname{Re}\left(\mathbf{A}_{i}\right) \mathbf{s}_{i} \stackrel{\mathrm{d}}{=} \sigma_{\varphi}^{2} \sum_{k=1}^{M_{\mathrm{T}}} \sigma_{k}^{(i)} X_{i}=\chi_{M_{\mathrm{T}}-i, \sigma_{\varphi}^{2}}^{2}+\underbrace{\sigma_{\varphi}^{2}\left(\eta^{(i)} X_{M_{\mathrm{T}}-1}^{(i)}+\left(1-\eta^{(i)}\right) X_{M_{\mathrm{T}}}^{(i)}\right)}_{\stackrel{\mathrm{d}}{=} Z\left(\eta^{(i)}\right)}
$$


where the $X_{i} \stackrel{\mathrm{d}}{=} \chi_{1,1}^{2}$ are independent.

We shall next show that for $\sigma_{\varphi}^{2} \ll 1, Z\left(\eta^{(i)}\right) \stackrel{\mathrm{d}}{\approx} \chi_{1, \sigma_{\varphi}^{2}}^{2}$, which then implies that

$$
\left\|\mathbf{s}_{i}^{\perp}\right\|^{2} \stackrel{\mathrm{d}}{\approx} \chi_{M_{\mathrm{T}}-i, \sigma_{\varphi}^{2}}^{2}+\chi_{1, \sigma_{\varphi}^{2}}^{2}=\chi_{M_{\mathrm{T}}-i+1, \sigma_{\varphi}^{2}}^{2}
$$

thereby allowing an approximation of (50) a

$$
\begin{array}{ll}
\operatorname{det}\left(\widetilde{\boldsymbol{\Theta}}^{H}\right) \stackrel{d}{\approx}\left(\chi_{M_{\mathrm{T}}, \sigma_{\varphi}^{2}}^{2}+M_{\mathrm{T}} M_{\mathrm{R}}\right) \prod_{i=1}^{M_{\mathrm{R}}-1} \chi_{M_{\mathrm{T}}-i, \sigma_{\varphi}^{2}}^{2}, & M_{\mathrm{R}} \leq M_{\mathrm{T}} \\
\operatorname{det}\left(\widetilde{\boldsymbol{\Theta}}^{H} \widetilde{\boldsymbol{\Theta}}\right) \stackrel{d}{\approx}\left(\chi_{M_{\mathrm{R}}, \sigma_{\varphi}^{2}}^{2}+M_{\mathrm{T}} M_{\mathrm{R}}\right) \prod_{i=1}^{M_{\mathrm{T}}-1} \chi_{M_{\mathrm{R}}-i, \sigma_{\varphi}^{2}}^{2}, & M_{\mathrm{R}}>M_{\mathrm{T}} .
\end{array}
$$

In order to see that $Z\left(\eta^{(i)}\right) \stackrel{\mathrm{d}}{\approx} \chi_{1, \sigma_{\varphi}^{2}}^{2}$, we start by noting that the pdf of $Z(\eta)$ conditional on $\eta$ is given by [51, Eq. (5.7)]

$$
\mathrm{p}_{Z \mid \eta}(x)=\frac{1}{2 \sigma_{\varphi}^{2} \sqrt{\eta(1-\eta)}} e^{-\frac{x}{4 \sigma_{\varphi}^{2} \eta(1-\eta)}} I_{0}\left(\frac{1-2 \eta}{4 \sigma_{\varphi}^{2} \eta(1-\eta)} x\right)
$$

where $I_{0}(z)$ is the modified Bessel function of the first kind [15, Sec. 9.6]. For $\sigma_{\varphi}^{2}$ small, we can invoke the large- $|z|$ expansion of $I_{0}(z)$ [15, Eq. 9.7.1] according to

$$
I_{0}(z)=\frac{1}{\sqrt{2 \pi z}} e^{z}\left(1+\frac{1}{8 z}+\frac{3^{2}}{2 !(8 z)^{2}}+\frac{3^{2} 5^{2}}{3 !(8 z)^{3}}+\cdots\right) \approx \frac{1}{\sqrt{2 \pi z}} e^{z}
$$

which, when used in (57), upon renormalizing so that $\int_{x=0}^{\infty} \mathrm{p}_{Z \mid \eta}(x) \mathrm{d} x=1$, yields

$$
\mathrm{p}_{Z \mid \eta}(x) \approx \frac{1}{\sqrt{2 \pi \sigma_{\varphi}^{2}(1-\eta) x}} e^{-\frac{x}{2 \sigma_{\varphi}^{2}(1-\eta)}}=\mathrm{p}_{\chi_{1, \sigma_{\varphi}^{2}(1-\eta)}^{2}}(x) .
$$

This means that $Z \mid \eta \stackrel{\mathrm{d}}{=} \chi_{1, \sigma_{\varphi}^{2}(1-\eta)}^{2}$ for $0<\eta<1$ if $\sigma_{\varphi}^{2}$ is small. We shall next see that $\eta^{(i)}, \forall i$, is small, in general, which then directly results in the (unconditional) pdf of $Z\left(\eta^{(i)}\right)$ satisfying $Z\left(\eta^{(i)}\right) \stackrel{\mathrm{d}}{\approx} \chi_{1, \sigma_{\varphi}^{2}}^{2}, \forall i$. Recall that $\left\{\eta^{(i)}, 1-\eta^{(i)}\right\}$ are the nonzero, nonunity eigenvalues of $\operatorname{Re}\left(\mathbf{A}_{i}\right)$

\footnotetext{
${ }^{11}$ We would like to use this chance to point out that the distributional equivalence in [50, Prop. 4] should be an approximate equivalence (as in (56). Furthermore, $\mathcal{C N}\left(\mathbf{0}, \sigma_{\Phi}^{2} \mathbf{I}_{M_{\mathrm{T}} M_{\mathrm{R}}}\right)$ in [50, Prop. 4 and Prop. 5] should be replaced by $\mathcal{N}\left(\mathbf{0}, \sigma_{\Phi}^{2} \mathbf{I}_{M_{\mathrm{T}} M_{\mathrm{R}}}\right)$.
} 
in (54). The first pair of such eigenvalues is obtained for $i=2$. Due to the symmetry of the eigenvalues, we may investigate $\mathbf{I}-\operatorname{Re}\left(\mathbf{A}_{2}\right)$ instead of $\operatorname{Re}\left(\mathbf{A}_{2}\right)$, which, using $\mathbf{s}_{1}^{\perp}=\mathbf{s}_{1}$, can be written as

$$
\begin{aligned}
\mathbf{I}-\operatorname{Re}\left(\mathbf{A}_{2}\right) & =\frac{\operatorname{Re}\left(\mathbf{s}_{1} \mathbf{s}_{1}^{H}\right)}{\left\|\mathbf{s}_{1}\right\|^{2}}=\frac{\operatorname{Re}\left(\mathbf{s}_{1}\right)\left(\operatorname{Re}\left(\mathbf{s}_{1}\right)\right)^{T}}{\left\|\mathbf{s}_{1}\right\|^{2}}+\frac{\operatorname{Im}\left(\mathbf{s}_{1}\right)\left(\operatorname{Im}\left(\mathbf{s}_{1}\right)\right)^{T}}{\left\|\mathbf{s}_{1}\right\|^{2}} \\
& =\frac{\boldsymbol{\varphi} \boldsymbol{\varphi}^{T}}{\|\boldsymbol{\varphi}\|^{2}+\|\boldsymbol{\sigma}\|^{2}}+\frac{\boldsymbol{\sigma} \boldsymbol{\sigma}^{T}}{\|\boldsymbol{\varphi}\|^{2}+\|\boldsymbol{\sigma}\|^{2}}
\end{aligned}
$$

where $\boldsymbol{\varphi}=\operatorname{Re}\left(\mathbf{s}_{1}\right)$ and $\boldsymbol{\sigma}=-\operatorname{Im}\left(\mathbf{s}_{1}\right)=\left[\begin{array}{lllll}\sqrt{M_{\mathrm{T}} M_{\mathrm{R}}} & 0 & 0 & \cdots & 0\end{array}\right]^{T}$. In the following, we denote $\varphi_{i}=[\boldsymbol{\varphi}]_{i}$. The nonzero eigenvalues of $\mathbf{I}-\operatorname{Re}\left(\mathbf{A}_{2}\right)$ are equal to the eigenvalues of

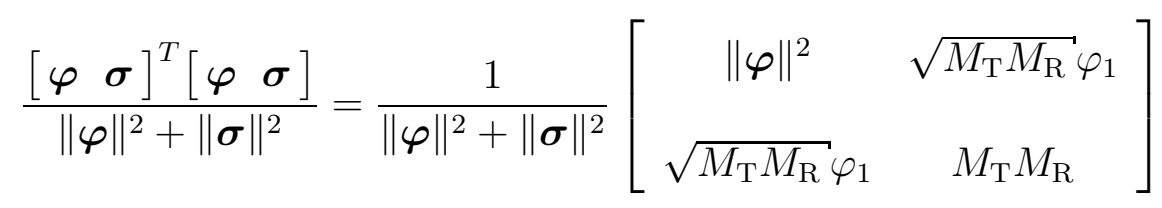

given by

$$
\left\{\eta^{(2)}, 1-\eta^{(2)}\right\}=\frac{1}{2} \pm \frac{1}{2} \frac{\sqrt{4 M_{\mathrm{T}} M_{\mathrm{R}} \varphi_{1}^{2}+\left(M_{\mathrm{T}} M_{\mathrm{R}}-\|\boldsymbol{\varphi}\|^{2}\right)^{2}}}{M_{\mathrm{T}} M_{\mathrm{R}}+\|\boldsymbol{\varphi}\|^{2}}
$$

For $M_{\mathrm{T}}$ sufficiently large, with $\|\varphi\|^{2}=\varphi_{1}^{2}+\varphi_{\mathrm{s}}^{2}$, where $\varphi_{\mathrm{s}}^{2}=\sum_{i=2}^{M_{\mathrm{T}}} \varphi_{i}^{2}$, we can replace (58) by

$$
\left\{\eta^{(2)}, 1-\eta^{(2)}\right\} \approx \frac{1}{2} \pm \frac{1}{2} \frac{M_{\mathrm{T}} M_{\mathrm{R}}-\varphi_{\mathrm{s}}^{2}}{M_{\mathrm{T}} M_{\mathrm{R}}+\varphi_{\mathrm{s}}^{2}}
$$

Next, since $\varphi_{\mathrm{S}}^{2}$ is small compared to $M_{\mathrm{T}} M_{\mathrm{R}}$, we obtain the first-order Taylor series expansion

$$
\left\{\eta^{(2)}, 1-\eta^{(2)}\right\} \approx \frac{1}{2} \pm \frac{1}{2}\left(1-2 \frac{\varphi_{\mathrm{s}}^{2}}{M_{\mathrm{T}} M_{\mathrm{R}}}\right) \stackrel{\mathrm{d}}{=}\left\{\chi_{M_{\mathrm{T}}-1, \sigma_{\varphi}^{2} /\left(M_{\mathrm{T}} M_{\mathrm{R}}\right)}^{2}, 1-\chi_{M_{\mathrm{T}}-1, \sigma_{\varphi}^{2} /\left(M_{\mathrm{T}} M_{\mathrm{R}}\right)}^{2}\right\}
$$

Hence, we have

$$
\mathbb{E}\left\{\eta^{(2)}\right\}=\sigma_{\varphi}^{2} \frac{M_{\mathrm{T}}-1}{M_{\mathrm{T}} M_{\mathrm{R}}} \quad \operatorname{Var}\left\{\eta^{(2)}\right\}=2 \sigma_{\varphi}^{4} \frac{M_{\mathrm{T}}-1}{M_{\mathrm{T}}^{2} M_{\mathrm{R}}^{2}}
$$

which shows that, for sufficiently large $M_{\mathrm{T}}, M_{\mathrm{R}}, \eta^{(2)}$ is indeed small. For $M_{\mathrm{R}} \times 1$ and $1 \times M_{\mathrm{T}}$ 
systems, i.e., for SIMO and multiple-input single-output (MISO) systems, respectively, we can therefore immediately conclude that the approximation (56) is very accurate. In the case of general $M_{\mathrm{T}}$ and $M_{\mathrm{R}}$, it seems difficult to prove that $\eta^{(i)} \approx 0$ for $i \geq 3$. We do, however, have strong numerical evidence that this is, indeed, the case.

Recalling that $\sigma_{\varphi}^{2} \approx 0.0149$ for the worst-case phase noise value of $7^{\circ} \mathrm{rms}$, we can conclude that the assumption $\sigma_{\varphi}^{2} \ll 1$ made in Theorem 5 and in (56) is very well satisfied in practice. Fig. 6 shows the cdf of $\log \operatorname{det}\left(\widetilde{\Theta} \widetilde{\Theta}^{H}\right)$ corresponding to the approximation (56) along with the exact $\mathrm{cdf}^{12}$ (in both cases obtained through Monte Carlo methods). We observe that the approximation is excellent in general and, indeed, becomes better for smaller $\sigma_{\varphi}^{2}$ and/or for less symmetric (in terms of the number of transmit and receive antennas) configurations. We finally note that comparing (56) to [52, Eq. (3)] suggests that for fully uncorrelated phase noise with $\sigma_{\varphi}^{2} \ll 1$, the effective MIMO channel behaves like a physical MIMO channel consisting of a rank-1 Ricean component plus an i.i.d. Rayleigh fading component with the difference that in our case the chi-square RVs have half the order of those in [52] (reflecting the fact that here we are dealing with real-valued Gaussian RVs).

We proceed by stating the result on the exact $\operatorname{distribution} \operatorname{det}\left(\widetilde{\boldsymbol{\Theta}} \widetilde{\Theta}^{H}\right)$.

Theorem 6: For a separable timing matrix $\mathbf{T}$ and under the small phase noise approximation $\sigma_{\varphi}^{2} \ll 1$ so that $\widetilde{\boldsymbol{\Theta}}=\mathbf{1}+j \boldsymbol{\Phi}$, assuming fully uncorrelated phase noise, i.e., $\operatorname{vec}(\boldsymbol{\Phi}) \stackrel{\mathrm{d}}{=}$ $\mathcal{N}\left(\mathbf{0}, \sigma_{\varphi}^{2} \mathbf{I}_{M_{\mathrm{T}} M_{\mathrm{R}}}\right)$, we have

$$
\begin{aligned}
& \operatorname{det}\left(\widetilde{\boldsymbol{\Theta}} \widetilde{\boldsymbol{\Theta}}^{H}\right) \stackrel{\mathrm{d}}{=}\left(\chi_{M_{\mathrm{d}}+1, \sigma_{\varphi}^{2}}^{2}+M_{\mathrm{T}} M_{\mathrm{R}} \beta\left(\frac{M_{\mathrm{d}}+1}{2}, \frac{M_{\mathrm{R}}-1}{2}\right)\right) \prod_{i=1}^{M_{\mathrm{R}}-1} \chi_{M_{\mathrm{T}}-i+1, \sigma_{\varphi}^{2}}^{2}, \quad M_{\mathrm{R}} \leq M_{\mathrm{T}} \\
& \operatorname{det}\left(\widetilde{\boldsymbol{\Theta}} \widetilde{\boldsymbol{\Theta}}^{H} \stackrel{\mathrm{d}}{=}\left(\chi_{M_{\mathrm{d}}+1, \sigma_{\varphi}^{2}}^{2}+M_{\mathrm{T}} M_{\mathrm{R}} \beta\left(\frac{M_{\mathrm{d}}+1}{2}, \frac{M_{\mathrm{T}}-1}{2}\right)\right) \prod_{i=1}^{M_{\mathrm{T}}-1} \chi_{M_{\mathrm{R}}-i+1, \sigma_{\varphi}^{2}}^{2}, \quad M_{\mathrm{R}}>M_{\mathrm{T}}\right.
\end{aligned}
$$

\footnotetext{
${ }^{12}$ Note that "exact cdf" means exact under the linear phase noise approximation.
} 
where $M_{\mathrm{d}}=\left|M_{\mathrm{T}}-M_{\mathrm{R}}\right|$, and the $\chi_{n, \sigma^{2}}^{2}$ are statistically independent.

Proof: Again, we provide the proof for $M_{\mathrm{R}} \leq M_{\mathrm{T}}$ only. The case $M_{\mathrm{R}}>M_{\mathrm{T}}$ follows exactly the same line of reasoning. We start by noting that the matrix $\mathbf{S}$ defined in the proof of Theorem 5 is unitarily equivalent to the matrix $\mathbf{S}^{\prime}=-j \boldsymbol{\Sigma}^{\prime}+\widetilde{\boldsymbol{\Phi}}^{\prime}$, where the $M_{\mathrm{R}} \times M_{\mathrm{T}}$ matrix $\Sigma^{\prime}$ is given by

$$
\left[\boldsymbol{\Sigma}^{\prime}\right]_{m, n}= \begin{cases}\sqrt{M_{\mathrm{T}} M_{\mathrm{R}}}, & m=n=M_{\mathrm{R}} \\ 0, & \text { else }\end{cases}
$$

and $\widetilde{\boldsymbol{\Phi}}^{\prime} \stackrel{\mathrm{d}}{=} \widetilde{\boldsymbol{\Phi}} \stackrel{\mathrm{d}}{=} \boldsymbol{\Phi}$. In what follows, we shall work with $\mathrm{S}^{\prime}$ and, by slight abuse of notation, denote it as $\mathbf{S}$. The pdf of $\left\|\mathbf{s}_{1}^{\perp}\right\|^{2}$ follows trivially from the definition of $\mathbf{S}$ and is given by $\chi_{M_{\mathrm{T}}, \sigma_{\varphi}^{2}}^{2}$. Applying Gram-Schmidt orthogonalization, due to the nonzero entry in $\Sigma^{\prime}$ being at position $m=n=M_{\mathrm{R}}$, we can conclude that for $i=2,3, \ldots, M_{\mathrm{R}}-1$, the matrix $\operatorname{Re}\left(\mathbf{A}_{i}\right)$ has only two distinct eigenvalues, namely 0 with multiplicity $i-1$ and 1 with multiplicity $M_{\mathrm{T}}-i+1$. Consequently, (54) implies that $\left\|\mathbf{s}_{i}^{\perp}\right\|^{2}\left(i=1,2, \ldots, M_{\mathrm{R}}-1\right)$ conditioned on $\mathbf{s}_{1}^{\perp}, \mathbf{s}_{2}^{\perp}, \ldots, \mathbf{s}_{i-1}^{\perp}$ is distributed as $\chi_{M_{\mathrm{T}}-i+1, \sigma_{\varphi}^{2}}^{2}$. Since the eigenvalues of $\operatorname{Re}\left(\mathbf{A}_{i}\right)$ do not depend on $\mathbf{s}_{1}^{\perp}, \mathbf{s}_{2}^{\perp}, \ldots, \mathbf{s}_{i-1}^{\perp}$ and the statistics of $\left\|\mathbf{s}_{i}^{\perp}\right\|^{2}$ depend on $\mathbf{A}_{i}$ only through the eigenvalues of $\mathbf{A}_{i}$, we can conclude that the unconditional distribution of $\left\|\mathbf{s}_{i}^{\perp}\right\|^{2}$ satisfies $\left\|\mathbf{s}_{i}^{\perp}\right\|^{2} \stackrel{\mathrm{d}}{=} \chi_{M_{\mathrm{T}}-i+1, \sigma_{\varphi}^{2}}^{2}\left(i=1,2, \ldots, M_{\mathrm{R}}-1\right)$. For $i=M_{\mathrm{R}}$, noting that $\mathbf{A}_{M_{\mathrm{R}}}$ is a real-valued matrix, we have

$$
\left\|\mathbf{s}_{M_{\mathrm{R}}}^{\perp}\right\|^{2}=\left\|\mathbf{A}_{M_{\mathrm{R}}} \operatorname{Re}\left(\mathbf{s}_{M_{\mathrm{R}}}\right)\right\|^{2}+\left\|\mathbf{A}_{M_{\mathrm{R}}} \operatorname{Im}\left(\mathbf{s}_{M_{\mathrm{R}}}\right)\right\|^{2} .
$$

The distribution of the first term on the RHS of (60) can be shown, using the same line of reasoning as for $i=1,2, \ldots, M_{\mathrm{R}}-1$, to satisfy $\left\|\mathbf{A}_{M_{\mathrm{R}}} \operatorname{Re}\left(\mathbf{s}_{M_{\mathrm{R}}}\right)\right\|^{2} \stackrel{\mathrm{d}}{=} \chi_{M_{\mathrm{T}}-M_{\mathrm{R}}+1, \sigma_{\varphi}^{2}}^{2}$. The second term on the RHS of (60) can be expanded as

$$
\left\|\mathbf{A}_{M_{\mathrm{R}}} \operatorname{Im}\left(\mathbf{s}_{M_{\mathrm{R}}}\right)\right\|^{2}=\left(\operatorname{Im}\left(\mathbf{s}_{M_{\mathrm{R}}}\right)\right)^{T} \mathbf{A}_{M_{\mathrm{R}}}^{T} \mathbf{A}_{M_{\mathrm{R}}} \operatorname{Im}\left(\mathbf{s}_{M_{\mathrm{R}}}\right)
$$




$$
=\left(\operatorname{Im}\left(\mathbf{s}_{M_{\mathrm{R}}}\right)\right)^{T} \mathbf{A}_{M_{\mathrm{R}}} \operatorname{Im}\left(\mathbf{s}_{M_{\mathrm{R}}}\right)
$$

where we made use of the fact that $\mathbf{A}_{M_{\mathrm{R}}}$ is real-valued and hence $\mathbf{A}_{M_{\mathrm{R}}}^{H} \mathbf{A}_{M_{\mathrm{R}}}=\mathbf{A}_{M_{\mathrm{R}}}$ reduces to $\mathbf{A}_{M_{\mathrm{R}}}^{T} \mathbf{A}_{M_{\mathrm{R}}}=\mathbf{A}_{M_{\mathrm{R}}}$. Next, we note that

$$
\begin{aligned}
\mathbf{A}_{M_{\mathrm{R}}} & =\mathbf{I}_{M_{\mathrm{T}}}-\mathbf{G G}^{T} \\
& =\left[\begin{array}{ll}
\mathbf{G} & \mathbf{K}
\end{array}\right]\left[\begin{array}{cc}
\mathbf{0} & \mathbf{0} \\
\mathbf{0} & \mathbf{I}_{M_{\mathrm{T}}-M_{\mathrm{R}}+1}
\end{array}\right]\left[\begin{array}{ll}
\mathbf{G} & \mathbf{K}
\end{array}\right]^{T} \\
& =\sum_{n=M_{\mathrm{R}}}^{M_{\mathrm{T}}} \mathbf{u}_{n} \mathbf{u}_{n}^{T}
\end{aligned}
$$

with

$$
\mathbf{G}=\left[\begin{array}{llll}
\frac{\mathbf{s}_{1}^{\perp}}{\left\|\mathbf{s}_{1}^{\perp}\right\|} & \frac{\mathbf{s}_{2}^{\perp}}{\left\|\mathbf{s}_{2}^{\perp}\right\|} & \cdots & \frac{\mathbf{s}_{M_{\mathrm{R}}-1}^{\perp}}{\left\|\mathbf{s}_{M_{\mathrm{R}}-1}^{\perp}\right\|}
\end{array}\right] \quad \text { and } \quad \mathbf{K}=\left[\begin{array}{llll}
\mathbf{u}_{M_{\mathrm{R}}} & \mathbf{u}_{M_{\mathrm{R}}+1} & \cdots & \mathbf{u}_{M_{\mathrm{T}}}
\end{array}\right]
$$

and the vectors $\mathbf{u}_{n}\left(n=M_{\mathrm{R}}, M_{\mathrm{R}}+1, \ldots, M_{\mathrm{T}}\right)$ have to be chosen such that the matrix $\mathbf{U}=$ $\left[\begin{array}{ll}\mathbf{G} & \mathbf{K}\end{array}\right]$ satisfies $\mathbf{U U}^{T}=\mathbf{G G}^{T}+\mathbf{K} \mathbf{K}^{T}=\mathbf{I}$. Recognizing that the vectors $\mathbf{s}_{i}^{\perp} /\left\|\mathbf{s}_{i}^{\perp}\right\|(i=$ $\left.1,2, \ldots, M_{\mathrm{R}}-1\right)$ are obtained by applying the Gram-Schmidt procedure to the real-valued $\left(M_{\mathrm{R}}-1\right) \times M_{\mathrm{T}}$ i.i.d. Gaussian matrix $\left[\begin{array}{llll}\mathbf{s}_{1} & \mathbf{s}_{2} & \cdots & \mathbf{s}_{M_{\mathrm{R}}-1}\end{array}\right]^{T}$ with zero-mean entries, we can take the stacked matrix $\mathbf{U}=\left[\begin{array}{ll}\mathbf{G} & \mathbf{K}\end{array}\right]$ to be given by the Q-matrix obtained by applying the QRdecomposition to an $M_{\mathrm{T}} \times M_{\mathrm{T}}$ i.i.d. real-valued Gaussian matrix with zero-mean entries. Note that using the Gram-Schmidt procedure for QR-decomposition yields the unique factorization characterized by positive entries on the main diagonal of the R-matrix [43, Th. 2.6.1]. Next, realizing that

$$
\begin{aligned}
\left\|\mathbf{A}_{M_{\mathrm{R}}} \operatorname{Im}\left(\mathbf{s}_{M_{\mathrm{R}}}\right)\right\|^{2} & =\left(\operatorname{Im}\left(\mathbf{s}_{M_{\mathrm{R}}}\right)\right)^{T}\left(\sum_{n=M_{\mathrm{R}}}^{M_{\mathrm{T}}} \mathbf{u}_{n} \mathbf{u}_{n}^{T}\right) \operatorname{Im}\left(\mathbf{s}_{M_{\mathrm{R}}}\right) \\
& =M_{\mathrm{T}} M_{\mathrm{R}} \sum_{n=M_{\mathrm{R}}}^{M_{\mathrm{T}}}[\mathbf{U}]_{M_{\mathrm{R}}, n}^{2}
\end{aligned}
$$


the proof is complete upon deriving the pdf of $\sum_{n=M_{\mathrm{R}}}^{M_{\mathrm{T}}}[\mathbf{U}]_{M_{\mathrm{R}}, n}^{2}$. It is well known that, applying any procedure for $\mathrm{QR}$-decomposition leading to the unique factorization where the elements on the main diagonal of the R-matrix are positive, the resulting Q-matrix $\mathrm{Q}$ is distributed such that $\mathrm{AQB} \stackrel{\mathrm{d}}{=} \mathrm{Q}$ for any orthonormal $13 \mathrm{~A}$ and $\mathbf{B}[53$, Th. 3.2]. Choosing $\mathbf{A}$ and $\mathbf{B}$ to be permutation matrices, we can conclude that the rows and columns of $\mathbf{Q}$, and hence $\mathbf{U}$ in our case, are all equally distributed. Now, the quantity we are interested in is the sum of squares of the elements $\left\{M_{\mathrm{R}}, M_{\mathrm{R}}+1, \ldots, M_{\mathrm{T}}\right\}$ in any such row or column. Specifically, if the Gram-Schmidt procedure is used to obtain the QR-decomposition, the first column of $\mathbf{U}$ is given explicitly as $\mathbf{s}_{1} /\left\|\mathbf{s}_{1}\right\|$. From [14, Def. 1.4] we know that the quantities $\left[\mathbf{s}_{1}\right]_{n}^{2} /\left\|\mathbf{s}_{1}\right\|^{2}$ are jointly Dirichlet distributed, i.e.,

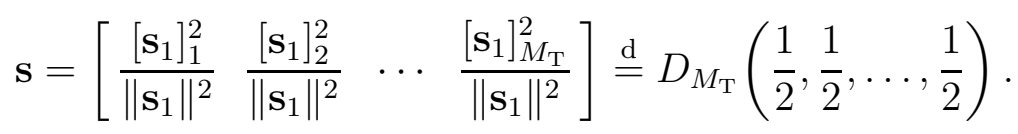

Partitioning $\mathbf{s}$ into subvectors of length $M_{\mathrm{T}}-M_{\mathrm{R}}+1$ and $M_{\mathrm{R}}-1$, respectively, and employing [14, Th. 1.4 and Th. 1.5] (reproduced as Theorem 10 and Theorem 11, respectively, in the Appendix for convenience), it follows that

$$
M_{\mathrm{T}} M_{\mathrm{R}} \sum_{n=M_{\mathrm{R}}}^{M_{\mathrm{T}}}[\mathrm{U}]_{M_{\mathrm{R}}, n}^{2} \stackrel{\mathrm{d}}{=} M_{\mathrm{T}} M_{\mathrm{R}} \beta\left(\frac{M_{\mathrm{T}}-M_{\mathrm{R}}+1}{2}, \frac{M_{\mathrm{R}}-1}{2}\right)
$$

where $\beta(a, b)$ is a beta-distributed RV with parameters $a$ and $b$ as defined in the Notations section.

Note that even though the results in (56) and Theorem 6 have a striking similarity and (56) provides an approximation for the exact result in Theorem 6, it seems difficult to derive (56) directly from Theorem 6 .

We are now ready to state an analytic lower bound on the ergodic capacity of an effective channel resulting from a rank-1 physical channel with unit-modulus entries subject to fully uncorrelated phase noise.

\footnotetext{
${ }^{13}$ The matrix $\mathbf{A}$ is said to be orthonormal if $\mathbf{A} \mathbf{A}^{T}=\mathbf{I}$.
} 
Theorem 7: For a separable timing matrix $\mathbf{T}$ and under the small phase noise approximation $\sigma_{\varphi}^{2} \ll 1$ so that $\widetilde{\boldsymbol{\Theta}}=\mathbf{1}+j \boldsymbol{\Phi}$ with $\operatorname{vec}(\boldsymbol{\Phi}) \stackrel{\mathrm{d}}{=} \mathcal{N}\left(\mathbf{0}, \sigma_{\varphi}^{2} \mathbf{I}_{M_{\mathrm{T}} M_{\mathrm{R}}}\right)$, assuming that $\mathbf{H}=\mathbf{g h}^{T}$ with $\left|[\mathbf{g}]_{i}\right|=1\left(i=1,2, \ldots, M_{\mathrm{R}}\right)$ and $\left|[\mathbf{h}]_{i}\right|=1\left(i=1,2, \ldots, M_{\mathrm{T}}\right)$, the ergodic capacity of the effective channel $\widehat{\mathbf{H}}$ satisfies

$$
\begin{array}{ll}
\widehat{C} \geq \log \left(1+\sum_{n=1}^{M_{\mathrm{R}}}\left(\frac{\rho}{M_{\mathrm{T}}}\right)^{n}\left(\begin{array}{c}
M_{\mathrm{R}} \\
n
\end{array}\right) \prod_{i=0}^{n-1}\left(\delta_{i} n M_{\mathrm{T}}+2 \sigma_{\varphi}^{2} e^{\Psi\left(\frac{M_{\mathrm{T}}-i}{2}\right)}\right)\right), \quad M_{\mathrm{R}} \leq M_{\mathrm{T}} \\
\widehat{C} \geq \log \left(1+\sum_{n=1}^{M_{\mathrm{T}}}\left(\frac{\rho}{M_{\mathrm{T}}}\right)^{n}\left(\begin{array}{c}
M_{\mathrm{T}} \\
n
\end{array}\right) \prod_{i=0}^{n-1}\left(\delta_{i} n M_{\mathrm{R}}+2 \sigma_{\varphi}^{2} e^{\Psi\left(\frac{M_{\mathrm{R}}-i}{2}\right)}\right)\right), & M_{\mathrm{R}}>M_{\mathrm{T}} .
\end{array}
$$

Proof: We provide the proof for $M_{\mathrm{R}} \leq M_{\mathrm{T}}$ only. The case $M_{\mathrm{R}}>M_{\mathrm{T}}$ follows exactly the same line of reasoning. We start by using Lemma 2 and noting that our assumptions imply that

$$
\begin{aligned}
\hat{I} & =\log \operatorname{det}\left(\mathbf{I}+\frac{\rho}{M_{\mathrm{T}}} \widehat{\mathbf{H}} \widehat{\mathbf{H}}^{H}\right)=\log \operatorname{det}\left(\mathbf{I}+\frac{\rho}{M_{\mathrm{T}}} \widetilde{\boldsymbol{\Theta}} \widetilde{\boldsymbol{\Theta}}^{H}\right) \\
& =\log \operatorname{det}\left(\mathbf{I}+\frac{\rho}{M_{\mathrm{T}}} \mathbf{S S}^{H}\right)
\end{aligned}
$$

where $\mathbf{S}=-j \boldsymbol{\Sigma}+\widetilde{\boldsymbol{\Phi}}$ was defined in the proof of Theorem 5. Next, using [35, Eq. (25)], it follows that

$$
\widehat{C} \geq \log \left(1+\sum_{i=1}^{M_{\mathrm{R}}}\left(\frac{\rho}{M_{\mathrm{T}}}\right)^{i} \sum_{l_{1}<l_{2}<\ldots<l_{i}} e^{\mathbb{E}\left\{\log _{e} \operatorname{det}\left(\left(\mathbf{S S}^{H}\right)_{l_{1}<l_{2}<\ldots<l_{i}}\right)\right\}}\right)
$$

where $\left(\mathbf{S S}^{H}\right)_{l_{1}<l_{2}<\ldots<l_{i}}$ denotes the submatrix of $\mathbf{S S}^{H}$ obtained by retaining the rows $l_{1}<$ $l_{2}<\ldots<l_{i}$ and the columns $l_{1}<l_{2}<\ldots<l_{i}$. The summation in (63) is over all ordered tuples $\left(l_{1}, l_{2}, \ldots, l_{i}\right)$ chosen from the set $\left\{1,2, \ldots, M_{\mathrm{R}}\right\}$. Next, we note that the pdf of $\operatorname{det}\left(\left(\mathbf{S S}^{H}\right)_{l_{1}<l_{2}<\ldots<l_{i}}\right)$ follows in a straightforward fashion from the results developed in the proof of Theorem 5, In particular, $\operatorname{det}\left(\left(\mathbf{S S}^{H}\right)_{l_{1}<l_{2}<\ldots<l_{i}}\right)$ is the determinant of the matrix $\widetilde{\mathbf{S S}}^{H}$ where the $i \times M_{\mathrm{T}}$ matrix $\widetilde{\mathbf{S}}$ is obtained from $\mathbf{S}$ by retaining the rows $\left\{l_{1}, l_{2}, \ldots, l_{i}\right\}$. Distinguishing between the terms, in the summation over $l_{1}<l_{2}<\ldots<l_{i}$ on the RHS of (63), that have 
$l_{1}=1$ and those where $l_{1}>1$, we obtain

$$
\mathbb{E}\left\{\log _{e} \operatorname{det}\left(\left(\mathbf{S S}^{H}\right)_{l_{1}<l_{2}<\ldots<l_{i}}\right)\right\} \stackrel{(a)}{\geq} \mathbb{E}\left\{\log _{e}\left(\left(\chi_{M_{\mathrm{T}}, \sigma_{\varphi}^{2}}^{2}+M_{\mathrm{T}} M_{\mathrm{R}}\right) \prod_{l=1}^{i-1} \chi_{M_{\mathrm{T}}-l, \sigma_{\varphi}^{2}}^{2}\right)\right\}
$$

in the former case and

$$
\mathbb{E}\left\{\log _{e} \operatorname{det}\left(\left(\mathbf{S S}^{H}\right)_{l_{1}<l_{2}<\ldots<l_{i}}\right)\right\}=\mathbb{E}\left\{\log _{e}\left(\prod_{l=0}^{i-1} \chi_{M_{\mathrm{T}}-l, \sigma_{\varphi}^{2}}^{2}\right)\right\}
$$

in the latter case, where (a) is obtained as follows. Recognizing that $\operatorname{det}\left(\left(\mathbf{S S}^{H}\right)_{l_{1}<l_{2}<\ldots<l_{i}}\right)=$ $\operatorname{det}\left(\left(\widetilde{\boldsymbol{\Theta}} \widetilde{\Theta}^{H}\right)_{l_{1}<l_{2}<\ldots<l_{i}}\right)$ and applying (56) properly modified to account for the fact that we are interested in the submatrix of $\widetilde{\Theta} \widetilde{\Theta}^{H}$ obtained by retaining the rows $\left\{l_{1}<l_{2}<\ldots<l_{i}\right\}$ in $\widetilde{\Theta}$ would yield an approximate expression for the left-hand side (LHS) in (64). However, using Theorem 5 and invoking Theorem 12 in the Appendix, we can show that the lower bound in (64) holds firmly. Specifically, starting from (50) and setting $\mathfrak{X}_{M_{\mathrm{T}}, \sigma_{\varphi}^{2}}^{\prime}=\mathbb{E}\left\{\log _{e}\left(M_{\mathrm{T}} M_{\mathrm{R}}+\chi_{M_{\mathrm{T}}, \sigma_{\varphi}^{2}}^{2}\right)\right\}$, for brevity, we can rewrite the LHS of (64) as

$$
\begin{aligned}
\mathbb{E}\left\{\log _{e} \operatorname{det}\left(\left(\mathbf{S S}^{H}\right)_{l_{1}<l_{2}<\ldots<l_{i}}\right)\right\} & =\mathfrak{X}_{M_{\mathrm{T}}, \sigma_{\varphi}^{2}}^{\prime}+\sum_{l=2}^{i} \mathbb{E}_{X_{l}, X_{1}, X_{2}, \eta^{(l)}}\left\{\log _{e}(\underbrace{\chi_{M_{\mathrm{T}}-l, \sigma_{\varphi}^{2}}^{2}}_{X_{l}}+Z\left(\eta^{(l)}\right))\right\} \\
& =\mathfrak{X}_{M_{\mathrm{T}}, \sigma_{\varphi}^{2}}^{\prime}+\sum_{l=2}^{i} \mathbb{E}_{X_{l}} \mathbb{E}_{\eta^{(l)}} \mathbb{E}_{X_{1}, X_{2} \mid X_{l}, \eta^{(l)}}\left\{\log _{e}\left(X_{l}+Z\left(\eta^{(l)}\right)\right)\right\} \\
& \stackrel{(a)}{\geq} \mathfrak{X}_{M_{\mathrm{T}}, \sigma_{\varphi}^{2}}^{\prime}+\sum_{l=2}^{i} \mathbb{E}_{X_{l}} \mathbb{E}_{Y \mid X_{l}}\left\{\log _{e}(X_{l}+\underbrace{\chi_{1, \sigma_{\varphi}^{2}}^{2}}_{Y})\right\} \\
& =\mathfrak{X}_{M_{\mathrm{T}}, \sigma_{\varphi}^{2}}^{\prime}+\sum_{l=1}^{i-1} \mathbb{E}\left\{\log _{e}\left(\chi_{M_{\mathrm{T}}-l, \sigma_{\varphi}^{2}}^{2}\right)\right\}
\end{aligned}
$$

where (a) follows from Theorem 12 in the Appendix. The relation in (65) is obtained in exactly the same fashion upon noting that the term $-j \sqrt{M_{\mathrm{T}} M_{\mathrm{R}}}$ is absent in the sets $\left(l_{1}, l_{2}, \ldots, l_{i}\right)$ where $l_{1}>1$. The number of terms in the first group (where $l_{1}=1$ ) is given by $\left(\begin{array}{c}M_{R}-1 \\ i-1\end{array}\right)$ whereas the number of terms in the second group is $\left(\begin{array}{c}M_{\mathrm{R}} \\ i\end{array}\right)-\left(\begin{array}{c}M_{\mathrm{R}}-1 \\ i-1\end{array}\right)$. It remains to find analytic 
expressions for the RHS of (64) and of (65). It is well known [54] that $\mathbb{E}\left\{\log _{e}\left(\chi_{n, \sigma_{\varphi}^{2}}^{2}\right)\right\}=$ $\log _{e}\left(2 \sigma_{\varphi}^{2}\right)+\Psi(n / 2) \triangleq \mathfrak{X}_{n, \sigma_{\varphi}^{2}}$. The term $\mathfrak{X}_{M_{\mathrm{T}}, \sigma_{\varphi}^{2}}^{\prime}=\mathbb{E}\left\{\log _{e}\left(\chi_{M_{\mathrm{T}}, \sigma_{\varphi}^{2}}^{2}+M_{\mathrm{T}} M_{\mathrm{R}}\right)\right\}$ has a closed-form analytic expression in terms of the generalized exponential integral $E_{\nu}(z)=\int_{1}^{\infty} t^{-\nu} e^{-z t} \mathrm{~d} t$, $\operatorname{Re}(z)>0$. For our purposes, we shall, however, be content with a simple lower bound obtained by applying Jensen's inequality to the function $f(x)=\log _{e}\left(e^{x}+a\right)$, which results in

$$
\begin{aligned}
\mathfrak{X}_{M_{\mathrm{T}}, \sigma_{\varphi}^{2}}^{\prime} & \geq \log _{e}\left(e^{\mathbb{E}\left\{\log _{e}\left(\chi_{M_{\mathrm{T}}, \sigma_{\varphi}^{2}}^{2}\right)\right\}}+M_{\mathrm{T}} M_{\mathrm{R}}\right) \\
& =\log _{e}\left(e^{\mathfrak{X}_{M_{\mathrm{T}}, \sigma_{\varphi}^{2}}}+M_{\mathrm{T}} M_{\mathrm{R}}\right) .
\end{aligned}
$$

Putting the pieces together, we get

$$
\begin{aligned}
e^{\mathbb{E}\left\{\log _{e} \operatorname{det}\left(\left(\mathbf{S S}^{H}\right)_{l_{1}<l_{2}<\ldots<l_{i}}\right)\right\}} & \geq\left(M_{\mathrm{T}} M_{\mathrm{R}}+e^{\mathfrak{X}_{M_{\mathrm{T}}, \sigma_{\varphi}^{2}}}\right) e^{\sum_{l=1}^{i-1} \mathfrak{X}_{M_{\mathrm{T}}-l, \sigma_{\varphi}^{2}}} \\
& =M_{\mathrm{T}} M_{\mathrm{R}} e^{\sum_{l=1}^{i-1} \mathfrak{X}_{M_{\mathrm{T}}-l, \sigma_{\varphi}^{2}}}+e^{\sum_{l=0}^{i-1} \mathfrak{X}_{M_{\mathrm{T}}-l, \sigma_{\varphi}^{2}}}
\end{aligned}
$$

for 64 and

$$
\left.\left.e^{\mathbb{E}\left\{\operatorname { l o g } _ { e } \operatorname { d e t } \left(\left(\mathbf{S S}^{H}\right) l_{1}<l_{2}<\ldots<l_{i}\right.\right.}\right)\right\}=e^{\sum_{l=0}^{i-1} \mathfrak{X}_{M_{\mathrm{T}}-l, \sigma_{\varphi}^{2}}}
$$

for (65). Combining our results and noting that the term $\exp \left(\sum_{l=0}^{i-1} \mathfrak{X}_{M_{\mathrm{T}}-l, \sigma_{\varphi}^{2}}\right)$ occurs in both cases so that its total number of occurences is $\left(\begin{array}{c}M_{\mathrm{R}} \\ i\end{array}\right)$, finally yields

$$
\begin{aligned}
\left.\left.\sum_{l_{1}<l_{2}<\ldots<l_{i}} e^{\mathbb{E}\left\{\operatorname { l o g } _ { e } \operatorname { d e t } \left(\left(\mathbf{S S}^{H}\right) l_{1}<l_{2}<\ldots<l_{i}\right.\right.}\right)\right\} \\
\quad \geq\left(\begin{array}{c}
M_{\mathrm{R}}-1 \\
i-1
\end{array}\right) M_{\mathrm{T}} M_{\mathrm{R}} e^{\sum_{n=1}^{i-1} \mathfrak{X}_{M_{\mathrm{T}}-n, \sigma_{\varphi}^{2}}}+\left(\begin{array}{c}
M_{\mathrm{R}} \\
i
\end{array}\right) e^{\sum_{n=0}^{i-1} \mathfrak{X}_{M_{\mathrm{T}}-n, \sigma_{\varphi}}}
\end{aligned}
$$

which, upon inserting into (63) and reorganizing terms, concludes the proof.

The result in (61) can be made more explicit by using the simplifications for the digamma function at positive integer multiples of $1 / 2$ given by (3). Furthermore, we note that Theorem 7 can be generalized to the cases where i) $\left|[\mathbf{h}]_{i}\right|=1, \forall i, \mathrm{~g}$ is general and $M_{\mathrm{R}} \leq M_{\mathrm{T}}$ and ii) $\left|[\mathbf{g}]_{i}\right|=1, \forall i, \mathbf{h}$ is general and $M_{\mathrm{R}}>M_{\mathrm{T}}$. The corresponding results are stated, without proof, 


$$
\begin{aligned}
\text { i) } \quad \widehat{C} \geq \log \left(1+\sum_{n=1}^{M_{\mathrm{R}}}\left(\frac{\rho}{M_{\mathrm{T}}}\right)^{n} K_{n}(\mathbf{g}) \prod_{i=0}^{n-1}\left(\delta_{i} n M_{\mathrm{T}}+2 \sigma_{\varphi}^{2} e^{\Psi\left(\frac{M_{\mathrm{T}}-i}{2}\right)}\right)\right), \quad M_{\mathrm{R}} \leq M_{\mathrm{T}} \\
\text { ii) } \widehat{C} \geq \log \left(1+\sum_{n=1}^{M_{\mathrm{T}}}\left(\frac{\rho}{M_{\mathrm{T}}}\right)^{n} K_{n}(\mathbf{h}) \prod_{i=0}^{n-1}\left(\delta_{i} n M_{\mathrm{R}}+2 \sigma_{\varphi}^{2} e^{\Psi\left(\frac{M_{\mathrm{R}}-i}{2}\right)}\right)\right), \quad M_{\mathrm{R}}>M_{\mathrm{T}}
\end{aligned}
$$

where $K_{n}(\mathbf{x})=\sum_{\mathbf{s} \in \mathcal{S}_{n, l(\mathbf{x})}} \prod_{i=1}^{n}\left|[\mathbf{x}]_{s_{i}}\right|^{2}, \mathcal{S}_{k, m}$ is the set of all possible ordered $k$-tuples $\mathbf{s}=$ $\left(s_{1}, s_{2}, \ldots, s_{k}\right)$ with $1 \leq s_{1}<s_{2}<\ldots<s_{k} \leq m$, and $l(\mathbf{x})$ is the number of elements in the vector $\mathbf{x}$.

Again assuming $\left|[\mathbf{g}]_{i}\right|=1\left(i=1,2, \ldots, M_{\mathrm{R}}\right)$ and $\left|[\mathbf{h}]_{i}\right|=1\left(i=1,2, \ldots, M_{\mathrm{T}}\right)$, further lowerbounding (61) by ignoring the first term inside the "log" and retaining only the highest-order (in $\rho$ ) term yields

$$
\begin{array}{ll}
\widehat{C} \geq M_{\mathrm{R}} \log \left(\frac{\rho}{M_{\mathrm{T}}}\right)+\log \left(\prod_{i=0}^{M_{\mathrm{R}}-1}\left(\delta_{i} M_{\mathrm{T}} M_{\mathrm{R}}+2 \sigma_{\varphi}^{2} e^{\Psi\left(\frac{M_{\mathrm{T}}-i}{2}\right)}\right)\right), \quad M_{\mathrm{R}} \leq M_{\mathrm{T}} \\
\widehat{C} \geq M_{\mathrm{T}} \log \left(\frac{\rho}{M_{\mathrm{T}}}\right)+\log \left(\prod_{i=0}^{M_{\mathrm{T}}-1}\left(\delta_{i} M_{\mathrm{T}} M_{\mathrm{R}}+2 \sigma_{\varphi}^{2} e^{\Psi\left(\frac{M_{\mathrm{R}}-i}{2}\right)}\right)\right), \quad M_{\mathrm{R}}>M_{\mathrm{T}}
\end{array}
$$

which clearly shows that the effective channel has full rank and hence its multiplexing gain is given by $\min \left(M_{\mathrm{T}}, M_{\mathrm{R}}\right)$. Put differently, phase noise can cause a rank-1 physical channel to appear like a full-rank channel. In Section VII, we shall show, based on measurement results, that significant rank increase does, indeed, occur in practice.

We shall next provide a slightly looser (than (61) lower bound on $\widehat{C}$ with a simpler structure.

Theorem 8: For a separable timing matrix $\mathbf{T}$ and under the small phase noise approximation $\sigma_{\varphi}^{2} \ll 1$ so that $\widetilde{\boldsymbol{\Theta}}=\mathbf{1}+j \boldsymbol{\Phi}$ with $\operatorname{vec}(\boldsymbol{\Phi}) \stackrel{\mathrm{d}}{=} \mathcal{N}\left(\mathbf{0}, \sigma_{\varphi}^{2} \mathbf{I}_{M_{\mathrm{T}} M_{\mathrm{R}}}\right)$, assuming that $\mathbf{H}=\mathbf{g h}^{T}$ with $\left|[\mathbf{g}]_{i}\right|=1\left(i=1,2, \ldots, M_{\mathrm{R}}\right)$ and $\left|[\mathbf{h}]_{i}\right|=1\left(i=1,2, \ldots, M_{\mathrm{T}}\right)$, the ergodic capacity of the 
effective channel $\widehat{\mathbf{H}}$ satisfies

$$
\begin{array}{ll}
\widehat{C} \geq \sum_{i=0}^{M_{\mathrm{R}}-1} \log \left(1+\frac{\rho}{M_{\mathrm{T}}}\left(M_{\mathrm{T}} M_{\mathrm{R}} \delta_{i}+2 \sigma_{\varphi}^{2} e^{\Psi\left(\frac{M_{\mathrm{T}}-i}{2}\right)}\right)\right), & M_{\mathrm{R}} \leq M_{\mathrm{T}} \\
\widehat{C} \geq \sum_{i=0}^{M_{\mathrm{T}}-1} \log \left(1+\frac{\rho}{M_{\mathrm{T}}}\left(M_{\mathrm{T}} M_{\mathrm{R}} \delta_{i}+2 \sigma_{\varphi}^{2} e^{\Psi\left(\frac{M_{\mathrm{R}}-i}{2}\right)}\right)\right), & M_{\mathrm{R}}>M_{\mathrm{T}} .
\end{array}
$$

Proof: We provide the proof for $M_{\mathrm{R}} \leq M_{\mathrm{T}}$ only. The case $M_{\mathrm{R}}>M_{\mathrm{T}}$ follows exactly the same line of reasoning. We start by noting that (64) and (65) can be combined as

$$
\mathbb{E}\left\{\log _{e} \operatorname{det}\left(\left(\mathbf{S S}^{H}\right)_{l_{1}<l_{2}<\ldots<l_{i}}\right)\right\} \geq \sum_{l=0}^{i-1} \mathbb{E}\left\{\log _{e}\left(M_{\mathrm{T}} M_{\mathrm{R}} \delta_{l} \delta_{l_{1}-1}+\chi_{M_{\mathrm{T}}-l, \sigma_{\varphi}^{2}}^{2}\right)\right\}
$$

which, upon inserting into (63), yields

$$
\widehat{C} \geq \log \left(1+\sum_{i=1}^{M_{\mathrm{R}}}\left(\frac{\rho}{M_{\mathrm{T}}}\right)^{i} \sum_{l_{1}<l_{2}<\ldots<l_{i}} \prod_{l=0}^{i-1} e^{\mathbb{E}\left\{\log _{e}\left(M_{\mathrm{T}} M_{\mathrm{R}} \delta_{l} \delta_{l_{1}-1}+\chi_{M_{\mathrm{T}}-l, \sigma_{\varphi}^{2}}^{2}\right)\right\}}\right)=C_{2} .
$$

The proof will be completed by showing that $2^{C_{2}} \geq 2^{C_{1}}$ with

$$
C_{1}=\log \prod_{i=0}^{M_{\mathrm{R}}-1}\left(1+\frac{\rho}{M_{\mathrm{T}}} e^{\mathbb{E}\left\{\log _{e}\left(M_{\mathrm{T}} M_{\mathrm{R}} \delta_{i}+\chi_{M_{\mathrm{T}}-i, \sigma_{\varphi}^{2}}^{2}\right)\right\}}\right)
$$

and noting that the RHS of (69) is obtained by lower-bounding the term corresponding to $i=0$ in (70) according to (67). Setting $X_{n}=\mathfrak{X}_{M_{\mathrm{T}}-n, \sigma_{\varphi}^{2}}\left(n=0,1, \ldots, M_{\mathrm{R}}-1\right)$ and $X_{0}^{\prime}=$ $\mathbb{E}\left\{\log _{e}\left(M_{\mathrm{T}} M_{\mathrm{R}}+\chi_{M_{\mathrm{T}}, \sigma_{\varphi}^{2}}^{2}\right)\right\}$, and expanding

$$
2^{C_{1}}=\left(1+\frac{\rho}{M_{\mathrm{T}}} e^{X_{0}^{\prime}}\right) \prod_{i=1}^{M_{\mathrm{R}}-1}\left(1+\frac{\rho}{M_{\mathrm{T}}} e^{X_{i}}\right)
$$


we get

2a)

$$
\begin{aligned}
2^{C_{1}}=1 & +\left(\frac{\rho}{M_{\mathrm{T}}}\right)\left(e^{X_{0}^{\prime}}+e^{X_{1}}+\cdots+e^{X_{M_{\mathrm{R}}-1}}\right) \\
& +\left(\frac{\rho}{M_{\mathrm{T}}}\right)^{2}\left(e^{X_{0}^{\prime}} e^{X_{1}}+e^{X_{0}^{\prime}} e^{X_{2}}+\cdots+e^{X_{0}^{\prime}} e^{X_{M_{\mathrm{R}}-1}}\right.
\end{aligned}
$$

$$
\left.+e^{X_{1}} e^{X_{2}}+e^{X_{1}} e^{X_{3}}+\cdots+\cdots+e^{X_{M_{\mathrm{R}}-2}} e^{X_{M_{\mathrm{R}}-1}}\right)
$$

3a)

$$
\begin{array}{r}
+\left(\frac{\rho}{M_{\mathrm{T}}}\right)^{3}\left(e^{X_{0}^{\prime}} e^{X_{1}} e^{X_{2}}+e^{X_{0}^{\prime}} e^{X_{1}} e^{X_{3}}+\cdots+e^{X_{0}^{\prime}} e^{X_{M_{\mathrm{R}}-2}} e^{X_{M_{\mathrm{R}}-1}}\right. \\
\left.+e^{X_{1}} e^{X_{2}} e^{X_{3}}+\cdots+\ldots+e^{X_{M_{\mathrm{R}}-3}} e^{X_{M_{\mathrm{R}}-2}} e^{X_{M_{\mathrm{R}}-1}}\right)
\end{array}
$$

3b)

$$
\begin{array}{cc}
+ & \vdots \\
+\left(\frac{\rho}{M_{\mathrm{T}}}\right)^{M_{\mathrm{R}}} & \left(e^{X_{0}^{\prime}} e^{X_{1}} \cdots e^{X_{M_{\mathrm{R}}-1}}\right)
\end{array}
$$

where lines $2 \mathrm{a})$ and $2 \mathrm{~b}$ ) contain $\left(\begin{array}{c}M_{\mathrm{R}}-1 \\ 1\end{array}\right)$ and $\left(\begin{array}{c}M_{\mathrm{R}}-1 \\ 2\end{array}\right)$ terms, respectively, and lines $\left.3 \mathrm{a}\right)$ and $3 \mathrm{~b}$ ) contain $\left(\begin{array}{c}M_{\mathrm{R}}-1 \\ 2\end{array}\right)$ and $\left(\begin{array}{c}M_{\mathrm{R}}-1 \\ 3\end{array}\right)$ terms, respectively. Expanding

$$
2^{C_{2}}=1+\sum_{i=1}^{M_{\mathrm{R}}}\left(\frac{\rho}{M_{\mathrm{T}}}\right)^{i}\left(\sum_{1=l_{1}<l_{2}<\ldots<l_{i}}\left(X_{0}^{\prime} \prod_{l=1}^{i-1} e^{X_{l}}\right)+\sum_{1<l_{1}<l_{2}<\ldots<l_{i}}\left(X_{0} \prod_{l=1}^{i-1} e^{X_{l}}\right)\right)
$$

we obtain

2a)

$$
2^{C_{2}}=1+\left(\frac{\rho}{M_{\mathrm{T}}}\right)\left(e^{X_{0}^{\prime}}+e^{X_{0}}+\cdots+e^{X_{0}}\right)
$$

$+\left(\frac{\rho}{M_{\mathrm{T}}}\right)^{2}\left(e^{X_{0}^{\prime}} e^{X_{1}}+e^{X_{0}^{\prime}} e^{X_{1}}+\cdots+e^{X_{0}^{\prime}} e^{X_{1}}\right.$

2b)

$$
\left.+e^{X_{0}} e^{X_{1}}+e^{X_{0}} e^{X_{1}}+\cdots+\cdots+e^{X_{0}} e^{X_{1}}\right)
$$

$$
+\left(\frac{\rho}{M_{\mathrm{T}}}\right)^{3}\left(e^{X_{0}^{\prime}} e^{X_{1}} e^{X_{2}}+e^{X_{0}^{\prime}} e^{X_{1}} e^{X_{2}}+\cdots+e^{X_{0}^{\prime}} e^{X_{1}} e^{X_{2}}\right.
$$

3b)

$$
\left.+e^{X_{0}} e^{X_{1}} e^{X_{2}}+e^{X_{0}} e^{X_{1}} e^{X_{2}}+\cdots+\cdots+e^{X_{0}} e^{X_{1}} e^{X_{2}}\right)
$$

$$
\begin{array}{cc}
+ & \vdots \\
+\left(\frac{\rho}{M_{\mathrm{T}}}\right)^{M_{\mathrm{R}}} & \left(e^{X_{0}^{\prime}} e^{X_{1}} \cdots e^{X_{M_{\mathrm{R}}-1}}\right)
\end{array}
$$


where the number of terms in lines $2 a), 2 b), 3 a$ ), and $3 b$ ) is the same as in the corresponding lines in the expansion of $2^{C_{1}}$. In both cases, the number of terms associated with the factor $\left(\rho / M_{\mathrm{T}}\right)^{i}$ is given by $\left(\begin{array}{c}M_{\mathrm{R}} \\ i\end{array}\right)$. The proof is completed by comparing (71) and (72) term by term and noting that the monotonicity of the digamma function implies $\mathfrak{X}_{M_{\mathrm{T}}-n, \sigma_{\varphi}^{2}} \geq \mathfrak{X}_{M_{\mathrm{T}}-n-k, \sigma_{\varphi}^{2}}$ and hence $X_{n} \geq X_{n+k}$ for $k \geq 1$.

So far we derived lower bounds on $\widehat{C}$. We shall next show that the result in Theorem 5 together with a technique first proposed in [35] (and used to derive the lower bound in Theorem 7) can be employed to derive a tight analytic upper bound on $\widehat{C}$.

Theorem 9: Under the assumptions in Theorem 8 , the ergodic capacity of the effective MIMO channel can be upper-bounded as

$$
\widehat{C} \leq \log \left(1+\sum_{n=1}^{M_{\mathrm{R}}}\left(\frac{\rho}{M_{\mathrm{T}}}\right)^{n}\left(\sigma_{\varphi}^{2}\right)^{n}\left(\begin{array}{c}
M_{\mathrm{R}} \\
n
\end{array}\right)\left(\begin{array}{c}
M_{\mathrm{T}} \\
n
\end{array}\right) n !\left(1+\frac{n}{\sigma_{\varphi}^{2}}\right)\right)
$$

Proof: We provide the proof for $M_{\mathrm{R}} \leq M_{\mathrm{T}}$ only. The case $M_{\mathrm{R}}>M_{\mathrm{T}}$ follows exactly the same line of reasoning. The proof starts from [35, Eq. (19)] which, specialized to our case, reads

$$
\widehat{C} \leq \log \left(1+\sum_{n=1}^{M_{\mathrm{R}}}\left(\frac{\rho}{M_{\mathrm{T}}}\right)^{n} \sum_{l_{1}<l_{2}<\ldots<l_{n}} \mathbb{E}\left\{\operatorname{det}\left(\left(\mathbf{S S}^{H}\right)_{l_{1}<l_{2}<\ldots<l_{n}}\right)\right\}\right)
$$

where $\mathbf{S}$ was defined in the proof of Theorem 5 . The main point of the proof is to recognize that we can obtain analytic expressions for the terms $\mathbb{E}\left\{\operatorname{det}\left(\left(\mathbf{S S}^{H}\right)_{l_{1}<l_{2}<\ldots<l_{n}}\right)\right\}$ using (52). As already shown in the proof of Theorem 7, the terms in $\sum_{l_{1}<l_{2}<\ldots<l_{n}} \mathbb{E}\{\cdot\}$ on the RHS of (74) fall into two groups depending on whether $l_{1}=1$ or $l_{1}>1$. Specifically, for $l_{1}=1$ we have (cf. (66))

$$
\begin{aligned}
\mathbb{E}\left\{\operatorname{det}\left(\left(\mathbf{S S}^{H}\right)_{l_{1}<l_{2}<\ldots<l_{n}}\right)\right\}=M_{\mathrm{T}} M_{\mathrm{R}} & \prod_{i=2}^{n} \mathbb{E}\left\{\chi_{M_{\mathrm{T}}-i, \sigma_{\varphi}^{2}}^{2}+Z\left(\eta^{(i)}\right)\right\} \\
& +\mathbb{E}\left\{\chi_{M_{\mathrm{T}}, \sigma_{\varphi}^{2}}^{2}\right\} \prod_{i=2}^{n} \mathbb{E}\left\{\chi_{M_{\mathrm{T}}-i, \sigma_{\varphi}^{2}}^{2}+Z\left(\eta^{(i)}\right)\right\}
\end{aligned}
$$


and for $l_{1}>1$ (cf. (65) $)$

$$
\mathbb{E}\left\{\operatorname{det}\left(\left(\mathbf{S S}^{H}\right)_{l_{1}<l_{2}<\ldots<l_{n}}\right)\right\}=\mathbb{E}\left\{\chi_{M_{\mathrm{T}}, \sigma_{\varphi}^{2}}^{2}\right\} \prod_{i=2}^{n} \mathbb{E}\left\{\chi_{M_{\mathrm{T}}-i, \sigma_{\varphi}^{2}}^{2}+Z\left(\eta^{(i)}\right)\right\}
$$

Using $\mathbb{E}\left\{\chi_{M_{\mathrm{T}}-i, \sigma_{\varphi}^{2}}^{2}\right\}=\left(M_{\mathrm{T}}-i\right) \sigma_{\varphi}^{2}$, noting that

$$
\begin{aligned}
\mathbb{E}\left\{Z\left(\eta^{(i)}\right)\right\} & =\mathbb{E}_{\eta^{(i)}}\left\{\mathbb{E}\left\{\chi_{1, \sigma_{\varphi}^{2} \eta^{(i)}}^{2}+\chi_{1, \sigma_{\varphi}^{2}\left(1-\eta^{(i)}\right)}^{2} \mid \eta^{(i)}\right\}\right\} \\
& =\mathbb{E}_{\eta^{(i)}}\left\{\sigma_{\varphi}^{2} \eta^{(i)}+\sigma_{\varphi}^{2}\left(1-\eta^{(i)}\right)\right\}=\mathbb{E}_{\eta^{(i)}}\left\{\sigma_{\varphi}^{2}\right\}=\sigma_{\varphi}^{2}
\end{aligned}
$$

and counting the multiplicity of the terms as in (68), we obtain

$$
\begin{aligned}
& \sum_{l_{1}<l_{2}<\ldots<l_{n}} \mathbb{E}\left\{\operatorname{det}\left(\left(\mathbf{S S}^{H}\right)_{l_{1}<l_{2}<\ldots<l_{n}}\right)\right\} \\
& =\left(\begin{array}{c}
M_{\mathrm{R}}-1 \\
n-1
\end{array}\right) M_{\mathrm{T}} M_{\mathrm{R}} \prod_{i=2}^{n}\left(\left(M_{\mathrm{T}}-i+1\right) \sigma_{\varphi}^{2}\right)+\left(\begin{array}{c}
M_{\mathrm{R}} \\
n
\end{array}\right) M_{\mathrm{T}} \sigma_{\varphi}^{2} \prod_{i=2}^{n}\left(\left(M_{\mathrm{T}}-i+1\right) \sigma_{\varphi}^{2}\right) \\
& =\left(\begin{array}{c}
M_{\mathrm{R}}-1 \\
n-1
\end{array}\right) M_{\mathrm{T}} M_{\mathrm{R}} \frac{\left(M_{\mathrm{T}}-1\right) !}{\left(M_{\mathrm{T}}-n\right) !}\left(\sigma_{\varphi}^{2}\right)^{n-1}+\left(\begin{array}{c}
M_{\mathrm{R}} \\
n
\end{array}\right) M_{\mathrm{T}} \frac{\left(M_{\mathrm{T}}-1\right) !}{\left(M_{\mathrm{T}}-n\right) !}\left(\sigma_{\varphi}^{2}\right)^{n} \\
& \quad=\left(\begin{array}{c}
M_{\mathrm{R}} \\
n
\end{array}\right) \frac{n M_{\mathrm{T}} !}{\left(M_{\mathrm{T}}-n\right) !}\left(\sigma_{\varphi}^{2}\right)^{n-1}+\left(\begin{array}{c}
M_{\mathrm{R}} \\
n
\end{array}\right) \frac{M_{\mathrm{T}} !}{\left(M_{\mathrm{T}}-n\right) !}\left(\sigma_{\varphi}^{2}\right)^{n} .
\end{aligned}
$$

Putting the pieces together, we get (73), which concludes the proof.

We note that the proof of Theorem 9 can alternatively be carried out by obtaining analytic expressions for the terms $\mathbb{E}\left\{\operatorname{det}\left(\left(\mathbf{S S}^{H}\right)_{l_{1}<l_{2}<\ldots<l_{n}}\right)\right\}$ using properly modified versions of (59).

Numerical results: We shall next provide a numerical example that serves to quantify the quality of the lower bounds in Theorems 7 and 8 , and the upper bound in Theorem 9 . For a $4 \times 4$ deterministic physical channel $\mathbf{H}=\mathbf{g h}^{T}$ with $\left|[\mathbf{g}]_{i}\right|=1, \forall i$, and $\left|[\mathbf{h}]_{i}\right|=1, \forall i$, subject to $7^{\circ} \mathrm{rms}$ fully uncorrelated phase noise, Fig. 7 shows that the (ergodic) capacity of the effective channel starts deviating from the capacity of the rank-1 physical MIMO channel at $\rho \approx 15 \mathrm{~dB}$, and that significant capacity estimation errors (up to around 100\%) occur in the high-SNR regime. This behavior is consistent with our observation that the low-SNR capacity is not influenced by 
phase noise. Moreover, we observe that the lower and upper bounds (61) and (73), respectively, very accurately predict the capacity behavior of the effective channel.

High-SNR Variance of MI: Considering an ergodic block-fading MIMO channel, it was shown in [33] that the (high-SNR) variance of MI can be interpreted as quantifying the amount of "spatial averaging" that occurs on a per-stream basis in each fading block. The smaller the variance of MI the more spatial averaging occurs. As shown in [33], [55], $\sigma_{I}^{2}=\operatorname{Var}\{I\}$ for fixed $M_{\mathrm{R}}$, as a function of $M_{\mathrm{T}}$, has its maximum at $M_{\mathrm{T}}=M_{\mathrm{R}}$. For more details on the interpretation of $\sigma_{I}^{2}$ as a measure of the amount of spatial diversity, the interested reader is referred to [33]. We have seen that phase noise (and frequency offset) can have a significant impact on the rank of the MIMO channel and hence its spatial multiplexing gain. In the following, we shall characterize the increase in spatial diversity due to phase noise by analyzing the variance of the high-SNR MI of the effective MIMO channel. Finding exact expressions for $\sigma_{\hat{I}}^{2}=\operatorname{Var}\{\hat{I}\}$ seems difficult. Under the assumptions in Theorem 8 , we can, however, provide accurate and analytically tractable approximations, which are obtained as follows. Considering, for simplicity, the case $M_{\mathrm{R}} \leq M_{\mathrm{T}}$, we can infer from (62) and (56) that in the high-SNR regime

$$
\begin{aligned}
\hat{I} & \approx \log \left(\frac{\rho}{M_{\mathrm{T}}}\left(\chi_{M_{\mathrm{T}}, \sigma_{\varphi}^{2}}^{2}+M_{\mathrm{T}} M_{\mathrm{R}}\right) \prod_{i=1}^{M_{\mathrm{R}}-1} \chi_{M_{\mathrm{T}}-i, \sigma_{\varphi}^{2}}^{2}\right) \\
& =\log \left(\frac{\rho}{M_{\mathrm{T}}}\right)+\log \left(M_{\mathrm{T}} M_{\mathrm{R}}+\chi_{M_{\mathrm{T}}, \sigma_{\varphi}^{2}}^{2}\right)+\sum_{i=1}^{M_{\mathrm{R}}-1} \log \left(\chi_{M_{\mathrm{T}}-i, \sigma_{\varphi}^{2}}^{2}\right) .
\end{aligned}
$$

Writing the second term in (75) as

$$
\log \left(M_{\mathrm{T}} M_{\mathrm{R}}+\chi_{M_{\mathrm{T}}, \sigma_{\varphi}^{2}}^{2}\right)=\log \left(M_{\mathrm{T}} M_{\mathrm{R}}\right)+\log \left(1+\frac{\chi_{M_{\mathrm{T}}, \sigma_{\varphi}^{2}}^{2}}{M_{\mathrm{T}} M_{\mathrm{R}}}\right)
$$

and noting that for $M_{\mathrm{T}} M_{\mathrm{R}}$ large and $\sigma_{\varphi}^{2}$ small, we have

$$
\log \left(1+\frac{\chi_{M_{\mathrm{T}}, \sigma_{\varphi}^{2}}^{2}}{M_{\mathrm{T}} M_{\mathrm{R}}}\right) \approx \log (e) \frac{\chi_{M_{\mathrm{T}}, \sigma_{\varphi}^{2}}^{2}}{M_{\mathrm{T}} M_{\mathrm{R}}}
$$


it follows that

$$
\operatorname{Var}\{\hat{I}\} \approx \operatorname{Var}\left\{\log (e) \frac{\chi_{M_{\mathrm{T}}, \sigma_{\varphi}^{2}}^{2}}{M_{\mathrm{T}} M_{\mathrm{R}}}+\sum_{i=1}^{M_{\mathrm{R}}-1} \log \left(\chi_{M_{\mathrm{T}}-i, \sigma_{\varphi}^{2}}^{2}\right)\right\}
$$

Using

$$
\begin{aligned}
\operatorname{Var}\left\{\log _{e}\left(\chi_{M_{\mathrm{T}}-i, \sigma_{\varphi}^{2}}^{2}\right)\right\} & =\operatorname{Var}\left\{\log _{e}\left(\sigma_{\varphi}^{2}\right)+\log _{e}\left(\chi_{M_{\mathrm{T}}-i, 1}^{2}\right)\right\} \\
& =\operatorname{Var}\left\{\log _{e}\left(\chi_{M_{\mathrm{T}}-i, 1}^{2}\right)\right\}=\Psi^{\prime}\left(\frac{M_{\mathrm{T}}-i}{2}\right)
\end{aligned}
$$

and [54, App. A.7], we finally get

$$
\operatorname{Var}\{\hat{I}\} \approx(\log (e))^{2}\left(\frac{2 \sigma_{\varphi}^{4}}{M_{\mathrm{T}} M_{\mathrm{R}}^{2}}+\sum_{i=1}^{M_{\mathrm{R}}-1} \sum_{p=1}^{\infty} \frac{1}{\left(p+\frac{M_{\mathrm{T}}-i}{2}-1\right)^{2}}\right)
$$

Comparing (76) to the expression [33, Eq. (31)] for the variance of the high-SNR MI of an i.i.d. $M_{\mathrm{R}} \times M_{\mathrm{T}}$ complex Gaussian channel, we can show that, for the same number of transmit and receive antennas, the variance of the high-SNR MI of a rank-1 physical channel as in Theorem 8 subject to fully uncorrelated phase noise is higher than that in the i.i.d. complex Gaussian case, i.e.,

$$
\frac{2 \sigma_{\varphi}^{4}}{M_{\mathrm{T}} M_{\mathrm{R}}^{2}}+\sum_{i=1}^{M_{\mathrm{R}}-1} \Psi^{\prime}\left(\frac{M_{\mathrm{T}}-i}{2}\right) \geq \sum_{i=1}^{M_{\mathrm{R}}} \Psi^{\prime}\left(M_{\mathrm{T}}-i+1\right) .
$$

To prove (77), we omit the first term on the LHS and use Lemma 4 in the Appendix which leaves us with having to show that

$$
2 \sum_{i=1}^{M_{\mathrm{R}}-1} \Psi^{\prime}\left(M_{\mathrm{T}}-i\right) \geq \sum_{i=0}^{M_{\mathrm{R}}-1} \Psi^{\prime}\left(M_{\mathrm{T}}-i\right)
$$

Subtracting the common terms on both sides, it follows that (78) is equivalent to

$$
\sum_{i=1}^{M_{\mathrm{R}}-1} \Psi^{\prime}\left(M_{\mathrm{T}}-i\right) \geq \Psi^{\prime}\left(M_{\mathrm{T}}\right)
$$


The final result follows from the monotonicity property $\Psi^{\prime}\left(M_{\mathrm{T}}-i\right) \geq \Psi^{\prime}\left(M_{\mathrm{T}}\right), i \geq 1$. We note that (78) suggests that the variance of MI in the phase noise case is essentially twice that obtained for an i.i.d. Gaussian channel with the same number of transmit and receive antennas. The underlying reason lies in the fact that the individual chi-square terms in the phase noise case (cf. (64) and (65) ) have half the number of degrees of freedom when compared to the Gaussian channel case. The following numerical example corroborates the factor-2 statement motivated by the inequality (78).

Numerical result: For a deterministic rank-1 physical channel $\mathbf{H}=\mathbf{g h}^{T}$ with $\left|[\mathbf{g}]_{i}\right|=1, \forall i$, and $\left|[\mathbf{h}]_{i}\right|=1, \forall i$, subject to $3.5^{\circ} \mathrm{rms}$ fully uncorrelated phase noise, Fig. 8 shows $\sigma_{\hat{I}}^{2}$ according to the approximation (76) along with the exact result (obtained from Monte Carlo simulation) for $M_{\mathrm{R}}=10$ as a function of $M_{\mathrm{T}}$. We can see that the approximation is very tight for $M_{\mathrm{T}}>M_{\mathrm{R}}$ and $M_{\mathrm{T}}<M_{\mathrm{R}}$ and predicts the location of the maximum of $\sigma_{\hat{I}}^{2}$ accurately. For comparison, we show $\sigma_{I}^{2}$ according to the approximation [33, Eq. (31)] along with the exact result (obtained from Monte Carlo simulation) for an i.i.d. Rayleigh fading channel with the same number of transmit and receive antennas. It is clearly seen that both types of channels exhibit similar MI variance behavior as a function of $M_{\mathrm{T}}$, and that $\sigma_{\hat{I}}^{2} \approx 2 \sigma_{I}^{2}$.

\section{MEASUREMENTS}

In this section, we provide results from measurements taken with a commercially employed TDMS-based MIMO channel sounder by applying the rank-1 "calibration procedure" discussed in the beginning of Section $\nabla \mathrm{VI}$. Before elaborating on the measurement results and comparing them with our analytic results, we shall verify assumptions on the system model and on the phase error characteristics that were made throughout the paper.

\section{A. Description of the Measurement Setup}

In the following, a MIMO channel snapshot indexed by the superscript ${ }^{(l)}$ with $l=1,2, \ldots, L$ consists of one snapshot of each of the $M_{\mathrm{T}} M_{\mathrm{R}}$ scalar subchannels. Since the physical channel 
is static (i.e., the cable transfer function is static), time-alignment, as discussed in Section $\amalg-C$, is not needed. The scalar subchannel snapshots in the lth MIMO snapshot are taken at times $t_{k}^{(l)}$ $\left(k=1,2, \ldots, M_{\mathrm{T}} M_{\mathrm{R}}\right)$. All measurement results in this section are based on $L=1100$ MIMO snapshots.

Parameters specifying the channel sounder employed and the measurement setup are provided in Table प. The sounder is based on circular convolution and uses a regular sounding pattern according to (26), implying that frequency offset has no impact on MI. As a consequence of (26), the SISO snapshot distance is given by $T_{\mathrm{R}}$. The sounder inserts a "dummy" receive antenna (i.e., one unused SISO snapshot) to accomodate switching between transmit antennas, so that $T_{\mathrm{T}}=\left(M_{\mathrm{R}}+1\right) T_{\mathrm{R}}$. Note that (26) implies that the SISO snapshot corresponding to the dummy receive antenna is simply omitted. The sounding sequence results from a periodically extended m-sequence of length $N$ (chips) with period $N T$, where $1 / T$ is the chip rate.

The phase noise process, in general, has a distinctive low-pass characteristic. Increasing the SISO snapshot time distances, and thereby reducing the rate at which the continuous-time phase noise process is effectively sampled, results in stronger decorrelation of the time-discrete phase noise process underlying the effective MIMO channel. This, in turn, leads to increased error in estimating the MI. On the other hand, the minimum SISO snapshot time distance is determined by the antenna switching speed, the duty cycle (as explained below) and, in particular, the sounding sequence length. Specifically, reducing the sounding sequence length leads to a degradation of the sequence-correlation properties and hence a reduction in sequence SNR, which in turn implies lower measurement SNR. In summary, there is a tradeoff between the sequence SNR, determined by the time-discrete sounding sequence, in particular by its length, and the MI estimation error due to decorrelation of the phase noise process underlying the effective MIMO channel. To further understand this tradeoff, we performed measurements based on two sounder setups, as defined in Table I, with different sequence lengths.

We would like to point out that the various sounder settings (including sounding sequence 
length) can usually not be chosen independently due to hardware limitations of the sounder. One such typical limitation is the overall duty cycle, i.e., the ratio $\eta=M_{\mathrm{T}} M_{\mathrm{R}} N T / T_{\mathrm{MIMO}}$ of sounding time (where the received signal is recorded and processed) to total measurement time, where $T_{\mathrm{MIMO}}$ is the MIMO snapshot distance. Channel sounders typically employ a small overall duty-cycle to limit the real-time signal processing and data-storage requirements. The overall duty-cycle can be separated into one within and one between MIMO snapshot periods given by

$\eta_{\text {intra }}=M_{\mathrm{T}} M_{\mathrm{R}} N T /\left(t_{M_{\mathrm{T}} M_{\mathrm{R}}}^{(l)}-t_{1}^{(l)}+N T\right)$ and $\eta_{\text {inter }}=\left(t_{M_{\mathrm{T}} M_{\mathrm{R}}}^{(l)}-t_{1}^{(l)}+N T\right) / T_{\mathrm{MIMO}}$, respectively, with $\eta=\eta_{\text {intra }} \eta_{\text {inter. }}$ In general, $\eta_{\text {inter }}$ has no influence on the estimated MI as the correlation properties of the channel and/or phase noise across MIMO snapshot periods do not play a role in our considerations. If $\eta$ is fixed, it is therefore preferable to have $\eta_{\text {inter }}$ small and $\eta_{\text {intra }}$ large. In the two setups considered here, this is indeed the case, with $\eta_{\text {inter }}=0.1431$ and $\eta_{\text {intra }}=0.4697$ for the length-511 sequence, and $\eta_{\text {inter }}=0.05$ and $\eta_{\text {intra }}=0.3193$ for the length-31 sequence.

The transfer function of the cable used to connect transmitter and receiver is flat (recall that we are performing the rank-1 calibration procedure decribed in Section VI over the frequency range of interest. In the sounder under consideration, the overall (i.e., effective) channel induced by $s(t)$ and $r(t)$ along with the cable exhibits, however, some delay spread. This is mainly due to oversampling of the signal at the output of the receive frontend filter by a factor of 2 (relative to the chip rate). We estimated the power-delay profile (PDP) of the resulting effective channel and identified the position of its peak. For further processing we used only the signal corresponding to the peak of the effective channel's PDP.

\section{B. Verifying Assumptions on Phase Error Characteristics and System Model}

The purpose of this section is to investigate the general phase error characteristics of the sounder under consideration, to verify our assumptions on the phase error statistics stated in Section II-B2, and to verify the system model assumptions stated in Section II-B3, Correspondingly, the following discussion is organized into three parts. 
1) General phase error characteristics: We start by giving an impression of the phase variation characteristics over different time horizons. Fig. 9[(a) shows the long-term behavior (multiple seconds) of the phase-unwrapped raw channel estimates 14 Each physical SISO channel measured corresponds to the cable connecting transmitter and receiver. Since the cable exhibits a frequencyflat and constant (over time) transfer function, any variation (over time) in the measured channel must necessarily come from channel sounder nonidealities, or more specifically from phase noise and frequency offset in the transmit and receive LOs. It is clearly seen that the unwrapped phase contains a linear component resulting from the carrier frequency offset between transmitter and receiver. Fig. 9 (b) shows the trace of the residual phase obtained by removing the linear component from the overall observed phase. This linear component was estimated 15 to correspond to a relative (w.r.t. the carrier frequency of $5.25 \mathrm{GHz}$ ) frequency offset of $7.2 \cdot 10^{-11}$, indicating excellent performance in terms of carrier frequency accuracy. Moreover, as already pointed out, since we are employing a regular sounding pattern according to (26), a carrier frequency offset does not have impact on MI. Taking a closer look at the phase residual in Fig. 9[(b), we can see that it exhibits two different constituents: a constant "thickness" corresponding to essentially uncorrelated (on the time scale used) random fluctuations and a component of comparatively larger amplitude and higher temporal correlation containing at times abrupt changes (see, e.g., the area marked in Fig. 9(b) $)$. These abrupt phase changes may be caused by spontaneous phase jumps of the reference oscillators, smoothed out by the PLL. If the PLL bandwidth is small compared to the MIMO measurement rate (i.e., the rate at which MIMO snapshots are taken), we can, however, neglect the impact of the abrupt phase changes.

Fig. 10 shows the phase noise trace (i.e., the overall phase after removal of the estimated linear

\footnotetext{
${ }^{14}$ In Figs. 9 and 10, the time axis corresponds to the sequence of scalar subchannel measurements, with a total of $M_{\mathrm{T}} M_{\mathrm{R}} L$ (with $L=1100$ ), taken according to the regular sounding pattern (26).

${ }^{15}$ Note that the low duty-cycle between MIMO snapshots increases the possibility of the phase containing jumps larger than $2 \pi$ between consecutive MIMO snapshots, thereby resulting in erroneous unwrapping and hence carrier frequency offset estimation errors. Estimation was performed through least-squares fitting.
} 
component) during one MIMO snapshot period taken with 16 length-31 and length-511 sounding sequences, respectively. Each dot in the two figures represents the phase of one scalar subchannel measurement (note the different absolute time scales in Figs. 10[a) and 10[(b)]. In accordance with what was said earlier, one can immediately see that the phase trace corresponding to the length-31 sequence shows significantly higher correlation between successive SISO snapshots than that for the length-511 sequence (which can essentially be considered i.i.d.). While the shorter (length-31) sequence thus is clearly preferable regarding the phase noise properties, it may fail to yield sufficiently high sequence and consequently measurement SNR (see the discussion in Section VII-A). The corresponding estimated rms standard deviation $\hat{\sigma}_{\varphi}$ (estimated by averaging the standard deviation per MIMO snapshot over all $L=1100$ MIMO snapshots) was found to be $3.9^{\circ}$ for the length-31 sequence and $3.8^{\circ}$ for the length-511 sequence. These values agree very well with the $3.5^{\circ} \mathrm{rms}$ value used as "typical" case throughout the paper.

2) Verification of assumptions on phase error statistics: We start by showing, in Fig. 11, the cdf of the measured MIMO snapshot phase noise traces (i.e., the overall phase after removal of the estimated linear component), normalized to zero-mean, and plotted on a scale where Gaussian distributions show a linear behavior. The normalization of the mean was performed by computing and subtracting the empirical mean on a MIMO snapshot by MIMO snapshot basis. It is clearly seen that both for the length-31 and the length-511 sounding sequence an excellent match with an $\mathcal{N}\left(0,\left(3.9^{\circ}\right)^{2}\right)$ distribution is obtained. This allows us to conclude that the assumption of a Gaussian phase noise process (see Section $\llbracket-B 2$ ) is well justified.

The main differentiating factor between the phase noise models discussed in Section $\amalg-B 2$ is stationarity. It therefore remains to verify the stationarity assumption (on the time-scale of one MIMO snapshot) made throughout the paper. In particular, we need to show that the phase noise sequence $\varphi_{n}$ resulting from samples taken at the time instants $n T_{R}$ exhibits stationary

\footnotetext{
${ }^{16}$ Note that the length of the sounding sequences is in fact $31 T$ and $511 T$, respectively.
} 
behavior. This can be done by examining the phase differences $\alpha(k, n)=\varphi_{k}-\varphi_{k+n}$, or more specifically the variance $\sigma_{\alpha}^{2}(k, n)=\mathbb{E}\left\{\alpha^{2}(k, n)\right\}$ (note that $\mathbb{E}\{\alpha(k, n)\}=0$ ). In the case of stationary phase noise, we have $\sigma_{\alpha}^{2}(k, n)=\sigma_{\alpha}^{2}(n)=2 \sigma_{\varphi}^{2}\left(1-r_{\varphi \varphi}(n)\right)$, where $r_{\varphi \varphi}(n)$ denotes the phase noise autocorrelation function. Assuming that the stationary process has limited memory, i.e., $\left|r_{\varphi \varphi}(n)\right| \rightarrow 0$ for $|n| \rightarrow \infty$, we have $\lim _{n \rightarrow \infty} \sigma_{\alpha}^{2}(n)=2 \sigma_{\varphi}^{2}$. On the other hand, if the nonstationary phase noise model, described in Section II-B2, would be applicable, we would have $\sigma_{\alpha}^{2}(k, n)=\sigma_{\alpha}^{2}(n) \propto|n|[23$, Lemma 8.2].

Fig. 12 shows the estimate $\sqrt{\hat{\sigma}_{\alpha}^{2}(n) / 2}$ for the length-31 and the length-511 sounding sequence, where $\hat{\sigma}_{\alpha}^{2}(n)$ is obtained as follows. The measured phase noise sequence $\varphi_{k}$ corresponds to the time instants $t_{k}$. Consequently, $\varphi_{k}$ is the result of sampling at a rate of $1 / T_{R}$, omitting, as already pointed out, the samples corresponding to the dummy receive antenna. Finally, $\mathbb{E}\left\{\alpha^{2}(k, n)\right\}$ is estimated by averaging $\alpha^{2}(k, n)$ in each MIMO snapshot, over all indices $k$ and $n$ satisfying $t_{k+n}-t_{k}=n T_{R}$ with $k \in\left[1, M_{\mathrm{T}} M_{\mathrm{R}}\right]$ and $k+n \in\left[1, M_{\mathrm{T}} M_{\mathrm{R}}\right]$, and then averaging the results over all snapshot periods $l \in[1, L]$. We can clearly see that, for both sampling sequence lengths, $\sqrt{\hat{\sigma}_{\alpha}^{2}(n) / 2}$ levels out at a constant value, which allows us to conclude that over the time frame of interest (i.e., one MIMO snapshot period) the assumption of stationary phase noise is well justified. Moreover, we can observe that the large- $n$ limit of $\sqrt{\hat{\sigma}_{\alpha}^{2}(n) / 2}$ is close to $4^{\circ}$, which is very well in accordance with the $3.8^{\circ}$ and $3.9^{\circ} \mathrm{rms}$ values estimated earlier.

3) Verification of the system model assumptions: Based on our measurement results, we shall next quantify the impact of phase errors on sequence SNR and discuss the validity of the assumptions (18) and (19).

While we have seen in Section $\amalg$ I-B3 that it is safe to assume the absence of peak-shifting in the sounder under consideration (recall that the sounder employs m-sequences), phase errors will, in general, lead to a reduction in sequence SNR and hence also in measurement SNR. 
Defining

$$
\mathrm{SNR}_{i}=\frac{\left|c^{\prime}(0)\right|^{2}}{\left|c^{\prime}(i T)\right|^{2}}, \quad i=1,2, \ldots, N-2
$$

we estimate (Monte Carlo) the histogram of the overall SNR, defined through its histogram to be given by the average of the histograms corresponding to the quantities $\mathrm{SNR}_{i} ; 10000$ phase noise realizations per lag were used. In the absence of phase noise an m-sequence has a (deterministic) sequence SNR of $20 \log _{10}(m)$, which, for $m=511$, equals $54.2 \mathrm{~dB}$. The phase noise in the sounder under consideration randomizes this value to a mean of $53.8 \mathrm{~dB}$ and a level below $49.1 \mathrm{~dB}$ w.p. $0.1 \%$. We will see later that $49.1 \mathrm{~dB}$ lies above the sounder's SNR caused by thermal noise. Consequently, the effect of phase noise on sequence SNR, albeit noticeable, can be considered insignificant.

Condition (18) is verified as follows. We start by noting that, for a periodically extended msequence and circular convolution in the receiver, as employed in the sounder under consideration, the integration interval in (18) has length $\left|\mathfrak{I}_{\mathrm{P}}\right|=N T$. With $\Delta \omega=2 \pi \Delta f=2.4 \mathrm{rad} / \mathrm{s}, N T=$ $310 \mathrm{~ns}$ for the length-31 sounding sequence, and $N T=5110 \mathrm{~ns}$ for the length-511 sounding sequence, we have $|\Delta \omega \xi| \leq 12.3 \cdot 10^{-6} \mathrm{rad}$, which implies that we can safely assume that $\exp (j \Delta \omega \xi) \approx 1$. If the inverse of the bandwidth of $\varphi_{\mathrm{R}}(t)$ and $\varphi_{\mathrm{T}}(t)$ is much larger than $N T$ and/or the variance of $\varphi_{\mathrm{R}}(t)$ and $\varphi_{\mathrm{T}}(t)$ is small, the term $\exp \left(-j\left[\varphi_{\mathrm{R}}\left(t_{k}+\xi\right)-\varphi_{\mathrm{T}}\left(t_{k}-\right.\right.\right.$ $\left.\left.\tau_{k}^{(l)}+\xi\right)\right]$ ) will remain essentially constant over an interval of length $N T$ thereby, together with $\exp (j \Delta \omega \xi) \approx 1$, resulting in

$$
\left|\int_{\mathfrak{I}_{\mathrm{P}}} e^{j \Delta \omega \xi} e^{-j \varphi_{\mathrm{R}}\left(t_{k}+\xi\right)} e^{j \varphi_{\mathrm{T}}\left(t_{k}-\tau_{k}^{(l)}+\xi\right)} \tilde{w}\left(\xi-\tau_{k}^{(l)}\right) \mathrm{d} \xi\right| \approx\left|\int_{\mathfrak{I}_{\mathrm{P}}} \tilde{w}\left(\xi-\tau_{k}^{(l)}\right) \mathrm{d} \xi\right|=1
$$

which confirms (18). From Fig. 12, we can see that the phase noise process' bandwidth is small enough for phase noise to sustain a correlation coefficient of at least 0.75 over the duration of a length-511 sounding sequence. In addition the phase noise variance is very small as well. 
Condition (19) requires that the inverse of the coherence bandwidth of the phase noise process $\varphi_{\mathrm{T}}(t)$ is large compared to the maximum multipath delay difference. From Fig. 12, we can conclude that for the sounder under consideration this is the case only for multipath delay differences of up to $5 \mu$ s, i.e., only for indoor or small-cell cellular outdoor propagation scenarios. Consequently, assumption (19) needs to be carefully verified on a case by case basis for outdoor measurments.

Finally, even though only relevant in the case of linear convolution-based sounders, we would like to comment on the condition $\Delta \omega \tau_{k}^{(l)} \approx 0, \forall k, l$. We start by noting that typical values for maximum multipath delay differences range from $1 \mu$ s for indoor environments, over $10 \mu$ s for outdoor small-cell cellular systems (with a few kilometers cell size), to $100 \mu$ s for a propagation range of $30 \mathrm{~km}$ encountered in outdoor large-cell cellular systems. With $\Delta \omega=2.4 \mathrm{rad} / \mathrm{s}$, this corresponds to $\Delta \omega \tau_{k}^{(l)}$ ranging from $2.4 \cdot 10^{-6} \mathrm{rad}$ to $2.4 \cdot 10^{-4} \mathrm{rad}$. We can therefore conclude that the condition $\Delta \omega \tau_{k}^{(l)} \approx 0$ is well satisfied in practice.

Other imperfections: Finally, before quantifying the impact of phase errors on estimated MI in our calibration measurement, we need to convince ourselves that the errors observed are, indeed, phase errors and are not caused by other measurement imperfections. This can be done as follows. We start by noting that, in polar coordinates, phase errors are visible only in the argument of the effective channel and not in the magnitude. Assuming that all additional error sources can be subsumed as additive noise $n$ which is circularly symmetric distributed, we can measure the SNR in the magnitude direction which, due to

$$
\left|h e^{j \varphi}+n\right|^{2} \stackrel{\mathrm{d}}{=}\left|h e^{j \varphi}+n e^{j \varphi}\right|^{2} \stackrel{\mathrm{d}}{=}|h+n|^{2}
$$

is simply the SNR of $h+n$. By circular symmetry of the additive noise term, the result of this measurement provides us with the noise caused by other error sources in the direction orthogonal to the magnitude direction which, by the small phase noise assumption is the phase direction. 
This leads to an SNR level (in phase direction) of below $37.5 \mathrm{~dB}$ w.p. $1 \%$ for the length-511 sequence. Compared to the corresponding phase noise SNR level of below $15.1 \mathrm{~dB}$ w.p. $1 \%$, we can conclude that phase noise is, indeed, the dominating error source.

\section{Estimated MI for a Rank-1 Physical Channel}

Next, we illustrate the impact of phase errors on estimated MI by performing the rank-1 "calibration procedure" described in the first paragraph of Section VI. For brevity, we restrict ourselves to the length-511 sounding sequence (where phase noise is essentially fully uncorrelated).

For an SNR of $\rho=30 \mathrm{~dB}$, Fig. 13(a) shows the MI obtained from the measurements along with the corresponding $\mathrm{MI}$ of the rank-1 physical channel $\mathbf{H}=\mathbf{1}$. To illustrate the impact of additive noise on the channel coefficients, we also show the capacity of a rank-1 physical channel subject to additive white Gaussian noise (AWGN) on the channel coefficients, i.e., $\operatorname{vec}(\widehat{\mathbf{H}}) \stackrel{\mathrm{d}}{=} \mathcal{C N}\left(\alpha \mathbf{1}, \sigma_{N}^{2} \mathbf{I}\right)$ where $\alpha$, real-valued and positive, and $\sigma_{N}^{2}$ were estimated (using the moment-matching method [56]) from the measurement results 17 on a MIMO snapshot by MIMO snapshot basis. The corresponding per-subchannel SNR is hence given by $\mathrm{SNR}=\alpha^{2} / \sigma_{N}^{2}$. While additive errors on the channel coefficients do not impact the capacity significantly, we can see that the MI of the effective channel is more than $200 \%$ higher than the MI of the underlying physical channel. This significant measurement error can alternatively be quantified in terms of the MIMO channel's number of spatial degrees of freedom, using the definition provided in [8] as

$$
m_{\text {free }}=I_{\rho}-I_{\rho / 2}
$$

Fig. 13(b) shows $m_{\text {free }}$ corresponding to Fig. 13(a), We can see that the presence of phase noise increases $m_{\text {free }}$ from 1 for the rank-1 physical channel to 8-9 for the measured channel.

\footnotetext{
${ }^{17}$ The entries of the effective MIMO channel are, in general, not unit-modulus (due to, e.g., additive thermal noise).
} 
An effective full-rank channel, i.e., a channel where all eigenvalues are significant at the given SNR, would correspond to $m_{\text {free }}=16$. Moreover, in Fig. 14 we show the ergodic capacity corresponding to the measurement results in Fig. 13, i.e., averaged over $L=1100 \mathrm{MIMO}$ snapshots at each SNR, along with the ergodic capacity predicted by our analytic results in Theorems 7 and 9 for $3.8^{\circ} \mathrm{rms}$ fully uncorrelated phase noise. We can see that the analytic lower and upper bounds are slightly higher than the measured ergodic capacity. This is probably due to the residual (very low since we are using length-511 sounding sequences) correlation in the phase noise process, neglected in the analytic results. Apart from this effect, the measurement results exhibit an excellent match with the analytic results.

\section{CONCLUSIONS}

We showed that phase errors (caused by phase noise and carrier frequency offset) in timedivision multiplexed switching (TDMS)-based multiple-input multiple-output (MIMO) radio channel sounders can alter the channel statistics significantly and thereby lead to severe mutual information (MI) and capacity estimation errors. The impact of phase errors is most pronounced for low-rank physical channels where overestimation by several hundred percent can occur. A detailed analysis of the rank-1 physical MIMO channel revealed that realistic phase noise properties lead to a decorrelation of the channel matrix and result in a full-rank effective channel. Our analytic results are supported by measurements conducted with a commercially employed TDMS-based MIMO channel sounder. In the particular measurement setup considered, phase noise turned a physical rank-1 channel into an effective rank-8 channel. In general, for a given sounder, the impact of phase errors will be more pronounced for a larger number of antennas and for larger SISO snapshot time distances. Our analysis furthermore demonstrated that the MI measurement error induced by phase errors can depend significantly on the order in which the individual subchannels of the overall MIMO channel matrix are measured.

Based on matrix differential calculus and matrix-variate Wirtinger calculus, we characterized 
the sensitivity of MI w.r.t. phase errors.

The presence of a carrier frequency offset between transmitter and receiver was found to be less problematic as it does not impact the MI of the physical channel under quite general conditions on the sounding pattern. Moreover, the impact of a carrier frequency offset is of deterministic nature and can be compensated by estimating the offset and subsequently removing it.

In the light of the main findings of this paper, the results obtained through MIMO channel measurement campaigns using TDMS-based MIMO channel sounders should be interpreted with great care. To the best of our knowledge, the large majority of commercially available MIMO channel sounders is TDMS-based. In particular, measurement results reporting the absence of pin-hole or key-hole channels [2], [3], seem questionable unless a channel sounder with separate radio frequency $(\mathrm{RF})$ chains for the individual transmit and/or receive antenna elements is used.

Simple averaging (w.r.t. phase noise realizations) of MI is not a viable way to mitigate the effect of phase errors as MI is a nonlinear function of the phase noise samples and of the physical channel realization. Averaging of entries of the effective channel matrix over different MIMO snapshots is not a viable option either as it leads to a significant reduction in measurement SNR; this is due to the phase offsets between MIMO snapshots essentially being arbitrary. While this problem could, in principle, be mitigated through an increased MIMO measurement rate, the latter is often limited by requirements on the minimum sequence length, maximum duty cycle, and minimum switching times.

A mitigation method based on taking multiple measurements per scalar subchannel at a higher rate with the goal of improving the phase stability across antennas and, ideally, emulating onesided switching, is described, along with the corresponding tradeoffs, in [57]. This method yields excellent results in the case of $\operatorname{small} \min \left(M_{\mathrm{T}}, M_{\mathrm{R}}\right)$.

The safest, but most expensive, solution to avoid measurement errors due to phase errors is to employ a channel sounder with separate RF chains for the individual transmit and/or receive antenna elements. We hasten to add, however, that such an architecture has other problems 
associated with it, such as gain/phase imbalances and crosstalk between the different RF chains. Nevertheless, at least in theory, as shown in Section IV-A3, if either the transmit antenna array or the receive antenna array employs a separate RF chain for each antenna element and TDMS is used on the other side of the link, MI is not affected by phase errors. If such a sounder architecture cannot be realized, the local oscillators (LOs) should be selected very carefully or even custom-designed which can be very expensive. As a last resort, we recommend to synchronize the LOs at transmitter and receiver through a cable. This solution, however, makes outdoor measurements difficult and limits the flexibility of the sounding device, in particular w.r.t. the distance between transmitter and receiver.

\section{APPENDIX A}

Theorem 10 (Fang, Kotz, and $N g$ [14, Th. 1.4]): Let $\mathbf{x} \stackrel{\mathrm{d}}{=} D_{n}(\mathbf{a})$ be partitioned into $k$ subvectors $\mathbf{x}^{(1)}, \mathbf{x}^{(2)}, \ldots, \mathbf{x}^{(k)}$ and $\mathbf{a}$ into the corresponding subvectors $\mathbf{a}^{(1)}, \mathbf{a}^{(2)}, \ldots, \mathbf{a}^{(k)}$. Let $y_{i}$ and $b_{i}$ be, respectively, the sums of the components of $\mathbf{x}^{(i)}$ and $\mathbf{a}^{(i)}$, and set $\mathbf{z}^{(i)}=\mathbf{x}^{(i)} / y_{i}$. The following statements hold:

i) The vectors $\mathbf{z}^{(i)}, \mathbf{z}^{(i+1)}, \ldots, \mathbf{z}^{(k)}, \mathbf{y}=\left[\begin{array}{llll}y_{1} & y_{2} & \cdots & y_{k}\end{array}\right]^{T}$ are statistically independent.

ii) $\mathbf{y}$ is distributed as $D_{k}\left(b_{1}, b_{2}, \ldots, b_{k}\right)$.

iii) $\mathbf{z}^{(i)}$ is distributed as $D_{n_{i}}\left(\mathbf{a}^{(i)}\right), i=1,2, \ldots, k$, where $n_{i}$ is the number of elements in the subvectors $\mathbf{a}^{(i)}$ and $\mathbf{z}^{(i)}$.

From Theorem 10 it follows immediately that the vectors $\mathbf{x}^{(i)}$ are Dirichlet (or beta) distributed. This result can be stated formally as follows.

Theorem 11 (Fang, Kotz, and $\mathrm{Ng}$ [14, Th. 1.5]): If $\left[\begin{array}{llll}x_{1} & x_{2} & \cdots & x_{n-1}\end{array}\right] \stackrel{\mathrm{d}}{=} D_{n-1}\left(a_{1}, a_{2}, \ldots\right.$, $\left.a_{n-1} ; a_{n}\right)$, then for any $k<n-1$, we have $\left[\begin{array}{llll}x_{1} & x_{2} & \cdots & x_{k}\end{array}\right] \stackrel{\mathrm{d}}{=} D_{k}\left(a_{1}, a_{2}, \ldots, a_{k} ; b\right)$, where $b=a_{k+1}+a_{k+2}+\cdots+a_{n}$. In particular, for any $i(i=1,2, \ldots, n-1)$, we have $x_{i} \stackrel{\mathrm{d}}{=} \beta\left(a_{i}, a-a_{i}\right)$ with $a=\sum_{i=1}^{n} a_{i}$. 
Lemma 4: The first derivative $\Psi^{\prime}(z)=\mathrm{d} \Psi(z) / \mathrm{d} z$ of the digamma function $\Psi(z)$ defined in (2) satisfies

$$
\Psi^{\prime}(z) \leq \frac{1}{2} \Psi^{\prime}\left(\frac{z}{2}\right)
$$

Proof: Using (4), we have

$$
\begin{aligned}
\Psi^{\prime}\left(\frac{x}{2}\right) & =\sum_{p=0}^{\infty} \frac{1}{\left(p+\frac{x}{2}\right)^{2}}=\sum_{p=0}^{\infty} \frac{4}{(2 p+x)^{2}}=4\left(\frac{1}{x^{2}}+\frac{1}{(2+x)^{2}}+\frac{1}{(4+x)^{2}}+\cdots\right) \\
& =4\left(\sum_{p=0}^{\infty} \frac{1}{(p+x)^{2}}-\sum_{p=0}^{\infty} \frac{1}{((2 p+1)+x)^{2}}\right)=4 \Psi^{\prime}(x)-\Psi^{\prime}\left(\frac{x+1}{2}\right)
\end{aligned}
$$

which upon noting that

$$
\Psi^{\prime}\left(\frac{x}{2}\right) \geq \Psi^{\prime}\left(\frac{x+1}{2}\right)
$$

completes the proof.

Lemma 5: The eigenvalues of the matrix

$$
\operatorname{Re}\left(\mathbf{A}_{i}\right)=\mathbf{I}_{M_{\mathrm{T}}}-\sum_{n=1}^{i-1} \frac{\operatorname{Re}\left(\mathbf{s}_{n}^{\perp} \mathbf{s}_{n}^{\perp H}\right)}{\left\|\mathbf{s}_{n}^{\perp}\right\|^{2}}, \quad i=2,3, \ldots, M_{\mathrm{R}}
$$

with $\mathbf{A}_{i}$ and $\mathbf{s}_{n}^{\perp}$ defined in (53) and $\mathbf{s}_{n}$ defined through $\mathbf{S}=\left[\begin{array}{llll}\mathbf{s}_{1} & \mathbf{s}_{2} & \ldots & \mathbf{s}_{M_{\mathrm{R}}}\end{array}\right]^{T}=-j \boldsymbol{\Sigma}+\widetilde{\boldsymbol{\Phi}}$ with $\Sigma$ as in (51), are given by $\left(k=1,2, \ldots, M_{\mathrm{T}}\right)$

$$
\left\{\sigma_{k}^{(i)}\right\}=\{\underbrace{1, \ldots, 1}_{M_{\mathrm{T}}-i}, \underbrace{0, \ldots, 0}_{i-2}, \eta^{(i)}, 1-\eta^{(i)}\}
$$

where $\eta^{(i)}=\eta^{(i)}\left(\mathbf{s}_{1}^{\perp}, \mathbf{s}_{2}^{\perp}, \ldots, \mathbf{s}_{i-1}^{\perp}\right) \in[0,1]$.

Proof: We start by writing

$$
\begin{aligned}
\operatorname{Re}\left(\mathbf{A}_{i}\right) & =\mathbf{I}_{M_{\mathrm{T}}}-\sum_{n=1}^{i-1} \frac{\operatorname{Re}\left(\mathbf{s}_{n}^{\perp}\right)\left(\operatorname{Re}\left(\mathbf{s}_{n}^{\perp}\right)\right)^{T}}{\left\|\mathbf{s}_{n}^{\perp}\right\|^{2}}-\sum_{n=1}^{i-1} \frac{\operatorname{Im}\left(\mathbf{s}_{n}^{\perp}\right)\left(\operatorname{Im}\left(\mathbf{s}_{n}^{\perp}\right)\right)^{T}}{\left\|\mathbf{s}_{n}^{\perp}\right\|^{2}} \\
& =\mathbf{I}_{M_{\mathrm{T}}}-\mathbf{G}_{i} \mathbf{G}_{i}^{T}
\end{aligned}
$$


where

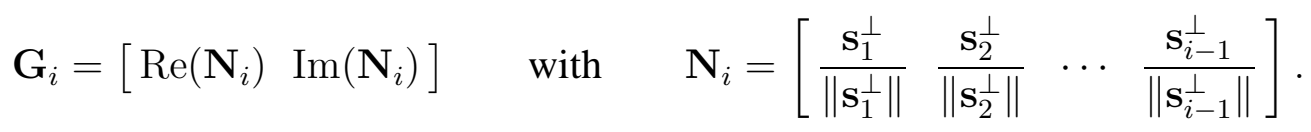

By slight abuse of notation, in the remainder of this proof, we let $\lambda_{k}(\mathbf{X})$ denote the unordered eigenvalues of the matrix $\mathbf{X}$. It follows that $\sigma_{k}^{(i)}=1-\lambda_{k}\left(\mathbf{G}_{i} \mathbf{G}_{i}^{T}\right), k=1,2, \ldots, M_{\mathrm{T}}$. Invoking Lemma 6, we can conclude that the $2(i-1)$ eigenvalues $\lambda_{k}\left(\mathbf{G}_{i}^{T} \mathbf{G}_{i}\right)$ are given by

$$
\left\{\frac{1}{2}+\frac{1}{2} \sqrt{\mu_{1}^{(i)}}, \frac{1}{2}-\frac{1}{2} \sqrt{\mu_{1}^{(i)}}, \ldots, \frac{1}{2}+\frac{1}{2} \sqrt{\mu_{i-1}^{(i)}}, \frac{1}{2}-\frac{1}{2} \sqrt{\mu_{i-1}^{(i)}}\right\}
$$

with $\mu_{k}^{(i)} \in \mathbb{R}(k=1,2, \ldots, i-1)$. Therefore, when paired properly, the $\lambda_{k}\left(\mathbf{G}_{i}^{T} \mathbf{G}_{i}\right)$ pairwise add up to 1 . This property will next allow us to show that $\mathbf{G}_{i}^{T} \mathbf{G}_{i}$ has $i-2$ eigenvalues equal to $1, i-2$ eigenvalues equal to 0 , and one pair of eigenvalues given by $\left\{\eta^{(i)}, 1-\eta^{(i)}\right\}$. We start by noting that, using (53), it follows for $i=2,3, \ldots, M_{\mathrm{R}}$ that (recall that $\mathbf{s}_{i} \in \mathbb{R}^{M_{\mathrm{T}}}$ )

$$
\begin{aligned}
\operatorname{Im}\left(\frac{\mathbf{s}_{i}^{\perp}}{\left\|\mathbf{s}_{i}^{\perp}\right\|}\right) & =-\sum_{n=1}^{i-1} \frac{\operatorname{Im}\left(\mathbf{s}_{n}^{\perp} \mathbf{s}_{n}^{\perp H}\right)}{\left\|\mathbf{s}_{n}^{\perp}\right\|^{2}} \frac{\mathbf{s}_{i}}{\left\|\mathbf{s}_{i}^{\perp}\right\|} \\
& =-\sum_{n=1}^{i-1} \frac{\operatorname{Im}\left(\mathbf{s}_{n}^{\perp}\right)\left(\operatorname{Re}\left(\mathbf{s}_{n}^{\perp}\right)\right)^{T}-\operatorname{Re}\left(\mathbf{s}_{n}^{\perp}\right)\left(\operatorname{Im}\left(\mathbf{s}_{n}^{\perp}\right)\right)^{T}}{\left\|\mathbf{s}_{n}^{\perp}\right\|^{2}} \frac{\mathbf{s}_{i}}{\left\|\mathbf{s}_{i}^{\perp}\right\|} \\
& =\sum_{n=1}^{i-1}\left(\operatorname{Im}\left(\mathbf{s}_{n}^{\perp}\right) \xi_{n}+\operatorname{Re}\left(\mathbf{s}_{n}^{\perp}\right) \zeta_{n}\right) \\
\operatorname{Re}\left(\frac{\mathbf{s}_{i}^{\perp}}{\left\|\mathbf{s}_{i}^{\perp}\right\|}\right) & =\left(\mathbf{I}_{M_{\mathrm{T}}}-\sum_{n=1}^{i-1} \frac{\operatorname{Re}\left(\mathbf{s}_{n}^{\perp}\right)\left(\operatorname{Re}\left(\mathbf{s}_{n}^{\perp}\right)\right)^{T}}{\left\|\mathbf{s}_{n}^{\perp}\right\|^{2}}-\sum_{n=1}^{i-1} \frac{\operatorname{Im}\left(\mathbf{s}_{n}^{\perp}\right)\left(\operatorname{Im}\left(\mathbf{s}_{n}^{\perp}\right)\right)^{T}}{\left\|\mathbf{s}_{n}^{\perp}\right\|^{2}}\right) \frac{\mathbf{s}_{i}}{\left\|\mathbf{s}_{i}^{\perp}\right\|} \\
& =\frac{\mathbf{s}_{i}}{\left\|\mathbf{s}_{i}^{\perp}\right\|}+\sum_{n=1}^{i-1}\left(\operatorname{Re}\left(\mathbf{s}_{n}^{\perp}\right) \xi_{n}+\operatorname{Im}\left(\mathbf{s}_{n}^{\perp}\right) \zeta_{n}\right)
\end{aligned}
$$

with $\xi_{n}, \zeta_{n} \in \mathbb{R}$. The significance of (81) and (82) is that it can be used to show that $\mathrm{r}\left(\mathbf{G}_{i}\right)=$ $i$. More specifically, starting with $i=2$, we note that $\mathbf{G}_{2}=\left[\operatorname{Re}\left(\mathbf{s}_{1} /\left\|\mathbf{s}_{1}\right\|\right) \operatorname{Im}\left(\mathbf{s}_{1} /\left\|\mathbf{s}_{1}\right\|\right)\right]$ has rank 2 w.p.1 as will be shown first. Noting that $\operatorname{Re}\left(\mathbf{s}_{1}\right) \stackrel{\mathrm{d}}{=} \mathcal{N}\left(\mathbf{0}, \sigma_{\varphi}^{2} \mathbf{I}\right)$ and $\operatorname{Im}\left(\mathbf{s}_{1}\right)=$ $\left[\begin{array}{ll}-M_{\mathrm{T}} M_{\mathrm{R}} & \mathbf{0}_{1, M_{\mathrm{T}}-1}\end{array}\right]^{T}$, it follows that $\left\|\mathbf{s}_{1}\right\|>0$ w.p.1 and hence $r\left(\mathbf{G}_{2}\right)=r\left(\left[\operatorname{Re}\left(\mathbf{s}_{1}\right) \operatorname{Im}\left(\mathbf{s}_{1}\right)\right]\right)$. 
By definition, $\operatorname{Re}\left(\mathbf{s}_{1}\right)$ and $\operatorname{Im}\left(\mathbf{s}_{1}\right)$ are linearly independent w.p.1 and hence $r\left(\mathbf{G}_{2}\right)=2$ w.p.1. Now

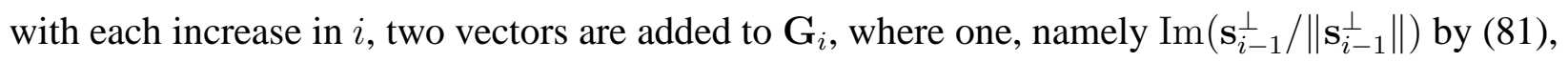
is a linear combination of the vectors already in $\mathbf{G}_{i}$, and the other one, namely $\operatorname{Re}\left(\mathbf{s}_{i-1}^{\perp} /\left\|\mathbf{s}_{i-1}^{\perp}\right\|\right)$ by (82), is a linear combination of the vectors already in $\mathbf{G}_{i}$ and the vector $\mathbf{s}_{i-1} /\left\|\mathbf{s}_{i-1}^{\perp}\right\|$. We can therefore conclude that the $2(i-1) \times 2(i-1)$ matrix $\mathbf{G}_{i}^{T} \mathbf{G}_{i}$ has at most $i$ nonzero eigenvalues. The remaining $i-2$ eigenvalues are equal to zero. By (80) we must therefore have $i-2$ eigenvalues which are equal to 1 . Since we have $2 i-2$ eigenvalues in total, it follows that there is one pair of eigenvalues of the form $\left\{\eta^{(i)}, 1-\eta^{(i)}\right\}$. Finally, noting that $\eta^{(i)}$ and $1-\eta^{(i)}$ must be real-valued and positive, we can conclude that $\eta^{(i)} \in[0,1]$.

Lemma 6: Given an orthonormal set of vectors $\mathbf{x}_{1}, \mathbf{x}_{2}, \ldots, \mathbf{x}_{n} \in \mathbb{C}^{N}$ with $n \leq N$, the eigenvalues of the $2 n \times 2 n$ matrix $\mathbf{Y}^{T} \mathbf{Y}$ with

$$
\mathbf{Y}=\left[\begin{array}{ll}
\operatorname{Re}(\mathbf{X}) & \operatorname{Im}(\mathbf{X})
\end{array}\right]
$$

where

$$
\mathbf{X}=\left[\begin{array}{llll}
\mathbf{x}_{1} & \mathbf{x}_{2} & \cdots & \mathbf{x}_{n}
\end{array}\right]
$$

are given by

$$
\begin{aligned}
\left\{\frac{1+\sqrt{\lambda_{1}(\mathbf{A})}}{2}, \frac{1-\sqrt{\lambda_{1}(\mathbf{A})}}{2}, \frac{1+\sqrt{\lambda_{2}(\mathbf{A})}}{2}, \frac{1-\sqrt{\lambda_{2}(\mathbf{A})}}{2}, \ldots,\right. & \left.\frac{1+\sqrt{\lambda_{n}(\mathbf{A})}}{2}, \frac{1-\sqrt{\lambda_{n}(\mathbf{A})}}{2}\right\}
\end{aligned}
$$

where $\mathbf{A}=\mathbf{X}^{T} \mathbf{X} \mathbf{X}^{H} \mathbf{X}^{*}$.

Proof: We start by noting that

$$
\mathbf{Y}^{\prime}=\sqrt{\frac{1}{2}}\left[\begin{array}{ll}
\mathbf{X} & \mathbf{X}^{*}
\end{array}\right]
$$


satisfies

$$
\mathbf{Y}^{\prime}=\mathbf{Y} \underbrace{\left(\sqrt{\frac{1}{2}}\left[\begin{array}{cc}
1 & 1 \\
j & -j
\end{array}\right] \otimes \mathbf{I}_{n}\right)}_{\mathbf{U}}
$$

where $\mathbf{U}$ is unitary. We can therefore conclude that

$$
\lambda_{i}\left(\mathbf{Y}^{\prime H} \mathbf{Y}^{\prime}\right)=\frac{1}{2} \lambda_{i}\left(\left[\begin{array}{c}
\mathbf{X}^{H} \\
\mathbf{X}^{T}
\end{array}\right]\left[\begin{array}{ll}
\mathbf{X} & \mathbf{X}^{*}
\end{array}\right]\right)=\lambda_{i}\left(\mathbf{U}^{H} \mathbf{Y}^{H} \mathbf{Y} \mathbf{U}\right)=\lambda_{i}\left(\mathbf{Y}^{H} \mathbf{Y}\right)
$$

Next, we note that $\mathbf{X}^{H} \mathbf{X}=\mathbf{X}^{T} \mathbf{X}^{*}=\mathbf{I}_{n}$ due to the orthonormality of the $\mathbf{x}_{n}$, and hence the eigenvalues of $\mathbf{Y}^{H} \mathbf{Y}$ are given by the solutions of the characteristic equation

$$
\mathrm{p}(\mu)=\operatorname{det}\left(\left[\begin{array}{cc}
(1-2 \mu) \mathbf{I} & \mathbf{X}^{H} \mathbf{X}^{*} \\
\mathbf{X}^{T} \mathbf{X} & (1-2 \mu) \mathbf{I}
\end{array}\right]\right)
$$

For a Hermitian matrix $\mathrm{S}$ partitioned according to

$$
\mathbf{S}=\left[\begin{array}{cc}
\mathrm{A} & \mathbf{B} \\
\mathbf{B}^{*} & \mathrm{C}
\end{array}\right]
$$

where $\mathbf{A}$ and $\mathbf{C}$ are square matrices, we have from the Schur complement formula [43, Sec. 0.8.5]

$$
\operatorname{det}(\mathbf{S})=\operatorname{det}(\mathbf{A}) \operatorname{det}\left(\mathbf{C}-\mathbf{B}^{*} \mathbf{A}^{-1} \mathbf{B}\right)
$$

It therefore follows that

$$
p(\mu)=\operatorname{det}\left((1-2 \mu)^{2} \mathbf{I}-\mathbf{X}^{T} \mathbf{X} \mathbf{X}^{H} \mathbf{X}^{*}\right)
$$

The solution of (84) yields the eigenvalues of $\mathbf{Y}^{H} \mathbf{Y}$ as (83), which completes the proof.

Note that if $n \geq N / 2, \mathbf{Y}$ must have $2 n-N$ eigenvalues equal to 0 and therefore by (83) also 
$2 n-N$ eigenvalues equal to 1 so that $(83)$ can be refined as

$$
\begin{aligned}
\left\{\frac{1+\sqrt{\lambda_{1}(\mathbf{A})}}{2}, \frac{1-\sqrt{\lambda_{1}(\mathbf{A})}}{2}, \frac{1+\sqrt{\lambda_{2}(\mathbf{A})}}{2}, \frac{1-\sqrt{\lambda_{2}(\mathbf{A})}}{2}, \ldots,\right. & \frac{1+\sqrt{\lambda_{N-n}(\mathbf{A})}}{2}, \frac{1-\sqrt{\lambda_{N-n}(\mathbf{A})}}{2} \underbrace{1, \ldots, 1}_{2 n-N}, \underbrace{0, \ldots, 0}_{2 n-N}\} .
\end{aligned}
$$

Theorem 12: Given the conditional (on $\eta$ ) $\mathrm{RV}^{18} Z(\eta) \stackrel{\mathrm{d}}{=} \chi_{1, \sigma_{\varphi}^{2} \eta}^{2}+\chi_{1, \sigma_{\varphi}^{2}(1-\eta)}^{2}$, where the two chi-square distributed terms are independent, and a constant $r \in \mathbb{R}^{+}$, we have

$$
\mathbb{E}\{\log (r+Z(0))\} \leq \mathbb{E}\{\log (r+Z(\eta))\}, \quad 0 \leq \eta \leq 1
$$

Proof: We start by noting that $Z(\eta) \stackrel{\mathrm{d}}{=} Z(1-\eta)$ for $0 \leq \eta \leq 1$ so that it suffices to prove (85) for $0 \leq \eta \leq 1 / 2$. We shall also need the properties $Z(0) \stackrel{\mathrm{d}}{=} Z(1) \stackrel{\mathrm{d}}{=} \chi_{1, \sigma_{\varphi}^{2}}^{2}$ and $Z(1 / 2) \stackrel{\mathrm{d}}{=} \chi_{2, \sigma_{\varphi}^{2} / 2}^{2}$. Noting that

$$
\begin{aligned}
\log (r+Z(\eta)) & =\log (r)+\log (1+Z(\eta) / r) \\
& =\log (r)+\log (1+\tilde{Z}(\eta))
\end{aligned}
$$

where $\tilde{Z}(\eta) \stackrel{\mathrm{d}}{=} \chi_{1, \tilde{\sigma}_{\varphi}^{2} \eta}^{2}+\chi_{1, \tilde{\sigma}_{\varphi}^{2}(1-\eta)}^{2}$ and $\tilde{\sigma}_{\varphi}^{2}=\sigma_{\varphi}^{2} / r$, it follows that proving (85) for $r=1$ is sufficient.

The cdf of $Y(\xi)=\chi_{1, \xi}^{2}$ is [15, Eq. 26.4.19, Eq. 6.5.16, Eq. 6.1.8]

$$
\mathrm{F}_{Y(\xi)}(y)=\operatorname{erf}\left(\sqrt{\frac{y}{2 \xi}}\right)
$$

where $\operatorname{erf}(x)=(2 / \sqrt{\pi}) \int_{0}^{x} \exp \left(-t^{2}\right) \mathrm{d} t$ denotes the error function. The inverse function corresponding to (86) is given by $\mathrm{F}_{Y(\xi)}^{-1}(x)=2 \xi \operatorname{erfi}^{2}(x)$, where erfi $(x)$ denotes the inverse error function. Using the inverse method of generating random deviates [15, Sec. 26.8], i.e., for a

\footnotetext{
${ }^{18}$ For the sake of simplicity of notation, we committed an abuse of notation here, as in the main body (cf. Theorem 5) we used the symbol $Z(\eta)$ to denote the unconditional $\operatorname{RV} Z(\eta)=\sigma_{\varphi}^{2}\left(\eta X_{1}+(1-\eta) X_{2}\right)$.
} 
uniformly distributed RV $U \in[0,1]$ we have $\chi_{1, \xi}^{2} \stackrel{\mathrm{d}}{=} \mathrm{F}_{Y(\xi)}^{-1}(U)$, together with the independence of the two terms in $Z(\eta) \stackrel{\mathrm{d}}{=} \chi_{1, \sigma_{\varphi}^{2} \eta}^{2}+\chi_{1, \sigma_{\varphi}^{2}(1-\eta)}^{2}$, we can now express $Z(\eta)$ in terms of two independent uniformly distributed RVs $U_{1} \in[0,1]$ and $U_{2} \in[0,1]$ as

$$
Z(\eta) \stackrel{\mathrm{d}}{=} 2 \sigma^{2} \eta \operatorname{erfi}^{2}\left(U_{1}\right)+2 \sigma^{2}(1-\eta) \operatorname{erfi}^{2}\left(U_{2}\right)
$$

To prove the Theorem, it suffices to show that

$$
\frac{\partial}{\partial \eta} \mathbb{E}\{\log (1+Z(\eta))\} \geq 0, \quad 0 \leq \eta \leq 1 / 2
$$

Inserting (87) in (88), we get

$$
\begin{aligned}
\frac{\partial}{\partial \eta} \mathbb{E}\{\log (1+Z(\eta))\} & =\frac{\partial}{\partial \eta} \int_{0}^{1} \int_{0}^{1} \log \left(1+2 \sigma^{2} \eta \operatorname{erfi}^{2}\left(u_{1}\right)+2 \sigma^{2}(1-\eta) \operatorname{erfi}^{2}\left(u_{2}\right)\right) \mathrm{d} u_{1} \mathrm{~d} u_{2} \\
& \stackrel{(a)}{=} \log (e) \int_{0}^{1} \int_{0}^{1} \frac{2 \sigma^{2}\left(\operatorname{erfi}^{2}\left(u_{1}\right)-\operatorname{erfi}^{2}\left(u_{2}\right)\right)}{1+2 \sigma^{2} \eta \operatorname{erfi}^{2}\left(u_{1}\right)+2 \sigma^{2}(1-\eta) \operatorname{erfi}^{2}\left(u_{2}\right)} \mathrm{d} u_{1} \mathrm{~d} u_{2} \\
& =\log (e) \int_{0}^{1} \int_{0}^{1} f_{1}\left(u_{1}, u_{2}\right) f_{2}\left(u_{1}, u_{2}\right) \mathrm{d} u_{1} \mathrm{~d} u_{2}
\end{aligned}
$$

where in (a) we interchanged expectation and differentiation (noting that the integrand is continuous for all $\eta \in[0,1])$, and we set

$$
\begin{aligned}
& f_{1}\left(u_{1}, u_{2}\right)=\operatorname{erfi}^{2}\left(u_{1}\right)-\operatorname{erfi}^{2}\left(u_{2}\right) \\
& f_{2}\left(u_{1}, u_{2}\right)=\left(1 /\left(2 \sigma^{2}\right)+\eta \operatorname{erfi}^{2}\left(u_{1}\right)+(1-\eta) \operatorname{erfi}^{2}\left(u_{2}\right)\right)^{-1} .
\end{aligned}
$$

Note that $f_{1}\left(u_{1}, u_{2}\right)$ is negative symmetric, i.e., $f_{1}\left(u_{1}, u_{2}\right)=-f_{1}\left(u_{2}, u_{1}\right)$. Furthermore, the monotonicity of $\operatorname{erfi}(u)$ implies that for $u_{1} \geq u_{2}$ we have $f_{1}\left(u_{1}, u_{2}\right) \geq 0$, and for $u_{1} \leq u_{2}$ it holds that $f_{1}\left(u_{1}, u_{2}\right) \leq 0$. We will now exploit these properties of $f_{1}\left(u_{1}, u_{2}\right)$ to complete the proof and start by rewriting the integral in (89) according to

$$
\int_{u_{1}=0}^{1} \int_{u_{2}=0}^{1} f_{1}\left(u_{1}, u_{2}\right) f_{2}\left(u_{1}, u_{2}\right) \mathrm{d} u_{1} \mathrm{~d} u_{2}
$$




$$
\begin{aligned}
& =\int_{v=0}^{1}\left(\int_{u_{1}=v}^{1} f_{1}\left(u_{1}, v\right) f_{2}\left(u_{1}, v\right) \mathrm{d} u_{1}+\int_{u_{2}=v}^{1} f_{1}\left(v, u_{2}\right) f_{2}\left(v, u_{2}\right) \mathrm{d} u_{2}\right) \mathrm{d} v \\
& =\int_{v=0}^{1} \int_{u=v}^{1} f_{1}(u, v)\left(f_{2}(u, v)-f_{2}(v, u)\right) \mathrm{d} u \mathrm{~d} v
\end{aligned}
$$

where $f_{1}(u, v) \geq 0$ in the entire range of integration since $u \geq v$. Finally, we will show that $f_{2}(u, v)-f_{2}(v, u) \geq 0$, which by (90) then implies (88). Straightforward manipulations reveal that the condition $f_{2}(u, v)-f_{2}(v, u) \geq 0$ is equivalent to

$$
\begin{gathered}
\eta \operatorname{erfi}^{2}(u)+(1-\eta) \operatorname{erfi}^{2}(v) \leq \eta \operatorname{erfi}^{2}(v)+(1-\eta) \operatorname{erfi}^{2}(u) \\
(2 \eta-1) \operatorname{erfi}^{2}(u) \leq(2 \eta-1) \operatorname{erfi}^{2}(v)
\end{gathered}
$$

and hence by $0 \leq \eta \leq 1 / 2$ to

$$
\operatorname{erfi}^{2}(u) \geq \operatorname{erfi}^{2}(v)
$$

which is satisfied for $u \geq v$ (i.e., over the entire range of integration) because erfi $(x)$ is nondecreasing.

\section{ACKNOWLEDGMENT}

We would like to thank Vinko Erceg and Pieter van Rooyen for helping both plan and making possible the measurement campaign that led to the discovery of the phase noise problem in TDMS-based MIMO channel sounding. 


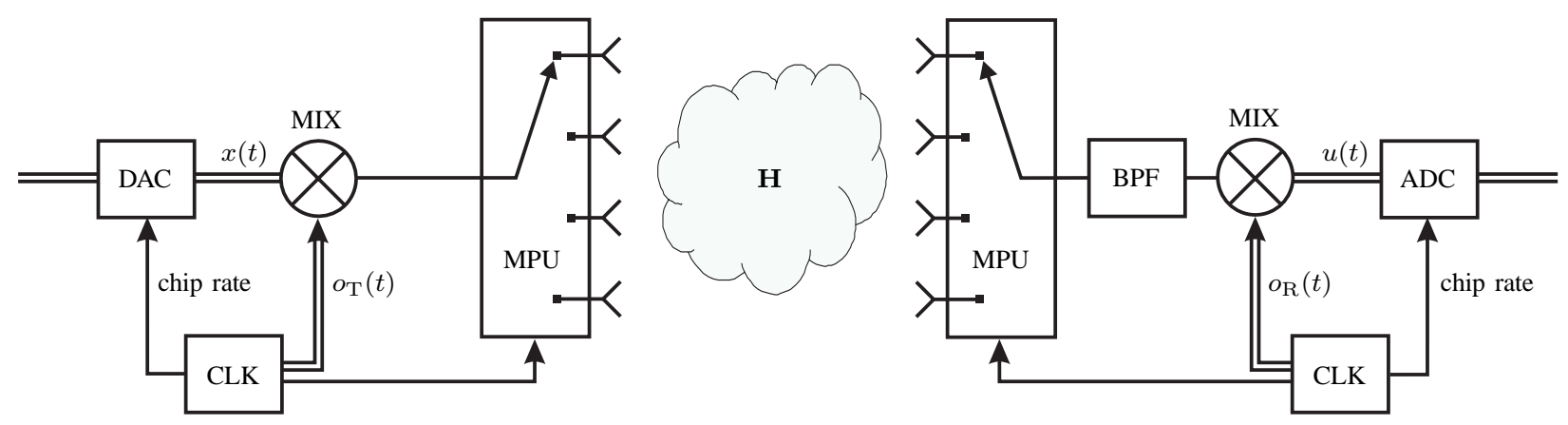

Fig. 1. Architecture of a TDMS-based MIMO channel sounder. ADC, DAC, MIX, MPU, BPF, and CLK stand for analog-todigital converter, digital-to-analog converter, mixer, multiplexing unit, bandpass filter, and (reference) clock, respectively.

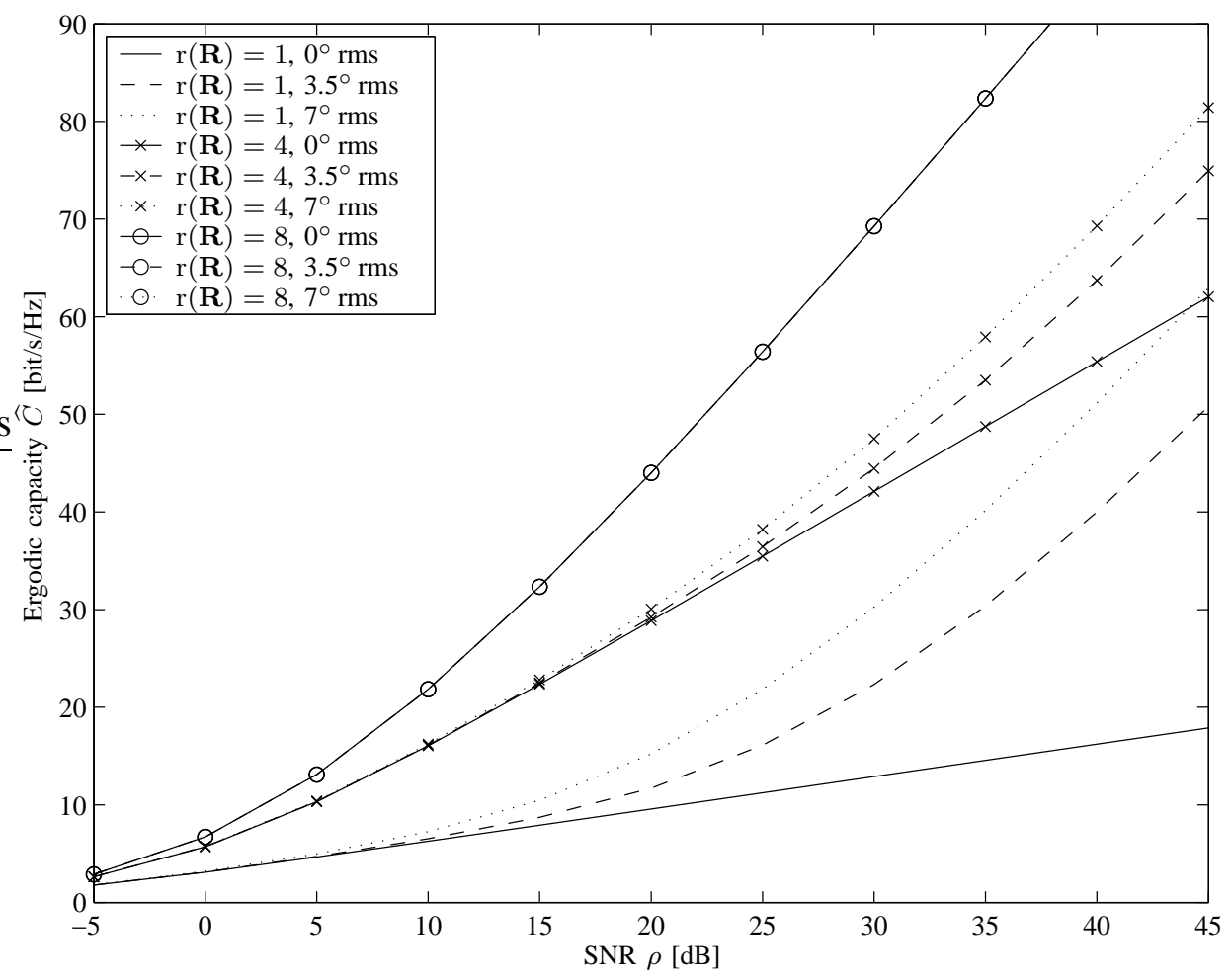

Fig. 2. Ergodic capacity of an $8 \times 8$ effective channel for fully uncorrelated phase noise with varying rms values and various $\mathrm{r}(\mathbf{R})$ of the underlying physical channel. 


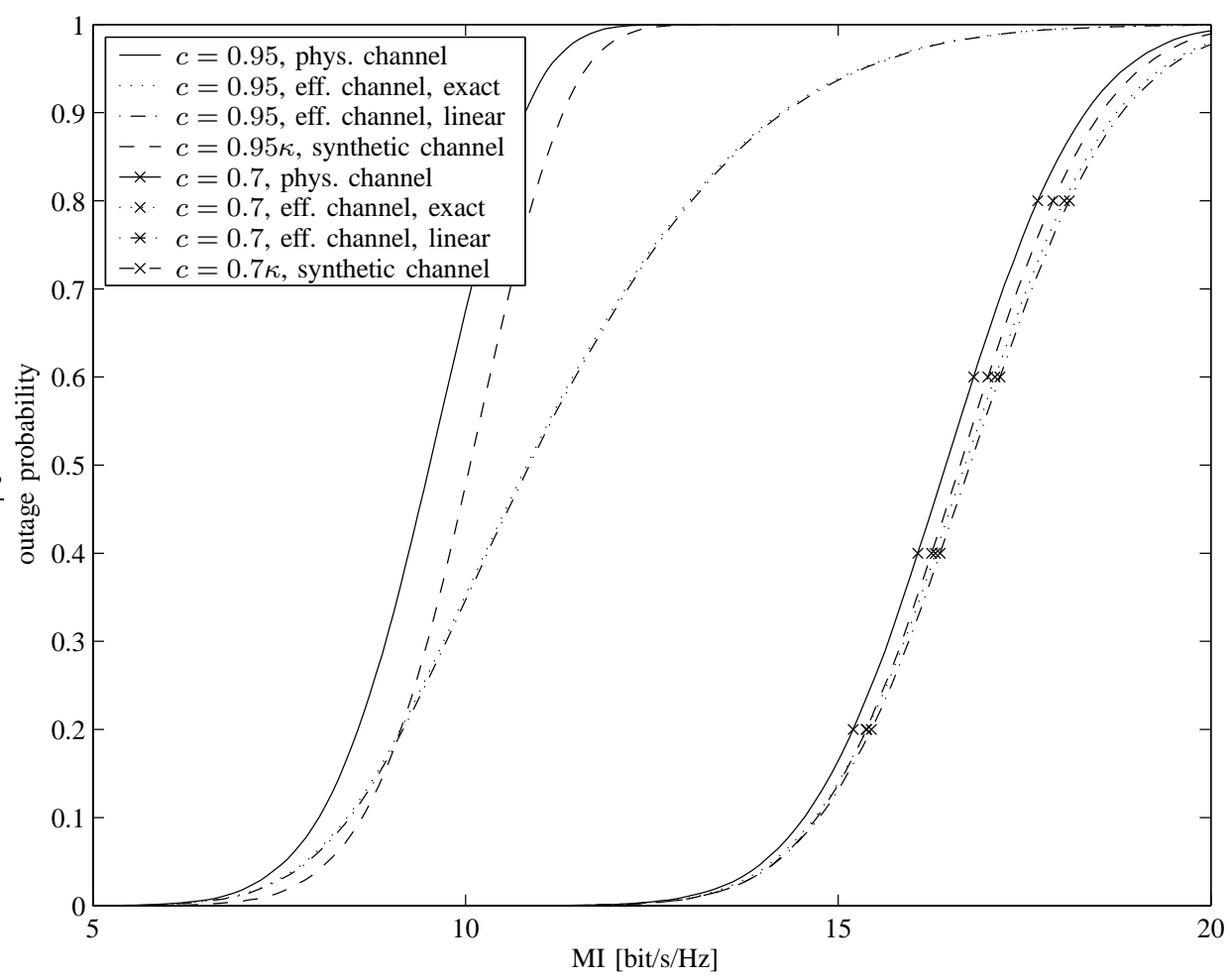

Fig. 3. cdf of mutual information at SNR $\rho=20 \mathrm{~dB}$ showing the impact of the loss of joint Gaussianity for a $4 \times 4$ physical channel with $[\operatorname{Corr}(\mathbf{H})]_{m, n}=c, \forall m \neq n$, and fully uncorrelated $7^{\circ} \mathrm{rms}$ phase noise.

TABLE I

Channel Sounder and (Two Different) Measurement Setup Parameters

\begin{tabular}{|l|c|c|}
\hline Carrier frequency & \multicolumn{2}{|c|}{$5.25 \mathrm{GHz}$} \\
Frequency generation & \multicolumn{2}{|c|}{$\begin{array}{c}\text { Rubidium reference and PLL-VCO } \\
\text { Sounding sequence }\end{array}$} \\
$\begin{array}{l}\text { Sounding pattern } \\
\text { bipolar m-sequence, rectangular chip pulses } \\
\text { regular (see Section IV-A3) }\end{array}$ \\
\hline \hline Sequence length (chips) & \multicolumn{2}{|c|}{$100 \mathrm{MHz}$} \\
MIMO configuration $\left(M_{\mathrm{R}} \times M_{\mathrm{T}}\right)$ & $16 \times 16$ & 31 \\
MIMO snapshot distance $T_{\mathrm{MIMO}}$ & $19.46 \mathrm{~ms}$ & $23 \times 23$ \\
Receive-side SISO snapshot distance $T_{\mathrm{R}}$ & $10.22 \mu \mathrm{s}$ & $0.27 \mathrm{~ms}$ \\
Ratio $T_{\mathrm{T}} / T_{\mathrm{R}}$ & 17 & 24 \\
SISO snapshot duration $\left|\mathfrak{I}_{\mathrm{P}}\right|$ & $5.11 \mu \mathrm{s}$ & $0.31 \mu \mathrm{s}$ \\
\hline
\end{tabular}




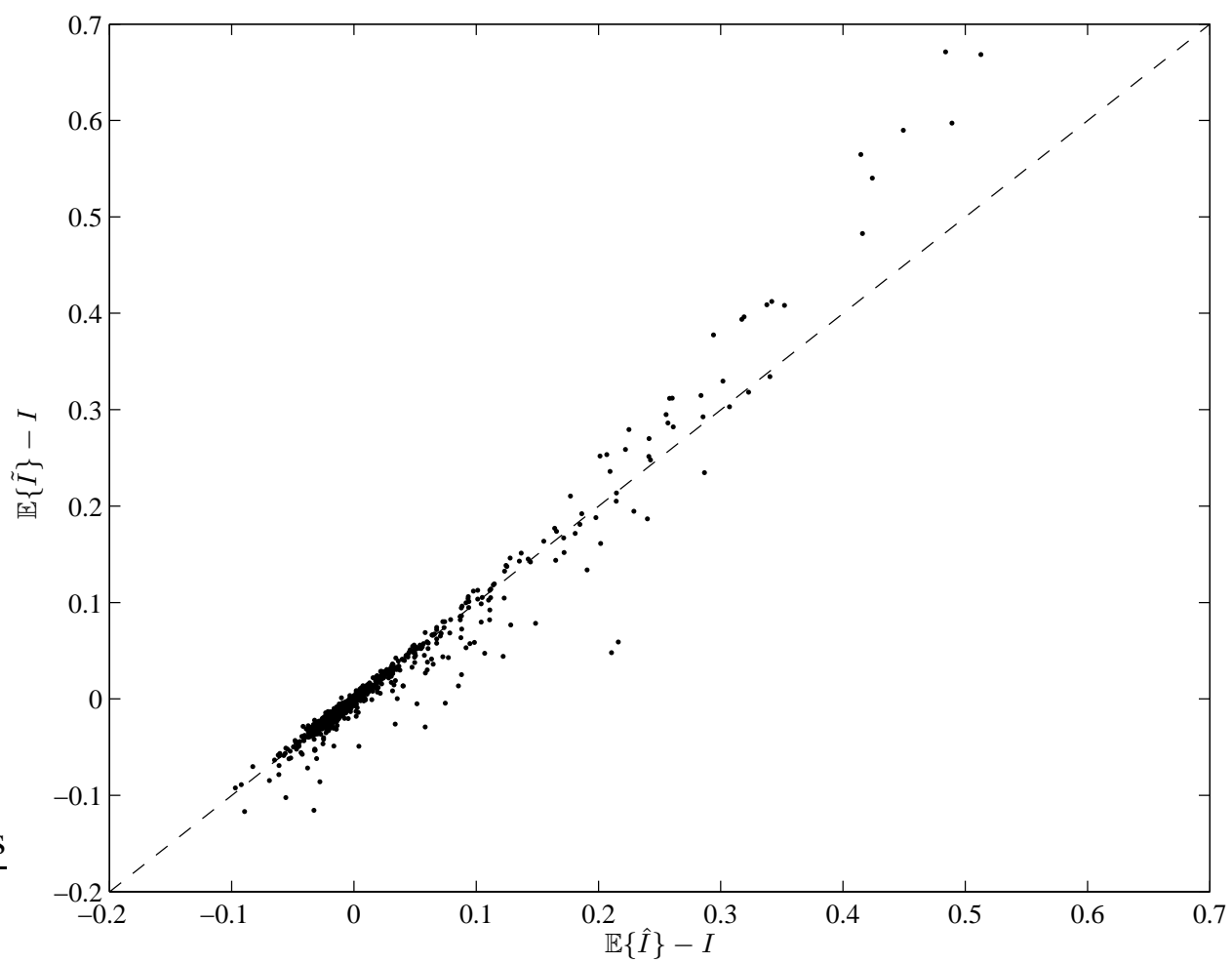

(a) First moment of $\hat{I}$ and of second order Taylor series expansion $\tilde{I}$

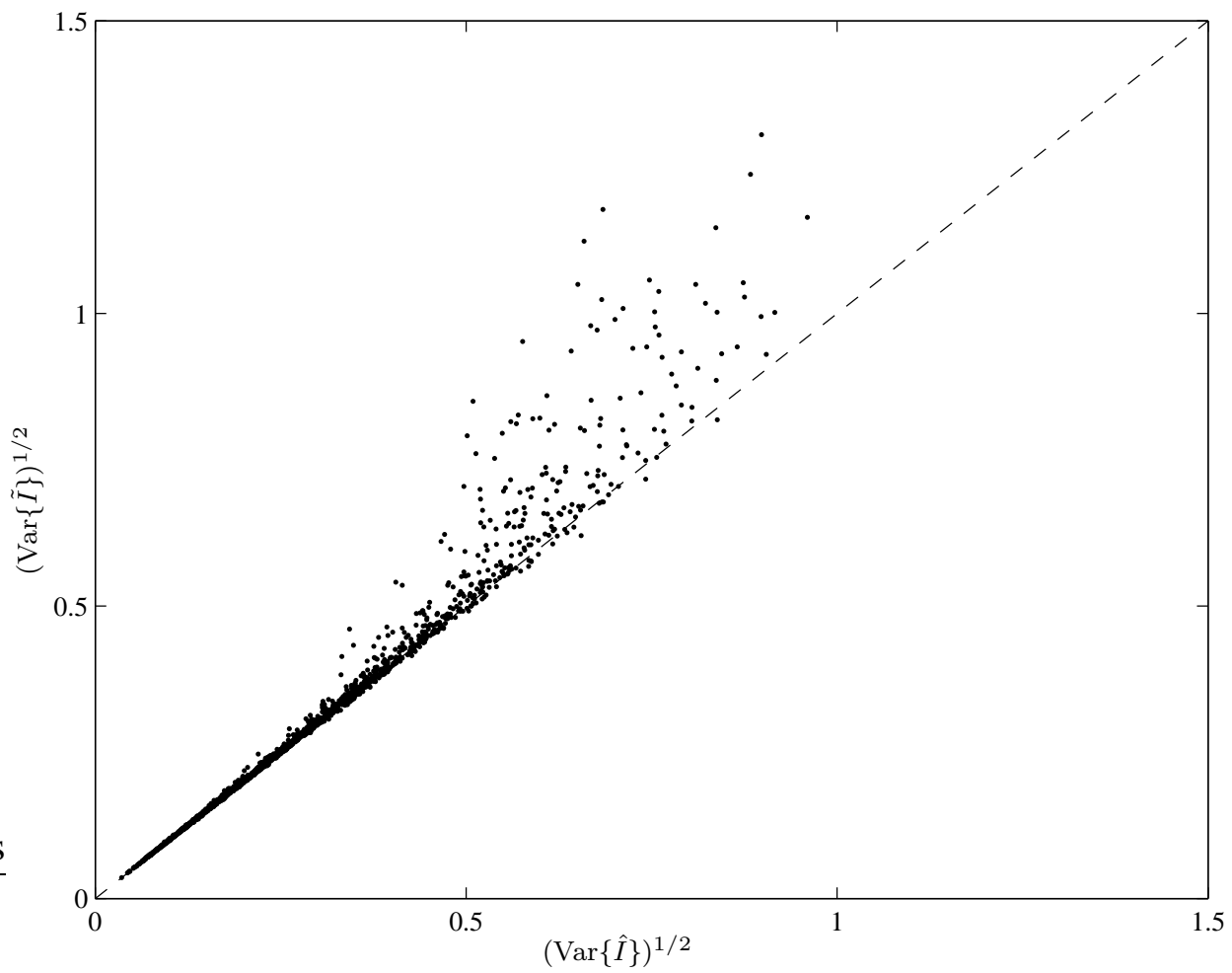

(b) Second moment of $\hat{I}$ and of second order Taylor series expansion $\tilde{I}$

Fig. 4. Moments of mutual information $\hat{I}$ and of mutual information second order Taylor series expansion $\tilde{I}$, both for 2000 realizations of an i.i.d. Gaussian physical channel subject to fully uncorrelated $3.5^{\circ} \mathrm{rms}$ phase noise at SNR $\rho=30 \mathrm{~dB}$. Moments of $\hat{I}$ are obtained from Monte Carlo simulation over 10000 phase noise samples per physical channel realization. 


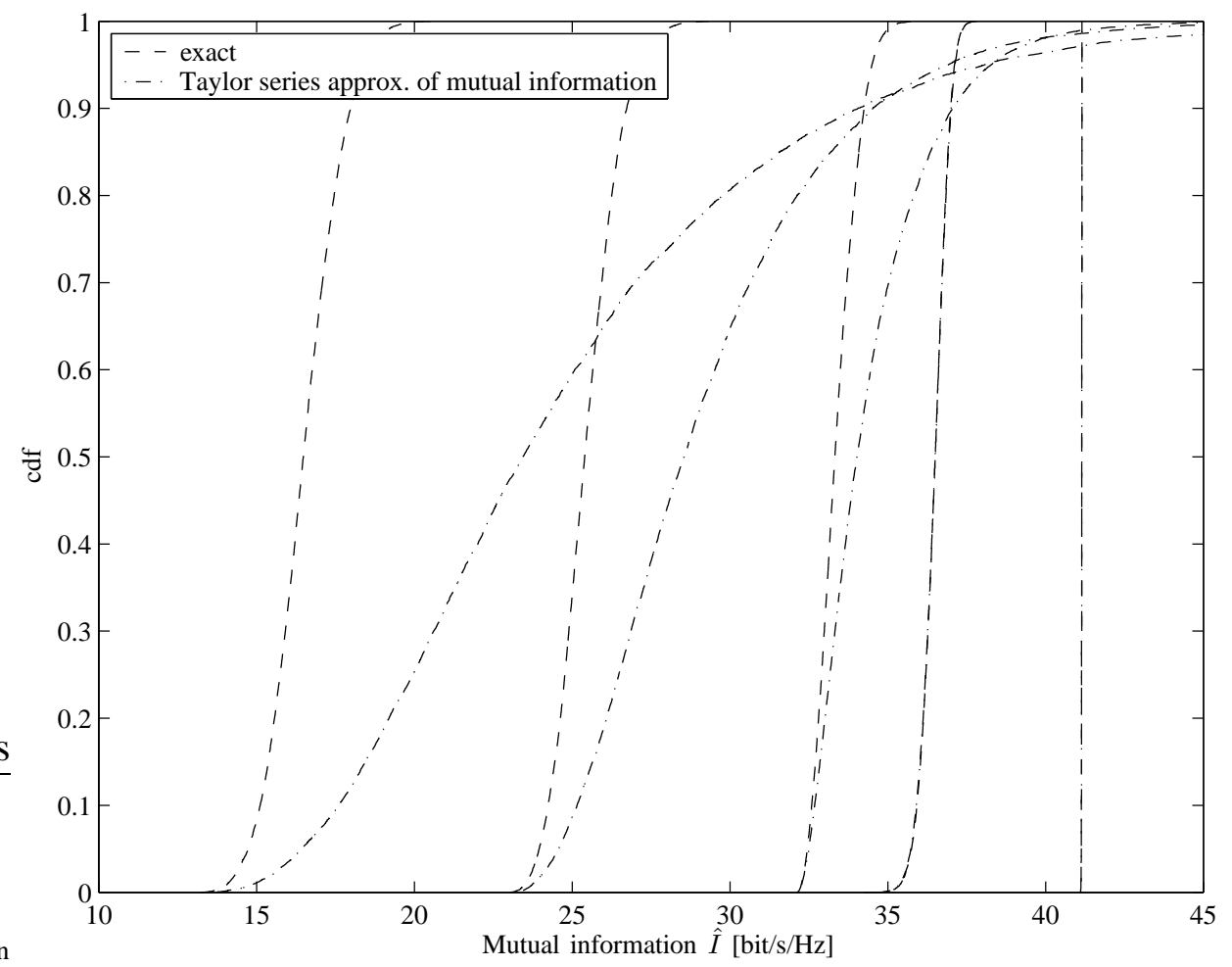

(a) cdfs corresponding to the second-order Taylor series approximation of mutual information

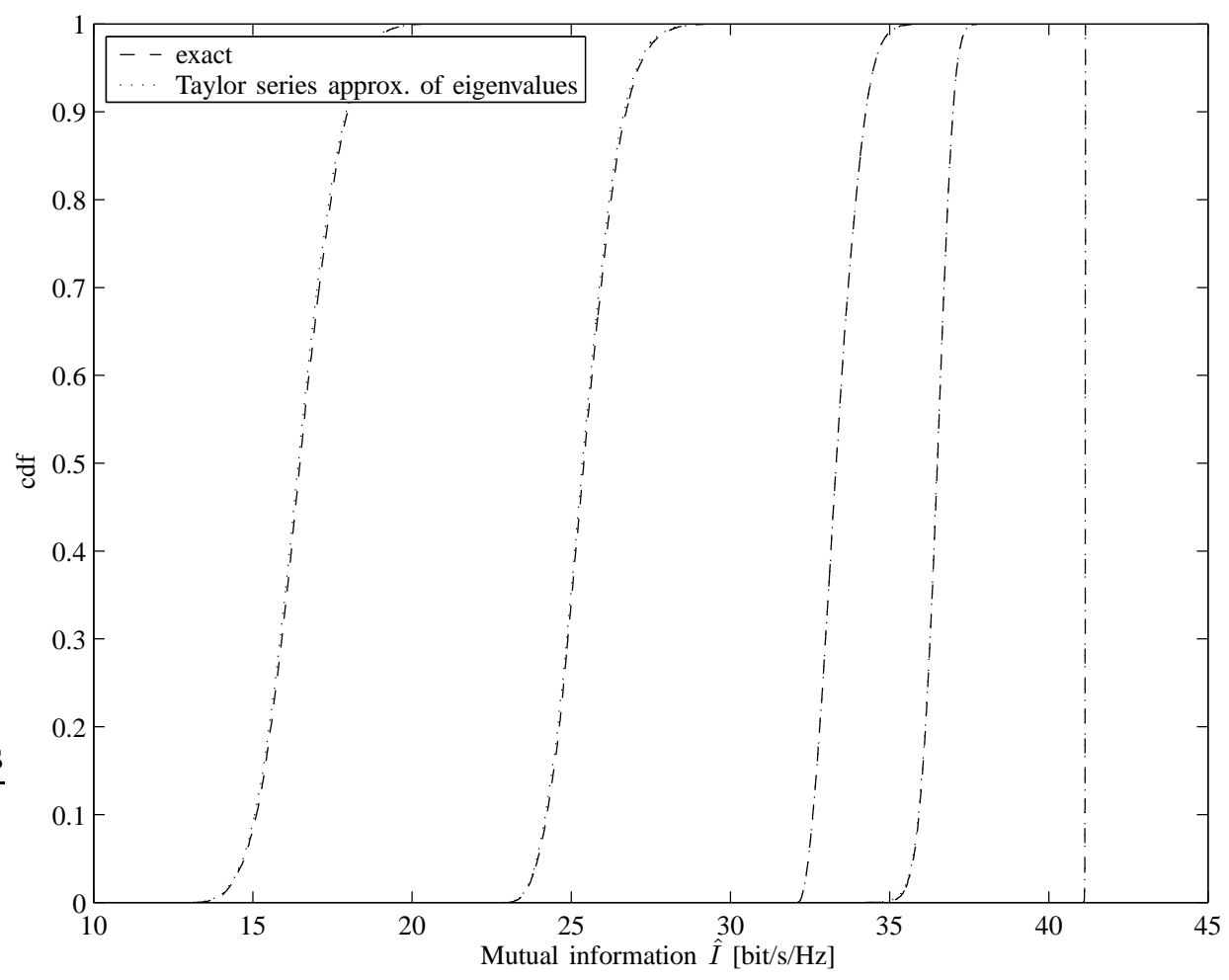

(b) cdfs corresponding to second-order Taylor series approximation of channel eigenvalues

Fig. 5. cdfs of mutual information and of corresponding approximation based on second-order Taylor series expansion (27), as well as second-order Taylor series approximation of eigenvalues, for realizations of a (from left to right) rank-1, balanced rank-2, balanced rank-3, rank- 4 , and balanced rank- 4 physical $4 \times 4$ channel subject to fully uncorrelated $3.5^{\circ}$ rms phase noise (10000 realizations) at $\operatorname{SNR} \rho=30 \mathrm{~dB}$. For all channels, we employed the normalization $\|\mathbf{H}\|_{\mathrm{F}}^{2}=M_{\mathrm{T}} M_{\mathrm{R}}$. 


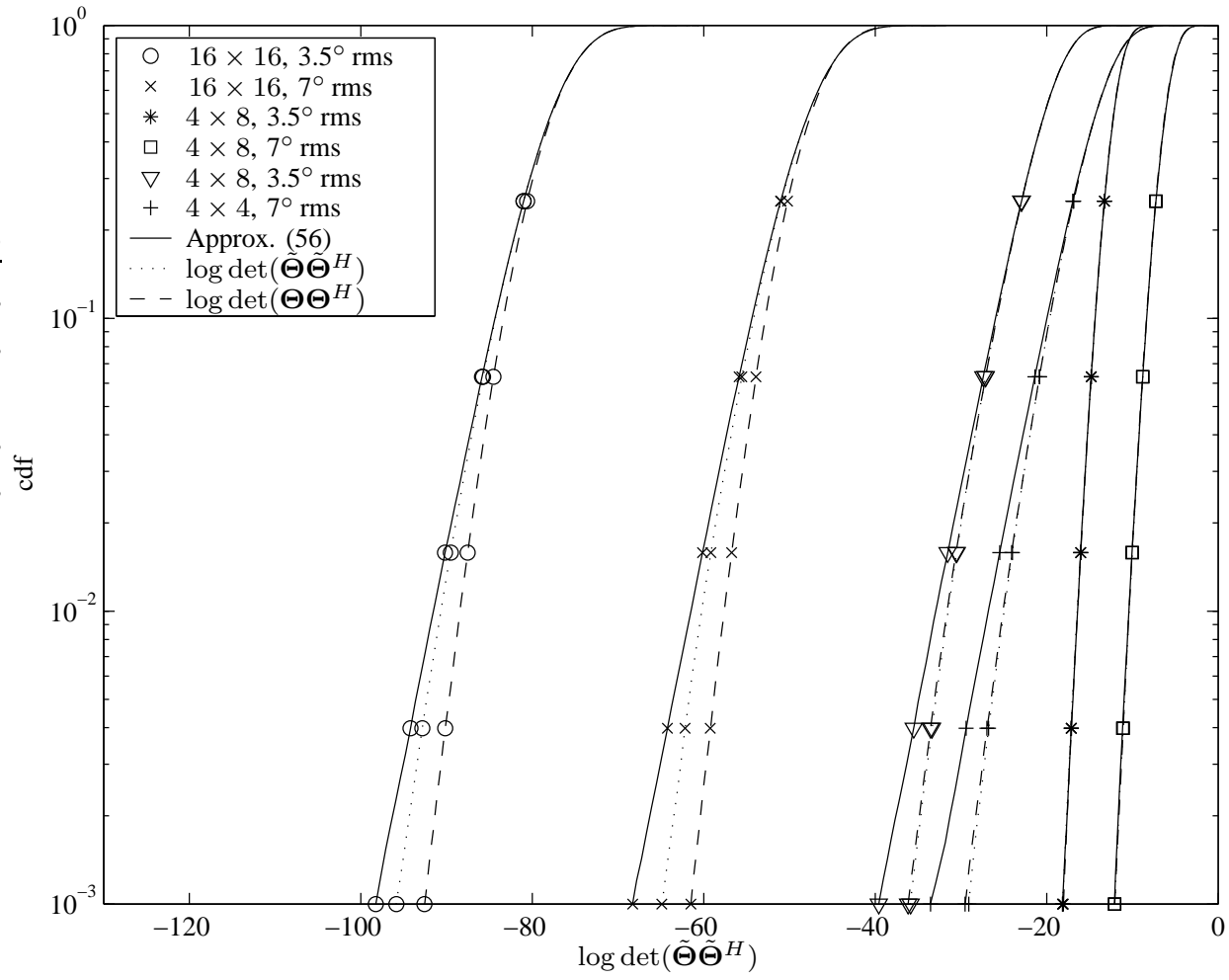

Fig. 6. $\operatorname{cdf}$ of $\log \operatorname{det}\left(\tilde{\Theta} \tilde{\Theta}^{H}\right)$ and analytic approximation $\left(56\right.$, for various $M_{\mathrm{T}}, M_{\mathrm{R}}$ and different phase noise levels. For comparison, $\log \operatorname{det}\left(\Theta \Theta^{H}\right)$ is also shown. A regular sounding pattern according to 26) was assumed. All results are based on 300000 Monte Carlo runs. 


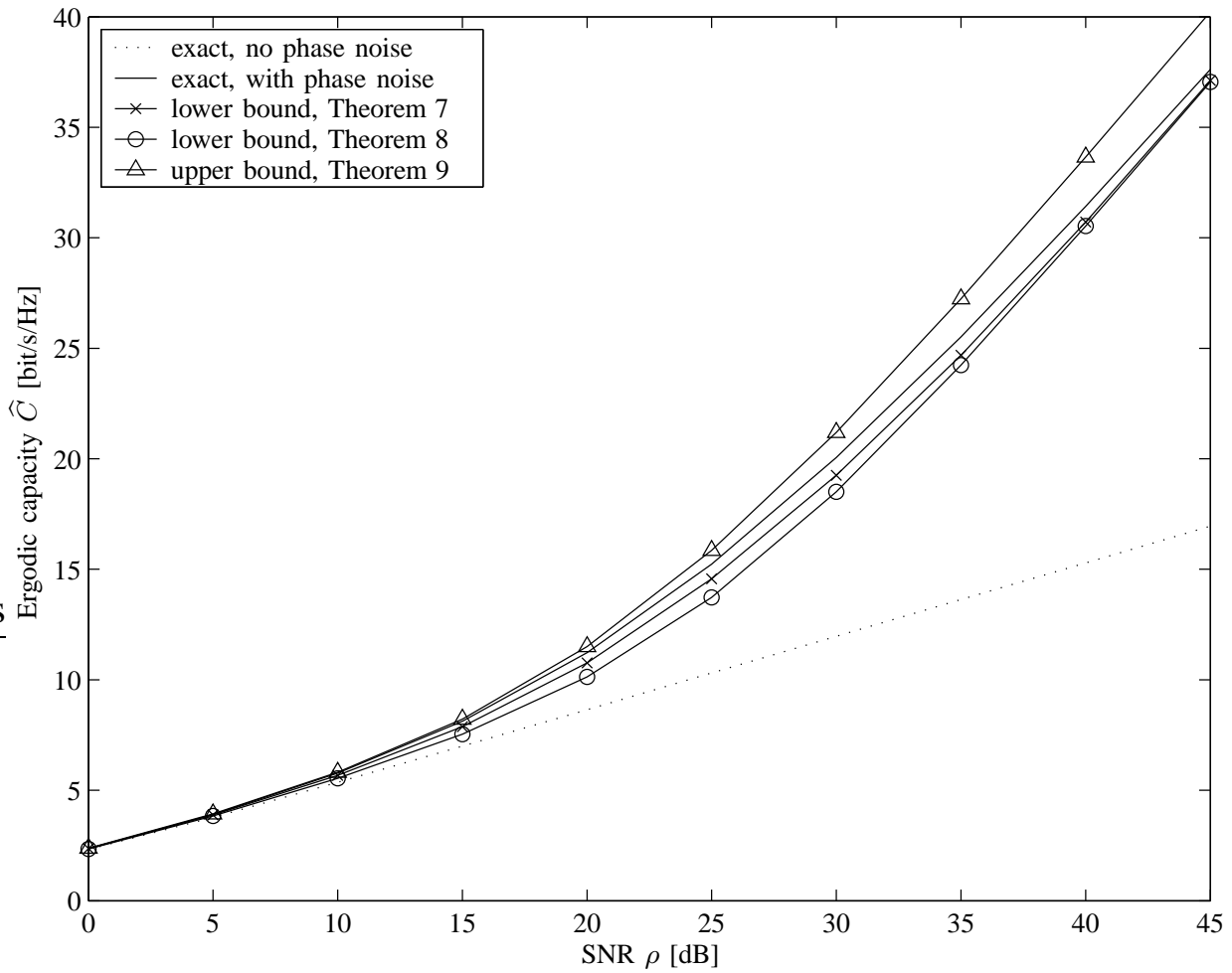

Fig. 7. Ergodic capacity of a $4 \times 4$ rank-1 physical channel with unit-modulus entries subject to $7^{\circ}$ rms fully uncorrelated phase noise. Exact results are obtained through Monte Carlo simulation. 


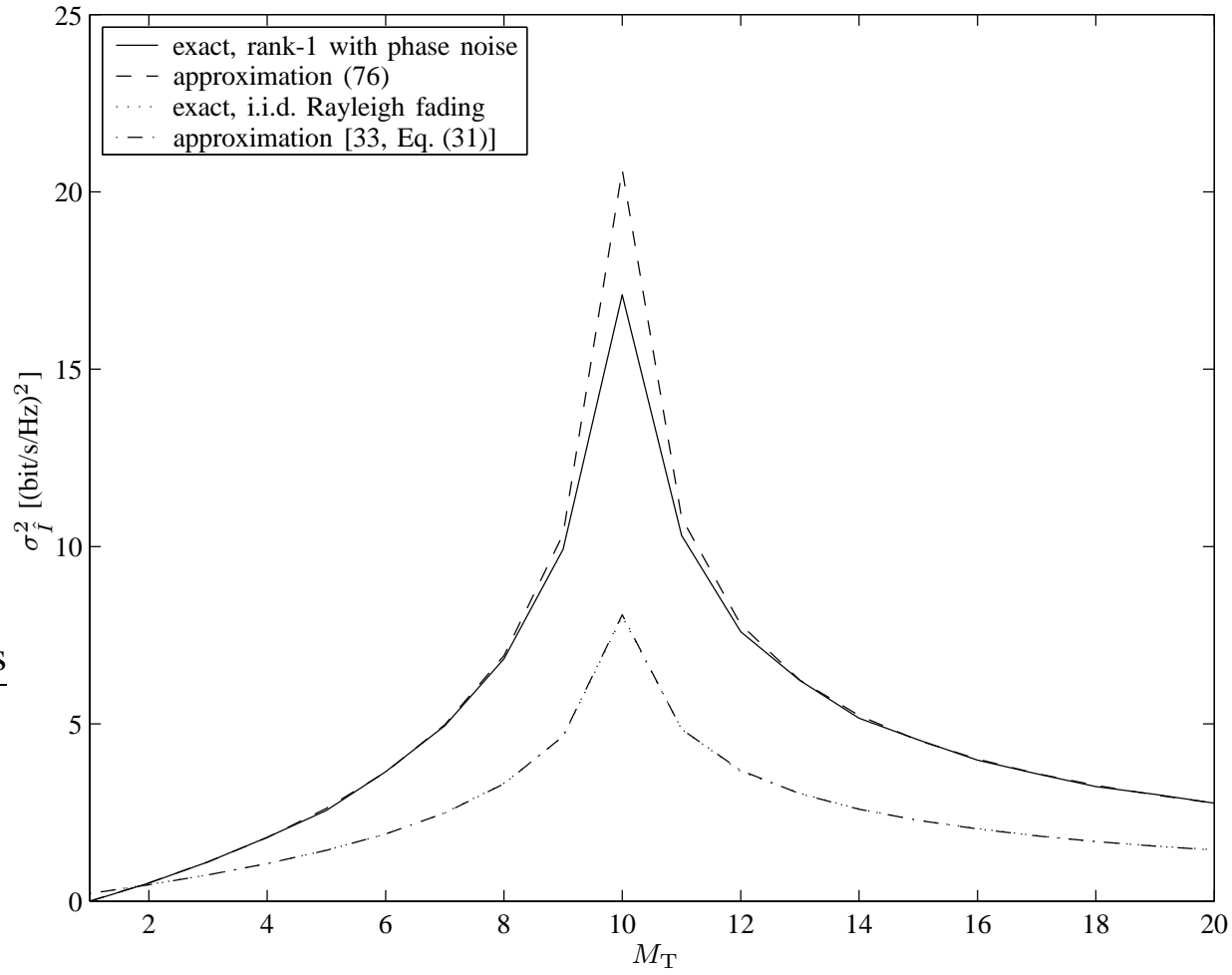

Fig. 8. Exact variance of mutual information and analytic approximation (76) at high $\mathrm{SNR}$ for $M_{\mathrm{R}}=10$ as a function of $M_{\mathrm{T}}$ for a rank-1 physical channel with unit-modulus entries subject to $3.5^{\circ} \mathrm{rms}$ fully uncorrelated phase noise, and for an i.i.d. Rayleigh fading channel. Exact results are obtained through Monte Carlo simulation. 


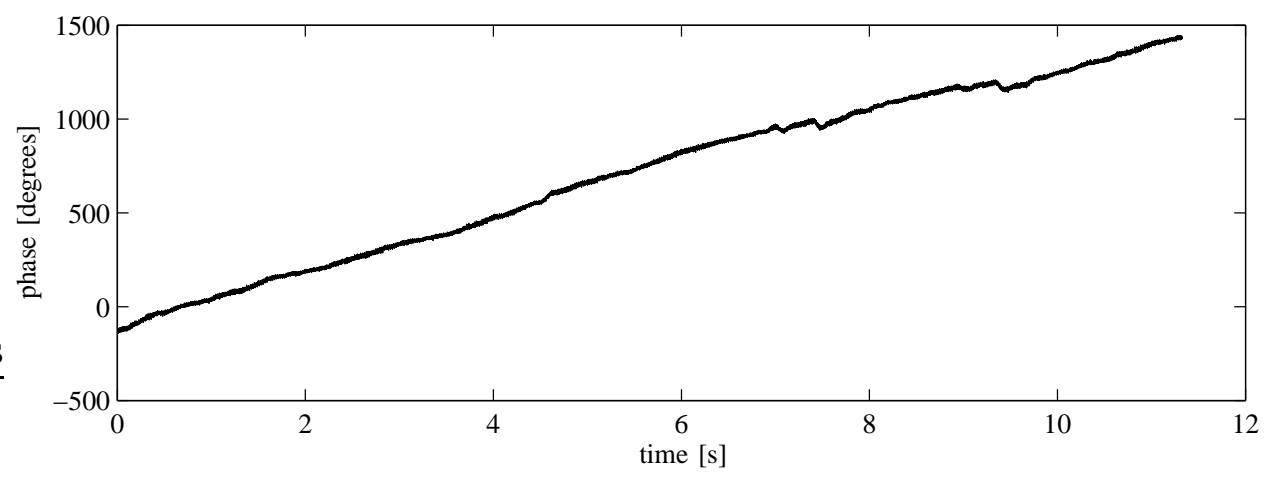

(a) Phase characteristics

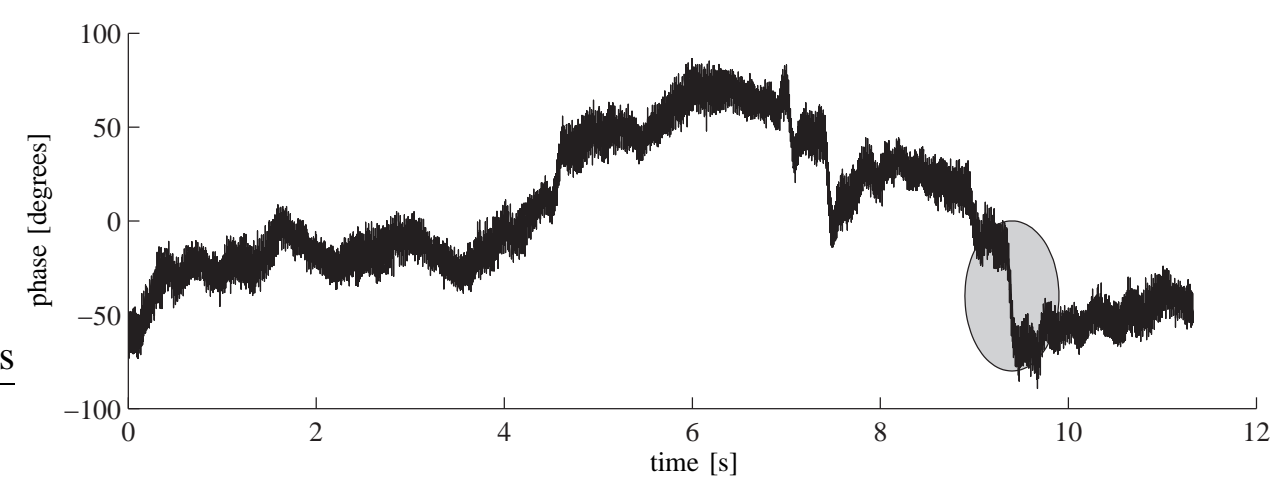

(b) Phase characteristics after removal of estimated linear component

Fig. 9. Long-term phase noise behavior of a commercially employed TDMS-based MIMO channel sounder (length-31 sounding sequence). 


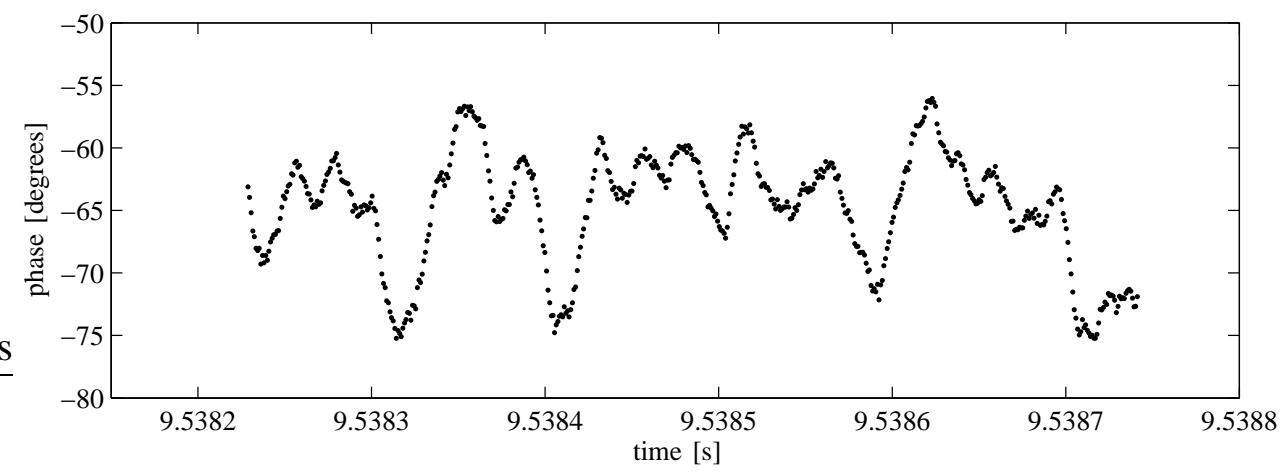

(a) Phase-noise trace of a single MIMO snapshot (length-31 sounding sequence)

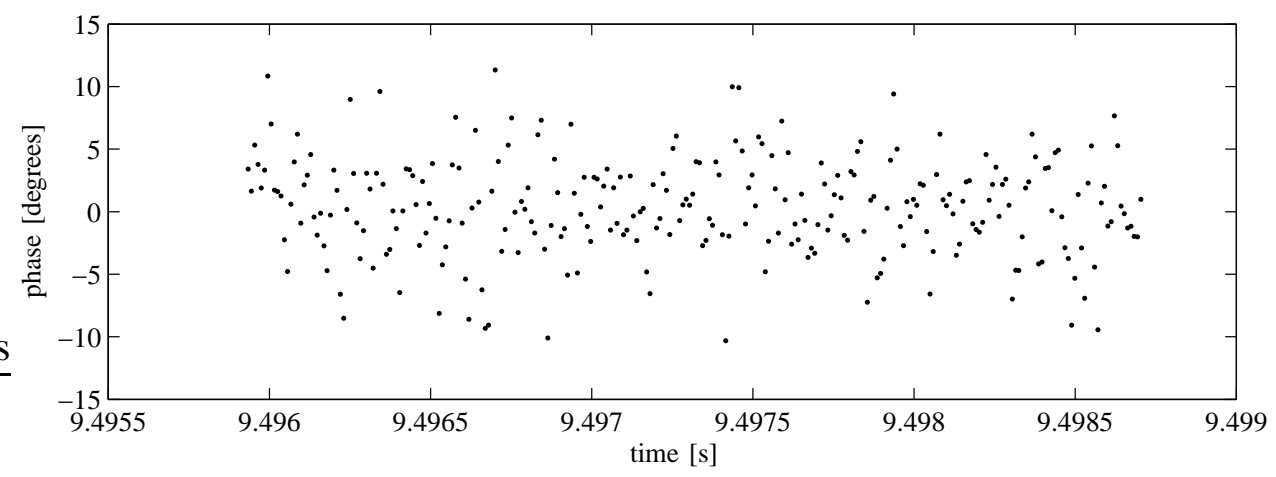

(b) Phase-noise trace of a single MIMO snapshot (length-511 sounding sequence)

Fig. 10. Short-term phase noise behavior of a commercially employed TDMS-based MIMO channel sounder. 


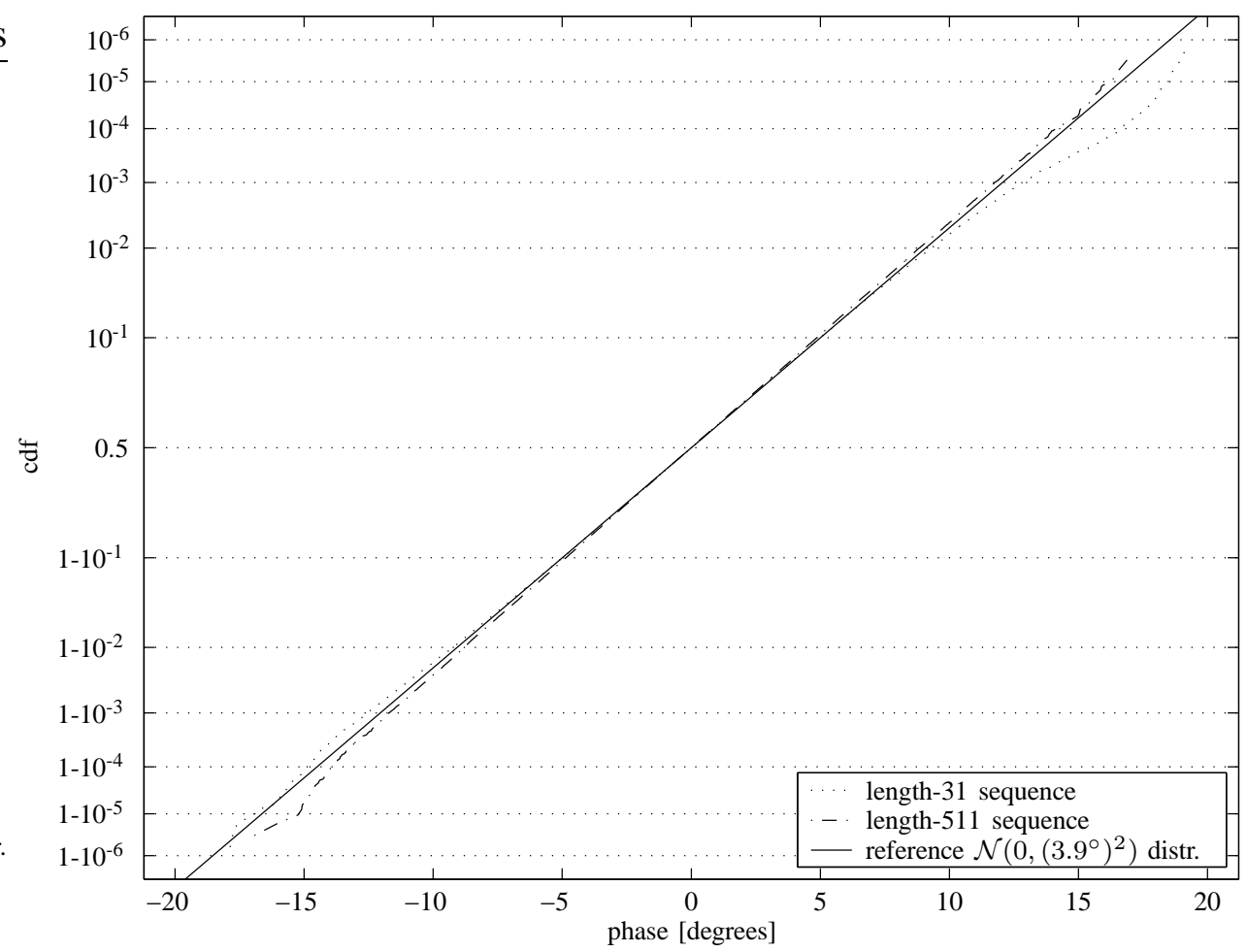

Fig. 11. cdf of the measured MIMO snapshot phase noise traces (i.e., overall phase after removal of estimated linear component) compared to $\mathcal{N}\left(0,\left(3.9^{\circ}\right)^{2}\right)$ reference distribution.

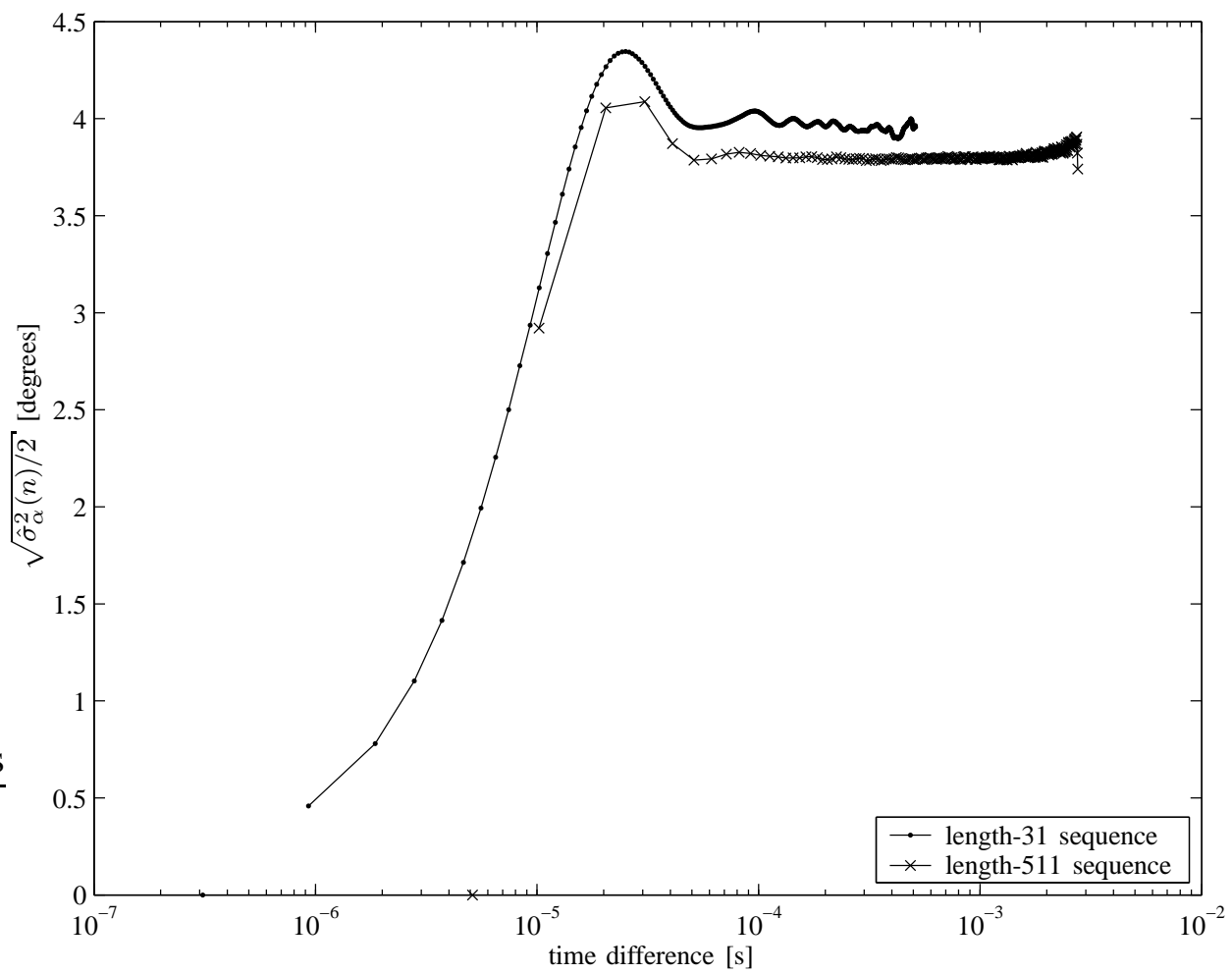

Fig. 12. $\sqrt{\hat{\sigma}_{\alpha}^{2}(n) / 2}$ for length-31 and length-511 sounding sequences. 


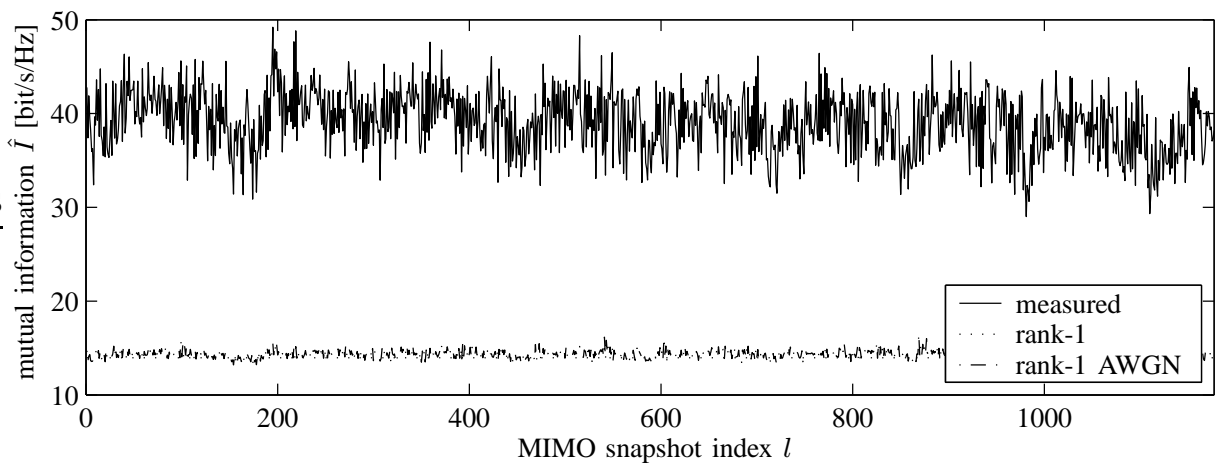

(a) Mutual information for measured channel and for reference rank-1 physical channel

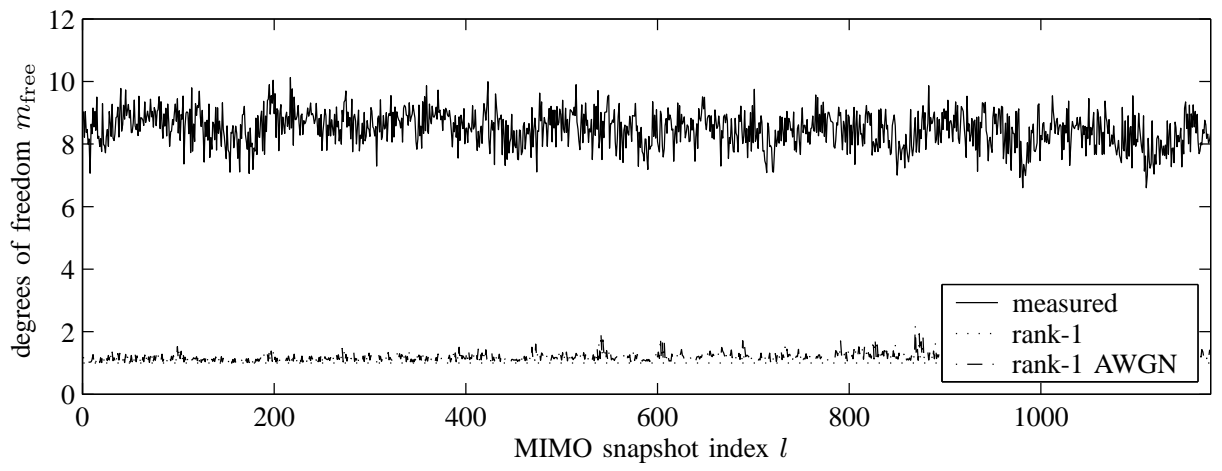

(b) Number of degrees of freedom $m_{\text {free }}$ corresponding to results in (a)

Fig. 13. Mutual information and corresponding number of degrees of freedom $m_{\text {free }}$ at SNR $\rho=30 \mathrm{~dB}$ for $M_{\mathrm{T}}=M_{\mathrm{R}}=16$ configuration of Table $\prod$ and a rank-1 "calibration measurement" conducted on a commercially employed TDMS-based MIMO channel sounder. For reference, we also show the mutual information corresponding to the rank-1 physical channel $\mathbf{H}=\mathbf{1}$ and the mutual information corresponding to a rank-1 physical channel subject to AWGN on the channel coefficients (see Section VII-C). 


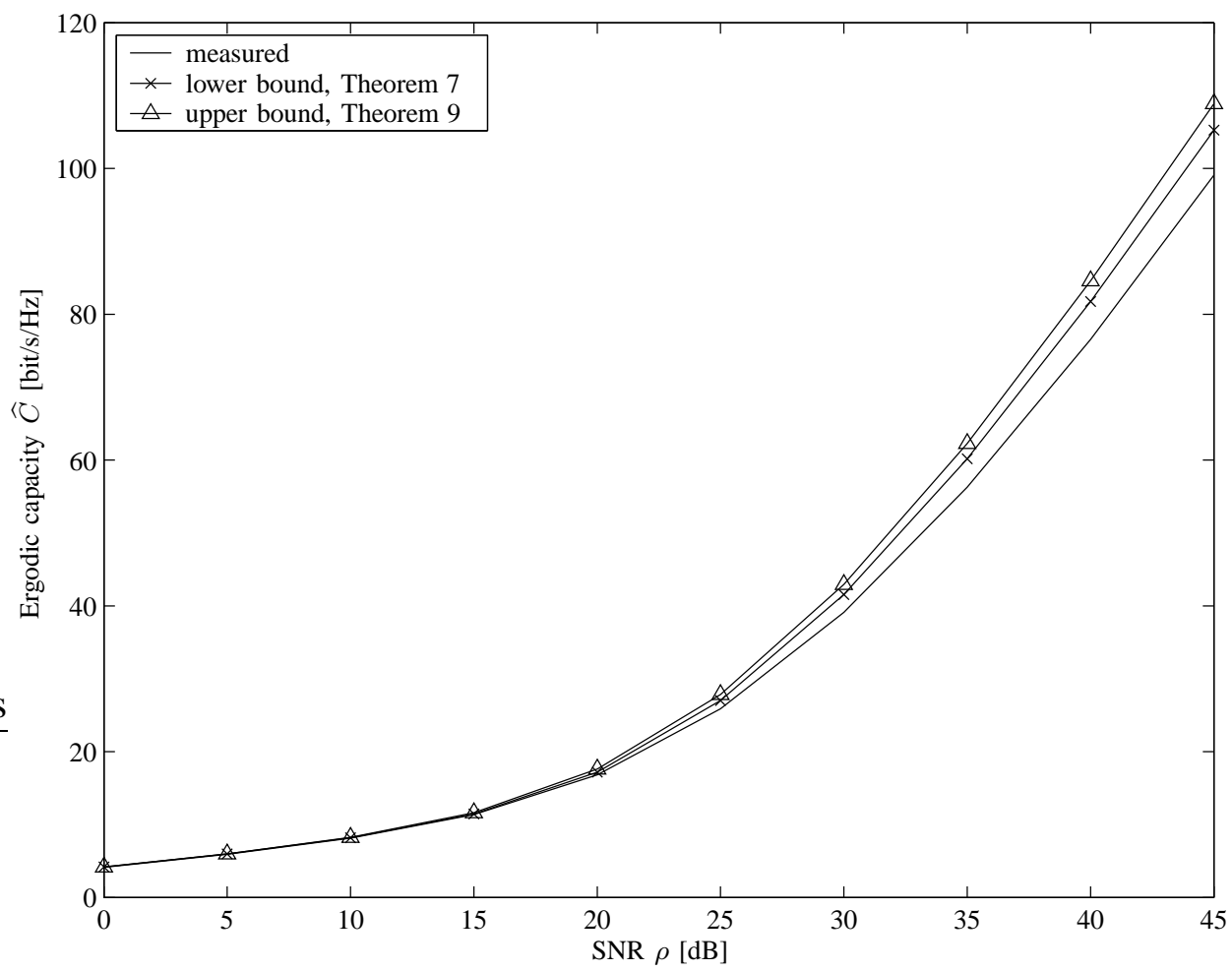

Fig. 14. Measured ergodic capacity along with corresponding analytic lower and upper bound in Theorems 7 and 9 , respectively. 


\section{REFERENCES}

[1] G. Matz, A. F. Molisch, F. Hlawatsch, M. Steinbauer, and I. Gaspard, "On the systematic measurement error of correlative mobile radio channel sounders," IEEE Trans. Commun., vol. 50, no. 5, pp. 808-821, May 2002.

[2] D. Gesbert, H. Bölcskei, D. A. Gore, and A. J. Paulraj, "Outdoor MIMO wireless channels: Models and performance prediction," IEEE Trans. Commun., vol. 50, no. 12, pp. 1926-1934, Dec. 2002.

[3] D. Chizhik, G. J. Foschini, M. J. Gans, and R. A. Valenzuela, "Keyholes, correlations, and capacities of multielement transmit and receive antennas," IEEE Trans. Wireless Commun., vol. 1, no. 2, pp. 361-368, Apr. 2002.

[4] J. Kivinen and P. Vainikainen, "Phase noise in a direct sequence based channel sounder," in Proc. IEEE Int. Symp. on Personal, Indoor \& Mobile Radio Commun. (PIMRC), vol. 3, Helsinki, Finland, Sep. 1997, pp. 1115-1119.

[5] _ _ "Calibration scheme for synthesizer phase fluctuations in virtual antenna array measurements," Microwave \& Optical Technol. Lett., vol. 26, no. 3, pp. 183-187, Jun. 2000.

[6] J. A. Fessler and A. O. Hero, "Space-alternating generalized expectation-maximization algorithm," IEEE Trans. Signal Processing, vol. 42, no. 10, pp. 2664-2677, Oct. 1994.

[7] P. Almers, S. Wyne, F. Tufvesson, and A. F. Molisch, "Effect of random walk phase noise on MIMO measurements," in Proc. IEEE Veh. Technol. Conf. (VTC) Spring, vol. 1, Stockholm, Sweden, May 2005, pp. 141-145.

[8] M. J. Gans, N. Amitay, Y. S. Yeh, H. Xu, T. C. Damen, R. A. Valenzuela, T. Sizer, R. Storz, D. Taylor, W. M. MacDonald, C. Tran, and A. Adamiecki, "Outdoor BLAST measurement system at $2.44 \mathrm{GHz}$ : Calibration and initial results," IEEE J. Select. Areas Commun., vol. 20, no. 3, pp. 570-583, Apr. 2002.

[9] P. Kyritsi, R. A. Valenzuela, and D. C. Cox, "Channel and capacity estimation errors," IEEE Commun. Lett., vol. 6, no. 12, pp. 517-519, Dec. 2002.

[10] S. Loyka and A. Kouki, "On MIMO channel capacity, correlations, and keyholes: Analysis of degenerate channels," IEEE Trans. Commun., vol. 50, no. 12, pp. 1886-1888, Dec. 2002.

[11] P. Almers, F. Tufvesson, and A. F. Molisch, "Measurement of keyhole effect in a wireless multiple-input multiple-output (MIMO) channel," IEEE Commun. Lett., vol. 7, no. 8, pp. 373-375, Aug. 2003.

[12] J. R. Magnus and H. Neudecker, Matrix Differential Calculus with Applications in Statistics and Econometrics, 2nd ed., ser. Wiley Series in Probability \& Statistics. Chichester, UK: Wiley-VCH, 1999.

[13] F. D. Neeser and J. L. Massey, "Proper complex random processes with applications to information theory," IEEE Trans. Inform. Theory, vol. 39, no. 4, pp. 1293-1302, Jul. 1993.

[14] K.-T. Fang, S. Kotz, and K.-W. Ng, Symmetric Multivariate and Related Distributions, ser. Monographs on Statistics and Applied Probability. London, UK and New York, NY: Chapman and Hall, 1990.

[15] M. Abramowitz and I. A. Stegun, Eds., Handbook of Mathematical Functions with Formulas, Graphs, and Mathematical Tables, 9th ed. New York, NY: Dover, 1972.

[16] G. Matz, A. F. Molisch, M. Steinbauer, F. Hlawatsch, I. Gaspard, and H. Artés, "Bounds on the systematic measurement 
error of channel sounders for time-varying mobile radio channels," in Proc. IEEE Veh. Technol. Conf. (VTC) Fall, vol. 3, Amsterdam, The Netherlands, Sep. 1999, pp. 1465-1470.

[17] P. G. Flikkema and S. G. Johnson, "A comparison of time- and frequency-domain wireless channel sounding techniques," in Proc. IEEE SoutheastCon, Tampa, FL, Apr. 1996, pp. 488-491.

[18] P. J. Cullen, P. C. Fannin, and A. Molina, "Wide-band measurement and analysis techniques for the mobile radio channel," IEEE Trans. Veh. Technol., vol. 42, no. 4, pp. 589-603, Nov. 1993.

[19] J. D. Parsons, D. A. Demery, and A. M. D. Turkmani, "Sounding techniques for wideband mobile radio channels: A review," Proc. IEE Part I: Commun., Speech \& Vision, vol. 138, no. 5, pp. 437-446, Oct. 1991.

[20] J. Rutman, "Characterization of phase and frequency instabilities in precision frequency sources: Fifteen years of progress," Proc. IEEE, vol. 66, no. 9, pp. 1048-1075, Sep. 1978.

[21] J. Rutman and F. L. Walls, "Characterization of frequency stability in precision frequency sources," Proc. IEEE, vol. 79, no. 7, pp. 952-960, Jul. 1991.

[22] E. A. Gerber and A. Ballato, Eds., Precision Frequency Control: Acoustic Resonators and Filters (Volume 1), Oscillators and Standards (Volume 2). New York, NY: Academic Press, 1985.

[23] A. Demir, A. Mehrotra, and J. Roychowdhury, "Phase noise in oscillators: A unifying theory and numerical methods for characterization," IEEE Trans. Circuits Syst., vol. 47, no. 5, pp. 655-674, May 2000.

[24] A. Demir, "Phase noise and timing jitter in oscillators with colored-noise sources," IEEE Trans. Circuits Syst., vol. 49, no. 12, pp. 1782-1791, Dec. 2002.

[25] A. Mehrotra, “Noise analysis of phase-locked loops," IEEE Trans. Circuits Syst., vol. 49, no. 9, pp. 1309-1316, Sep. 2002.

[26] (2006) IEEE ultrasonics, ferroelectrics, and frequency control society. [Online]. Available: http://www.ieee-uffc.org/

[27] P. M. Woodward, Probability and Information Theory with Application to Radar. London, UK: Pergamon Press, 1953.

[28] G. Sommerkorn, A. Richter, R. S. Thomä, and W. Wirnitzer, “Antenna multiplexing and time alignment for MIMO channel sounding," in Proc. Int. Union of Radio Science (URSI) General Assembly (GA), Maastricht, The Netherlands, Aug. 2002, CP.P.28, paper no. 1879 .

[29] A. Hajimiri and T. H. Lee, The Design of Low Noise Oscillators. Dordrecht, The Netherlands: Kluwer Academic Publishers, 1999.

[30] R. A. Wooding, “The multivariate distribution of complex normal variables,” Biometrika, vol. 43, no. 1/2, pp. 212-215, Jun. 1956.

[31] I. E. Telatar, "Capacity of multi-antenna Gaussian channels," Eur. Trans. Telecommun., vol. 10, no. 6, pp. 585-595, Nov. 1999.

[32] D.-S. Shiu, G. J. Foschini, M. J. Gans, and J. M. Kahn, "Fading correlation and its effect on the capacity of multielement antenna systems," IEEE Trans. Commun., vol. 48, no. 3, pp. 502-513, Mar. 2000.

[33] Ö. Oyman, R. U. Nabar, H. Bölcskei, and A. J. Paulraj, "Characterizing the statistical properties of mutual information in MIMO channels," IEEE Trans. Signal Processing, vol. 51, no. 11, pp. 2784-2795, Nov. 2003. 
[34] G. Alfano, A. Lozano, A. M. Tulino, and S. Verdú, "Mutual information and eigenvalue distribution of MIMO Ricean channels," in Proc. IEEE Int. Symp. on Inform. Theory \& Appl. (ISITA), Parma, Italy, Oct. 2004, pp. 1040-1045.

[35] Q. T. Zhang, X. W. Cui, and X. M. Li, "Very tight capacity bounds for MIMO-correlated Rayleigh-fading channels," IEEE Trans. Wireless Commun., vol. 4, no. 2, pp. 681- 688, Mar. 2005.

[36] A. M. Tulino, A. Lozano, and S. Verdú, "Impact of antenna correlation on the capacity of multiantenna channels," IEEE Trans. Inform. Theory, vol. 51, no. 7, pp. 2491-2509, Jul. 2005.

[37] M. Kang and M. S. Alouini, "Capacity of correlated MIMO Rayleigh channels," IEEE Trans. Wireless Commun., vol. 5, no. 1, pp. 143-155, Jan. 2006.

[38] W. C. Jakes, Jr., "A comparison of specific space diversity techniques for reduction of fast fading in UHF mobile radio systems," IEEE Trans. Veh. Technol., vol. 20, no. 4, pp. 81-92, Nov. 1971.

[39] W. Lee, "Effects on correlation between two mobile radio base-station antennas," IEEE Trans. Commun., vol. 21, no. 11, pp. 1214-1224, Nov. 1973.

[40] K. Kreutz-Delgado, "The complex gradient operator and the $\mathbb{C} R$-calculus," Lecture Supplement to ECE275A, Ver. ECE275CG-F05v1.3c, University of California, San Diego, USA, 2005. [Online]. Available: http://dsp.ucsd.edu/ kreutz/PEI05.html

[41] A. M. Mathai and S. B. Provost, Quadratic Forms in Random Variables: Theory and Applications. New York, NY: M. Dekker, 1992.

[42] M. Torki, "Second-order directional derivatives of all eigenvalues of a symmetric matrix," Nonlinear Anal.: Theory, Methods \& Applications, vol. 46, no. 8, pp. 1133-1150, Dec. 2001.

[43] R. A. Horn and C. R. Johnson, Matrix Analysis. Cambridge, UK: Cambridge University Press, 1985.

[44] M. L. Eaton and M. D. Perlman, "The non-singularity of generalized sample covariance matrices," Ann. Stat., vol. 1, no. 4, pp. 710-717, Jul. 1973.

[45] M. Capiński and P. E. Kopp, Measure, Integral and Probability, 2nd ed. London, UK: Springer, 1999.

[46] T. McKelvey, H. Akçay, and L. Ljung, "Subspace-based multivariable system identification from frequency response data," IEEE Trans. Automat. Contr., vol. 41, no. 7, pp. 960-979, Jul. 1996.

[47] T. W. Anderson, An Introduction to Multivariate Statistical Analysis, 3rd ed. John Wiley \& Sons, 2003.

[48] A. M. Mathai, An Introduction to Geometrical Probability: Distributional Aspects with Applications. Newark, NJ: Gordon and Breach, 1999.

[49] A. Edelman, "Eigenvalues and condition numbers of random matrices," Ph.D. dissertation, MIT, Cambridge, MA, 1989.

[50] D. S. Baum and H. Bölcskei, "Impact of phase noise on MIMO channel measurement accuracy," in Proc. IEEE Veh. Technol. Conf. (VTC) Fall, vol. 3, Los Angeles, CA, Sep. 2004, pp. 1614-1618.

[51] M. K. Simon, Probability Distributions Involving Gaussian Random Variables: A Handbook for Engineers and Scientists. Kluwer Academic Publishers, 2002. 
[52] J. C. Hansen and H. Bölcskei, "A geometrical investigation of the rank-1 Ricean MIMO channel at high SNR," in Proc. IEEE Int. Symp. on Inform. Theory (ISIT), Chicago, IL, Jun. 2004, p. 64.

[53] G. W. Stewart, "The efficient generation of random orthogonal matrices with an application to condition estimators," SIAM J. Numerical Anal., vol. 17, no. 3, pp. 403-409, Jun. 1980.

[54] P. M. Lee, Bayesian Statistics: An Introduction, 2nd ed. London, UK: Arnold, New York, NY: Wiley, 1997.

[55] B. M. Hochwald, T. L. Marzetta, and V. Tarokh, "Multiple-antenna channel hardening and its implications for rate feedback and scheduling," IEEE Trans. Inform. Theory, vol. 50, no. 9, pp. 1893-1909, Sep. 2004.

[56] D. G. Michelson, V. Erceg, and L. J. Greenstein, "Modeling diversity reception over narrowband fixed wireless channels," in Proc. IEEE MTT-S Symp. on Technol. for Wireless Appl., Vancouver, BC, Canada, Feb. 1999, pp. 95-100.

[57] D. S. Baum, "Information-theoretic analysis of a class of MIMO channel measurement devices," Ph.D. dissertation, ETH Zurich, Zürich, Switzerland, 2007, No. 17395. 